\title{
A Generalized 2-D Multiport Model for Planar Circuits with Slots in Ground Plane
}

\author{
by
}

Amirreza Khajehnasiri

\begin{abstract}
A thesis
presented to the University of Waterloo

in fulfilment of the

thesis requirement for the degree of

Doctor of Philosophy

in

Electrical and Computer Engineering
\end{abstract}

Waterloo, Ontario, Canada, 2005

(c) Amirreza Khajehnasiri 2005 


\section{AUTHOR'S DECLARATION FOR ELECTRONIC SUBMISSION OF THE THESIS}

I hereby declare that I am the sole author of this thesis. This is a true copy of the thesis, including any required final revisions, as accepted by my examiners.

I understand that my thesis may be made electronically available to the public. 


\section{Abstract}

With increasing complexity of microwave integrated circuits and tendency towards building integrated modules, real estate in printed circuit boards becomes more at premium. On the other hand, building MIC's on a single semiconductor substrate such as GaAs has other drawbacks as substrate requirements for different components are sometimes contradictory. This has motivated researchers to consider multi-layer and stacked designs. Multi-layer planar circuits offer advantages that cannot be equalled by traditional single layer designs. In this respect, a new class of planar structures, based upon a multi-layered stack of dual-mode stripline or microstrip patches is becoming increasingly popular. In the new stacked design, coupling between planar circuits separated by a ground plane is accomplished through coupling apertures in the common ground plane.

This thesis is about developing an new approximate multiport network model for fast analysis of multi-layered planar structures with ground plane slots. To extend applicability of multiport network model (MNM) to the class of planar structures containing ground plane slots, a generalized network formulation for aperture problems is combined with traditional MNM to account for the presence of the slot. To this end, the slot is replaced by an unknown equivalent surface magnetic current. Slot ports are defined in terms of electric and magnetic fields over the slot in accordance with the generalized network formulation for aperture problems. While traditional MNM for planar circuits is based on generalized impedance matrices, we adopt a hybrid matrix approach for multi-layer structures. The hybrid matrix consists of four sub-matrices that relate terminal voltages and currents of edge and slot ports. The same generalized impedance matrix in the absence of the slot can be used to relate terminal voltages and currents of edge ports when the slot ports are short-circuited. Open circuit voltage at edge ports due to terminal voltages at slot ports and terminal currents at slot ports due to input currents at edge ports are represented by two transfer matrices. Both these transfer matrices can be calculated from 2D analysis which only considers $T M^{z}$ modes.

Interaction among slot ports, represented by a generalized admittance matrix, however, requires considering both $T M^{z}$ and $T E^{z}$ modes. This generalized admittance matrix is obtained from tangential component of the magnetic field over the slot due to the equivalent surface magnetic current and relates terminal voltages and currents of slot ports. Full modal expansion consisting of both $T M^{z}$ and $T E^{z}$ modes is used to compute the generalized admittance matrix of a slot in a regularly shaped planar cavity. For irregularly shaped patches, modal expansion is not avail-

able. Instead, a new contour integral equation for magnetic field, derived for the 
first time in this thesis, is combined with complex images method for calculation of generalized admittance matrix of a slot radiating in a planar cavity of arbitrary shape.

Once the hybrid matrix representation of a planar circuit on a ground plane containing a slot is derived, it can be connected to the hybrid matrix of any other planar circuit on the other side of the ground plane. This can be done by enforcing network equivalent of continuity of tangential fields across the slot. This leads to a generalized impedance matrix for the multi-layer structure relating terminal voltages and currents of edge ports of both planar circuits.

To show the accuracy of the proposed method of analysis, several proof-ofconcept structures have been analyzed by both this method and ANSOFT HFSS full-wave simulator as a reference. In most cases excellent agreement is achieved in predicting the return loss and radiation patterns of these multi-layer structures which proves the validity of the proposed approach for fast analysis and design of multi-layer planar structures. 


\section{Acknowledgments}

On academic level, I would like to thank my supervisor, professor Safieddin Safavi-Naeini for providing me with the opportunity to study at the University of Waterloo.

I would also like to extended my gratitude toward my defense committee members, professor Ke Wu, professor Sujeet Chaudhuri, professor Raafat Mansour, and professor James Martin for both accepting to be on the committee and reading my dissertation and also providing me with invaluable suggestions and comments for the future work. In particular, I am grateful to professor Ke Wu for traveling from Montreal to Waterloo to attend my defense and to professor James Martin for accepting to be on the committee on an extremely short notice.

I entered the EM world through a mistake, i.e. by entering the code for $\mathrm{RF} /$ Microwave discipline instead of Communication Systems, in which I was originally interested, in the nation-wide entrance examination for graduate studies in Iran. I had no intention of staying in this field if it was not for my M.Sc. supervisor, professor Rouzbeh Moini. I would like to thank him for being such a great mentor.

I am also deeply grateful to Wendy Boles and her staff in graduate studies office of Electrical and Computer Engineering department. Their patience, helpfulness and sympathy toward our problems is one of a kind.

This research was supported by grants from several companies and government agencies, which in alphabetical order are: CITO, DragonWave Inc., Metawave Communications Corporation, NSERC, and Research in Motion (RIM). I would like to thank each and every person involved in this process, in particular Mr. Donn Harvey and Mr. Shing Lee, my supervisors during my internships with Metawave Communications and DragonWave, respectively. 


\section{To MY PARENTS FOR THEIR UNWAVERING LOVE AND SUPPORT}




\section{Contents}

1 Introduction 1

1.1 Aperture-Coupled Microstrip Antennas . . . . . . . . . . . 3

1.2 Multi-Layered Planar Structures . . . . . . . . . . . . . . 6

1.3 Motivation for This Research . . . . . . . . . . . . . . . 10

1.4 Organization of the Thesis . . . . . . . . . . . . . . . . 12

2 Multiport Network Model 14

2.1 Introduction . . . . . . . . . . . . . . . . . 14

2.2 Basic Formulation of MNM . . . . . . . . . . . . . . 14

2.3 Derivation of Green's Function in terms of Eigenfunctions . . . . . . 20

2.4 Contour-Integral Equation . . . . . . . . . . . . . 23

2.4.1 Derivation of a Novel Contour-Integral Equation . . . . . . . 30

2.4.2 Discretization of Contour-Integral Equations . . . . . . . . . 32

3 Extension of 2D Multiport Network Model to ... 36

3.1 Generalized Network Formulation for Aperture Problems . . . . . . . . . . . . . . 36

3.1.1 Transmitted Power Considerations . . . . . . . . . . . . . . 40

3.2 Hybrid Matrix Formulation . . . . . . . . . . . . . . . . . . 41

3.3 Calculation of Impedance Matrix in terms of Hybrid Sub-Matrices . 45

3.4 Computation of Hybrid Sub-Matrices for

Regular Shapes . . . . . . . . . . . . . . . . . 46 
CONTENTS

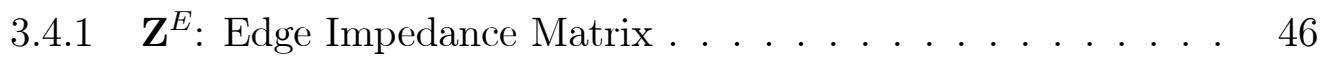

3.4.2 $\mathbf{H}^{E S}$ : Slot-To-Edge Transfer Matrix . . . . . . . . . . . 46

3.4.3 $\mathbf{Y}^{S}$ : Slot Admittance Matrix . . . . . . . . . . . . . . . . 48

3.5 Computation of Hybrid Sub-Matrices for

Irregular Shapes . . . . . . . . . . . . . . . . . . . . . . . . 49

3.5.1 $\mathbf{Z}^{E}$ : Edge Impedance Matrix . . . . . . . . . . . . . 50

3.5.2 $\mathbf{H}^{S E}$ : Edge-To-Slot Transfer Matrix . . . . . . . . . . 50

3.5.3 $\mathbf{H}^{E S}$ : Slot-to-Edge Transfer Matrix . . . . . . . . . . . 51

3.5.4 $\quad \mathbf{Y}^{S}$ : Slot Admittance Matrix . . . . . . . . . . . . . . . 52

4 Complex Images Method $\quad 55$

4.1 Introduction . . . . . . . . . . . . . . . . . . 55

4.2 Spectral Green's Functions for a Horizontal Electric and Magnetic Dipoles . . . . . . . . . . . . . . . . . . 57

4.2.1 Reflection and Transmission of Plane-Waves in Planarly Layered Media . . . . . . . . . . . . . . 58

4.2.2 Spatial Vector and Scalar Potentials for Multi-layered Media 60

4.3 Complex Images Method . . . . . . . . . . . . . . . . . . . . . . 65

4.3.1 Complex Images for an HMD in a Parallel-Plate Waveguide . . . . . . . . . . . . . . . 68

4.3.2 Numerical Results for Fields and Potentials of an HMD in a Parallel-Plate Waveguide Using Complex Images . . . . . . . 72

5 Numerical Results and Discussion $\quad 78$

5.0 .3 Single-pole two-layer filter . . . . . . . . . . . . . . 78

5.0 .4 Dual-Band Filter . . . . . . . . . . . . . . . . . . . . . . 81

5.0 .5 Multi-Mode Filter . . . . . . . . . . . . . . . . . . . 83

5.0 .6 Circular Patches _. . . . . . . . . . . . . . . 85

5.0.7 Corner-Cut Patches Coupled by an Inclined Slot . . . . . . . 87

5.1 Radiation Pattern . . . . . . . . . . . . . . . . . . . . . . . . 91 
CONTENTS

5.1.1 Reciprocity Approach to Pattern Computation of Microstrip Antennas . . . . . . . . . . . . . . . . . . 91

5.1.2 Radiation Pattern of an Integrated Antenna-Filter Device . 92

5.1.3 Radiation Pattern of Two Corner-Cut Patches Coupled by an Inclined Slot . . . . . . . . . . . . . . . . 94

6 Conclusion and Future Work $\quad 96$

6.1 Conclusion . . . . . . . . . . . . . . . . . . . 96

6.2 Future Work . . . . . . . . . . . . . . . . . . . . . . 98

$\begin{array}{ll}\text { A Proof of } 3.115 & 100\end{array}$

$\begin{array}{ll}\text { B Derivation of } 2.56 & 101\end{array}$

$\begin{array}{ll}\text { C Analytical Evaluation of... } & 103\end{array}$ 


\section{List of Figures}

1.1 Aperture-coupled microstrip antenna . . . . . . . . . . . . . 4

1.2 Stacked planar filters constructed from dual mode resonators . . . . 8

1.3 Multi-layered planar structure with microstrip resonators . . . . . 9

1.4 Examples of dual-mode planar filters . . . . . . . . . . . . 10

2.1 Generic planar circuit structure . . . . . . . . . . . . . . 15

2.2 Equivalence between co-planar feedline and vertical probe currents . 18

2.3 Concept of segmentation . . . . . . . . . . . . . . . . 22

2.4 Concept of desegmentation . . . . . . . . . . . . . . . 22

2.5 Region to which Green's theorem is applied. . . . . . . . . . . 25

2.6 Symbols used in contour-integral equation and numerical analysis . 30

3.1 General problem of aperture coupling . . . . . . . . . . 37

3.2 Generalized network interpretation of $3.90 \ldots \ldots \ldots$

3.3 An irregularly shaped patch on a ground plane with a slot . . . . 43

4.1 Reflection and transmission in a multilayered media . . . . . . . . 59

4.2 Three-layer structure backed by a ground plane . . . . . . . . . . 64

4.3 The integration paths of inverse Hankel transform . . . . . . . . 67

4.4 HMD in a homogeneous parallel-plate waveguide . . . . . . . . 68

4.5 Amplitude of $4 \pi \mu G_{q}$ from image expansion for $d=0.2 \lambda_{0}$ and $z=$

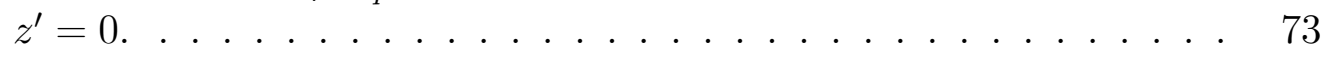

4.6 Amplitude of $4 \pi \mu G_{q}$ from modal expansion for $d=0.2 \lambda_{0}$ and $z=$

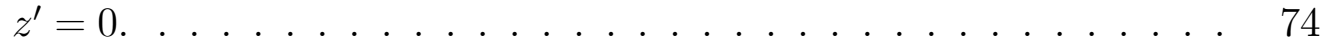




\section{LIST OF FIGURES}

4.7 Amplitude of $4 \pi \mu G_{q}$ from image and modal expansions as well as complex images for $d=0.2 \lambda_{0}$ and $z=z^{\prime}=0 \ldots \ldots \ldots . . \ldots 74$

4.8 Amplitude of $4 \pi \mu G_{q}$ from complex images method for $d=0.2 \lambda_{0}$ and $z=z^{\prime}=0$ : with $(-)$ and without $(--)$ fundamental mode extracted. 76

4.9 Amplitude of $4 \pi \mu G_{q}$ from image (-)and modal (--) expansions as well as complex images both with $(\Delta)$ and without $(x)$ extracting propagating modes for $d=0.2 \lambda_{0}$ and $z=z^{\prime}=0 \ldots \ldots \ldots$.

4.10 Amplitude of $H_{x}$ from image (-)and modal (--) expansions as well as complex images $(\Delta)$ for $d=0.2 \lambda_{0}$ and $z=z^{\prime}=0 . \ldots \ldots \ldots 77$

4.11 Amplitude of $H_{y}$ from image (-)and modal (--) expansions as well as complex images $(\Delta)$ for $d=0.2 \lambda_{0}$ and $z=z^{\prime}=0 . \ldots \ldots .77$

5.1 Two-layer single pole filter . . . . . . . . . . . . . . . . . 79

5.2 Return-loss of a single-pole two-layer filter . . . . . . . . . . 80

5.3 The amplitude of $S_{21}$ for the single-pole two-layer filter . . . . . 80

5.4 Two-layer dual-band filter . . . . . . . . . . . . . . . . . 81

5.5 Return-loss of two-layer dual-band filter . . . . . . . . . . . . 82

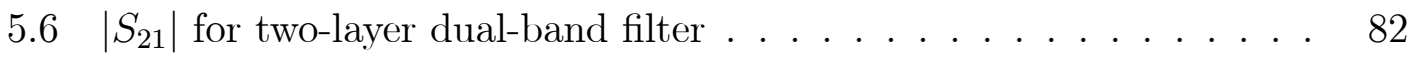

5.7 Multi-mode filter . . . . . . . . . . . . . . . 83

5.8 Return-loss for multi-mode filter . . . . . . . . . . . . . . . 84

$5.9\left|S_{21}\right|$ for multi-mode filter $\ldots \ldots \ldots \ldots \ldots \ldots$

5.10 Two circular patches coupled through a slot in ground plane . . . . 85

$5.11\left|S_{11}\right|$ for Two circular patches coupled through a slot in ground plane 86

$5.12\left|S_{21}\right|$ for Two circular patches coupled through a slot in ground plane 86

5.13 Corner-cut patches coupled through an inclined slot . . . . . . . . 88

$5.14\left|S_{11}\right|$ for corner-cut patches coupled through an inclined slot $\ldots .88$

$5.15\left|S_{21}\right|$ for corner-cut patches coupled through an inclined slot $\ldots . \quad 89$

5.16 Normalized radiated power from structure shown in Fig. 5.13 . . . 89

$5.17\left|S_{11}\right|$ for the shielded structure in Fig.. $5.13 \ldots \ldots \ldots$

5.18 Dipole in the far-field of a microstrip antenna $\ldots \ldots \ldots$. . . . 91

$5.19\left|E_{\theta}\right|$ for the structure in Fig. 5.1 at $\varphi=45^{\circ} \ldots \ldots \ldots$ 


\section{LIST OF FIGURES}

$5.20\left|E_{\varphi}\right|$ for the structure in Fig. 5.1 at $\varphi=45^{\circ} \ldots \ldots \ldots$

$5.21\left|E_{\theta}\right|$ for the structure in Fig. 5.13 at $\varphi=45^{\circ} \ldots \ldots \ldots$

$5.22\left|E_{\varphi}\right|$ for the structure in Fig. 5.13 at $\varphi=45^{\circ} \ldots \ldots \ldots \ldots$

B.1 Symbols used in the derivation of the contour-integral equation. . . 102 


\section{List of Tables}

4.1 Potential functions for different kinds of sources . . . . . . . . . 57 


\section{Chapter 1}

\section{Introduction}

Multi-layer circuit technology offers many advantages, including reliability, possibility of mass production, and reduction in size and cost. This technology has even extended to higher frequencies, well into the microwave range, replacing rigid and bulky waveguides at low power levels by low-profile planar structures and culminating with the advent of Microwave Integrated Circuits (MIC's) and Monolithic MIC's (MMIC's). Commonly used planar structures in microwave frequencies includes microstrip lines, tri-plate striplines, coplanar lines, and slot lines. Among all planar structures, however, microstrip line is the most popular and the most widely used structure and has received considerable attention in many respects.

Due to the inhomogeneous nature of the dielectric in microstrip lines, an accurate analysis of these structures is much more complicated than the analysis of traditionally used transmission lines and waveguides. Numerous authors and researchers have addressed analysis and design issues of planar structures in the past three decades and thanks to their efforts, today a rather extensive arsenal of analysis and simulation tools exists for microstrip lines and structures.

The complexity of existing numerical methods, such as Method of Moments (MoM), Finite-Elements Method (FEM), and Finite-Difference Time-Domain Method (FDTD), etc., makes them unsuitable for design optimization of planar circuits and necessitates use of fast methods specifically developed for planar structure, where reasonable approximations are made to simplify the problem without compromising the accuracy of solutions.

It is clear that microstrip lines (and most other planar structures except for the tri-plate striplines) cannot support pure transverse electromagnetic (TEM) modes. This is because of the fact that the electromagnetic fields within the structures 


\section{CHAPTER 1. INTRODUCTION}

are not bound to the dielectric substrate and they extend across the interface into the surrounding medium, a fact that has led to the development of low-profile microstrip antennas. Since TEM waves propagate with intrinsic phase velocity of the dielectric medium and because of the fact that the phase velocities are different in the two media, it is almost evident that these structures are not capable of supporting pure TEM modes. In other words, in addition to transverse electric and magnetic fields, longitudinal components must also exist (hybrid modes). However, at low frequencies these longitudinal components are negligible compared to the transverse components and a quasi-TEM analysis is possible.

As the frequency increases, fields become more confined within the dielectric substrate. Since phase matching requires the electromagnetic waves to propagate with the same velocity within the dielectric and surrounding medium, they can no longer propagate at the intrinsic phase velocity of either media, hence producing dispersion. Therefore, at higher frequencies quasi-TEM approximation becomes inadequate.

At frequencies where the quasi-TEM approximation is not valid anymore, fullwave analysis methods, such as MoM, FEM, and FDTD can be used to accurately predict the behavior of planar structures. In most cases, however, we are not interested in a full-wave analysis of the planar structure, which can be quite timeconsuming. This is specially true in integrated microstrip circuits consisting of several patches coupled or connected (directly or indirectly) with each other and probably with active components to perform a specific task. For example, several dual-mode patches can be gap-coupled to build a Chebyshev or elliptical filter. In these cases, we are only interested in terminal properties of microstrip structures as if they are lumped circuit elements. Multi-port Network Model for planar circuits is one of the methods that addresses this issue. Multi-port Network Modeling (MNM), which has been developed based on low-frequency circuit theory, builds an analogy between the planar structure and a low-frequency lumped-element network. Each planar structure is characterized by a generalized impedance matrix, which can be connected to the impedance matrices of other components (distributed or lumped, passive or active) to characterize the whole circuit by its low-frequency terminal variables. But MNM has its own limitations, due to the fact that it has been specifically developed for thin planar structures.

As the complexity of microstrip circuits increases, real estate becomes more at premium. The straightforward approach of building an MIC on a single semiconductor substrate such as GaAs has several drawbacks. There is generally not enough space on a single layer to hold all components. For example, consider an integrated

millimeter wave array or sub-array. In a single layer design, the semiconductor 


\section{CHAPTER 1. INTRODUCTION}

substrate must hold antenna elements, active phase-shifter, amplifier circuitry, bias lines, and RF feed lines. More importantly, the high permittivity of a semiconductor substrate such as GaAs has a destructive effect on bandwidth performance of the antenna element, which is best for low dielectric constant substrates. To some degree, this can be compensated for by increasing the thickness of the substrate, which in turn increases spurious feed radiation and power launched into surface waves and eventually leads to scan blindness [1]. In recent years, these problems have motivated some researchers to consider multi-layer and stacked designs. Multilayer planar circuits offer advantages that cannot be matched by traditional single layer designs. As the popularity of multi-layer and stacked designs increases, the necessity of fast analysis and design methods becomes more evident. MNM which has been successfully employed in single-layer designs is useless for multi-layer structures. It is this lack of fast analysis methods that has motivated us to extend the applicability of MNM into multi-layer planar structures.

In order to better introduce the class of multi-layer structures that we will be dealing with and also portray a better picture of their advantages, we start with aperture-coupled microstrip antenna [2], which has been successfully used in many applications.

\subsection{Aperture-Coupled Microstrip Antennas}

Microstrip antenna technology came into the spotlight in the late 1970s. The development of this new technology was so rapid that by the early 1980s there already was fairly well established methods for analysis, design and modeling of basic microstrip antenna elements and arrays. At the same time, it was becoming obvious that the performance of basic microstrip antenna elements fed by coaxial cables or coplanar feeds could not be improved beyond a certain limit. The designers simply did not have many parameters to play with in order to improve the performance. By researchers turning their attention to improving antenna performance features such as bandwidth, new feeding structures that would provide more degrees of freedom seemed unavoidable. In addition, advances in PCB technology and looming importance of integrated circuit applications such as integrated phased array systems using microstrip antennas, that would perfectly suit low-cost and high-density integration with active MIC or MMIC phase-shifters and RF circuitry, were among other driving forces behind this effort. One of the most intriguing and most successful feed systems, is aperture-coupled microstrip antenna (Fig. 1.1) developed by Pozar [2], [3]. 


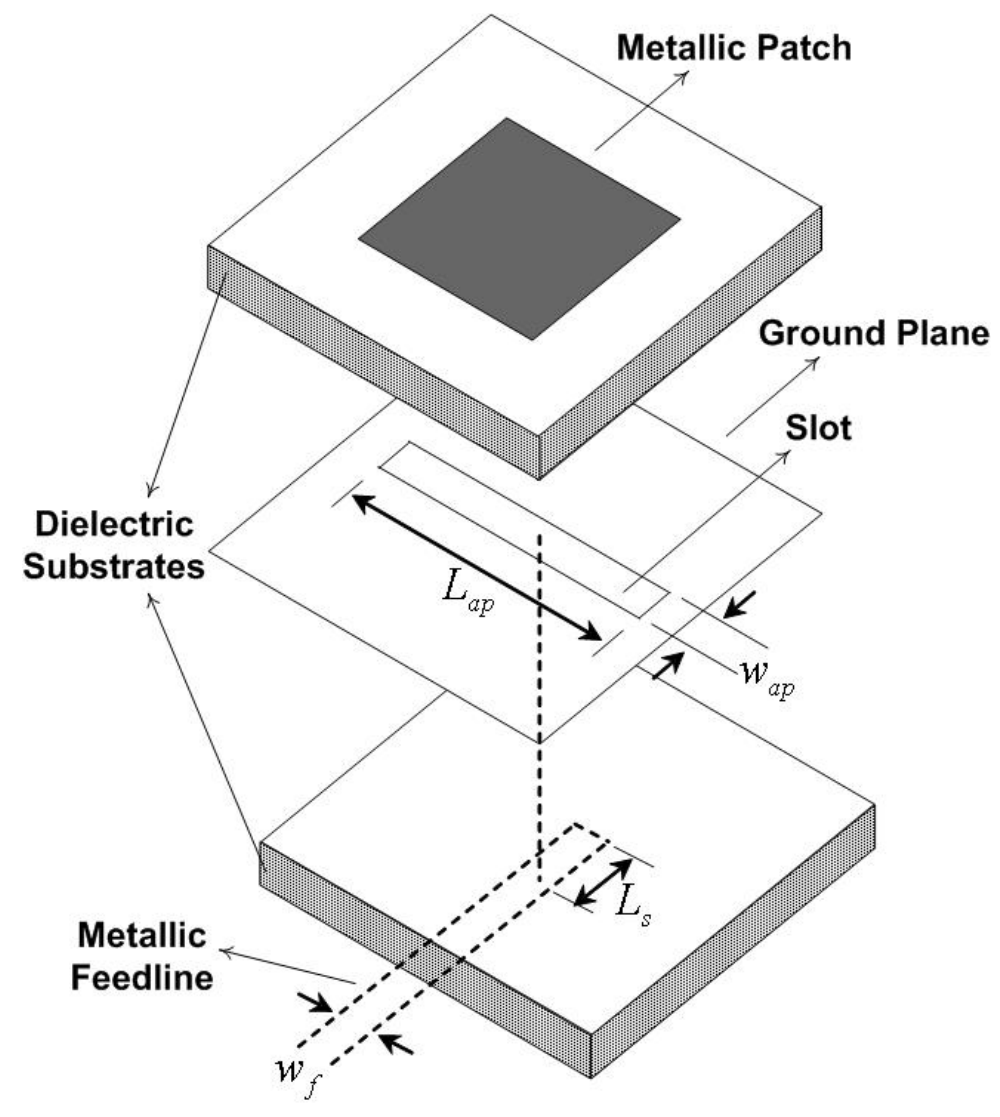

Figure 1.1: Aperture-coupled microstrip antenna

The idea is to use two different substrates on two sides of a common ground plane. One substrate holds the feed circuitry and the other supports a resonant microstrip patch. The feed circuitry and the microstrip element are coupled through a slot or an aperture in the ground plane. The first prototype element was built using a circular hole and was analyzed by small hole coupling theory ([2]). Even though small hole coupling theory was only suitable for small apertures, the analysis results for the prototype element suggested that dominant coupling mechanism was magnetic and would be maximized for narrow rectangular slots centered underneath the slot. These were later confirmed by more accurate numerical modeling [4], [5]. With two-layer design, the engineers are at liberty to chose among almost a dozen material and dimensional parameters, such as:

1. Antenna substrate dielectric constant to adjust the bandwidth and radiation 


\section{CHAPTER 1. INTRODUCTION}

efficiency of the antenna. Lower dielectric constant materials yield wider impedance bandwidth and reduced surface wave excitation.

2. Antenna substrate thickness which affects bandwidth and coupling level. Wider bandwidth is achieved by employing thicker substrates. However, for a given aperture size, the coupling is reduced as the substrate thickness increases.

3. In fundamental resonance mode of operation, the length of the patch radiator determines the resonant frequency of the antenna, while the microstrip patch width affects the resonant resistance of the antenna, with a wider patch giving a lower resistance.

4. Feed substrate dielectric constant can be selected independently for good microstrip circuit qualities, typically in the range of 2 to 10

5. Feed substrate can be chosen thinner for less spurious radiation from feed lines

6. Slot length affects the coupling level and also the back radiation level. So does slot width, but to a lesser degree. Slots with enlarged ends, such as dog-bone, bow-tie, or $\mathrm{H}$-shaped apertures can further improve coupling.

7. Feed line position and orientation relative to the slot affects coupling level, so does the position of the patch relative to the slot.

8. Length of the tuning stub can be used to tune the excess reactance of the aperture coupled antenna.

The practical implications of abundance of design parameters are invaluable. Each parameter can be used to adjust a particular performance benchmark without substantially affecting the others. For example, dual polarization can be obtained by using two orthogonal feeds and two orthogonal slots [6], [7], [8], [9] without being concerned about real estate (especially in phased arrays) and spurious radiation from feed. Circular polarization can be achieved by a single diagonal slot and a slightly non-square patch [10] or by using a crossed slot with a single microstrip feedline through the diagonal of the cross and a slightly non-square patch [11].

Aperture coupled microstrip antennas are very suitable for arrays using either series [12] or corporate feed networks [13], [14], [15], especially in dual-polarized or dual frequency arrays. The two-layer structure makes plenty of space available for feed network, whereas the ground plane effectively shields the radiating element 


\section{CHAPTER 1. INTRODUCTION}

and the feed circuitry. A ground plane located some distance below the feed layer can be used to eliminate radiation in the back direction.

In general, some of the useful features that can be achieved by aperture coupled feed for microstrip antennas can be summarized as:

- Impedance bandwidths ranging from $10 \%$ to $15 \%$ with a single layer antenna [6], [16] (limited to only 2-5\% with traditional feeds), and up to $30-50 \%$ with stacked patch configuration [13], [17], [18], [19] mainly due to additional degrees of freedom offered by the stub length and coupling aperture size.

- Independence in selection of antenna and feed substrate materials

- Shielding between the radiating aperture and the feed network due to twolayer construction

- Increased substrate space for antenna elements and feed lines

- Convenient integration for active arrays

- Theoretically zero cross polarization in principle planes

- Many parameters for performance adjustment, such as patch shape, aperture shape, feed line type, etc.

Most of these features carry over to other two-layer designs such as integrated antenna-filter devices or multi-layer filters.

\section{$1.2 \quad$ Multi-Layered Planar Structures}

In many applications weight, cost, and size are amongst the determining factors for component selection. For example, dual-mode cavity and dielectric resonator filters have become the mainstay of satellite communications, replacing their bulky single-mode predecessors. In addition to drastic reduction in weight, size, and cost, dual-mode filters have also made possible realization of filtering functions such as elliptic function filters which require cross-coupling between non-adjacent cavities to produce alternate forward paths from input to output. In the past decade, dual-mode cavity and dielectric resonator filters have been gradually replaced by 


\section{CHAPTER 1. INTRODUCTION}

even significantly less bulky, cheaper and lighter dual-mode planar filters [20] in lowpower applications. The advent of new high temperature superconductive materials and advancements in fabrication technology has contributed to this development.

In communication systems, however, where filters and other components such as antennas, phase-shifters, and active components are connected to perform a specific task, it is the physical dimensions and performance of the system front-end that matters the most. For example, when the receiving antenna is followed by a bandpass filter, it is desirable to integrate the antenna and the bandpass filter in a single module. However, juxtaposition of the antenna element and the bandpass filter on the same layer is not practical. The presence of the distributed filter can adversely affect the performance of the antenna element. This is especially true in arrays, where the area allocated to each radiating element is barely larger than the size of each element. Similar to the aperture-coupled microstrip antennas, multi-layered structures can accommodate both radiating elements and filter components without compromising their performance. In addition, integration of antenna/filter devices can improve bandwidth performance of the antenna (It has been demonstrated that the bandwidth performance of resonant antennas can be enhanced using impedance matching techniques without altering its radiating characteristics [21], [22]).

The new class of planar structures, based upon a multi-layered stack of dualmode stripline or microstrip patches, was introduced by Curtis and Fiedziuszko [23]. In the new stacked design, coupling between the dual-mode patches separated by a ground plane is accomplished through coupling apertures in the common ground plane. The coupling mechanism is very much the same as in aperture-coupled microstrip antennas. The multi-layered design has the advantage over the singlelayer design in that it offers more degrees of freedom for the designer to choose from, such as the dielectric constants and thicknesses of each substrate, size and shape of the coupling aperture and size and shape of each metallic patch.

It is important to note that we are only interested in multi-layered structures where layers are separated by ground planes containing slots. There are other designs which are referred to as multi-layer structures by their respective authors, but do not fit in this category. For example, Le Nadan et al. demonstrate integration of a unipolar slotline dipole [24] and a square coplanar waveguide fed patch antenna [25] with a bandpass filter in an integrated antenna/filter device. In this approach, the resonant antenna is treated as a lossy resonator in the filter network. A variation of the design in [25], where the bandpass filter is completely implemented on a single layer and does not involve any via holes has been reported in [26]. Waterhouse et al. use annular ring to suppress the $T M_{0}$ surface wave mode in a probe fed microstrip antenna with superstrate, over which the annular 


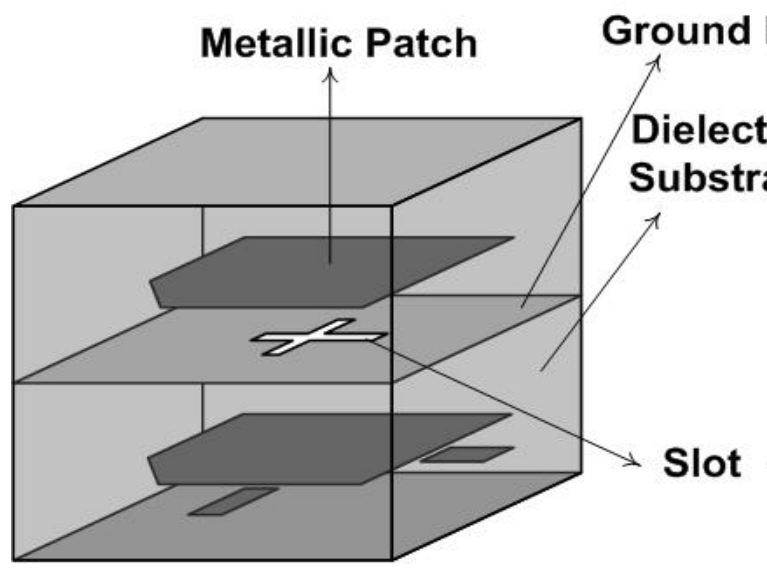

(a)
Ground Plane

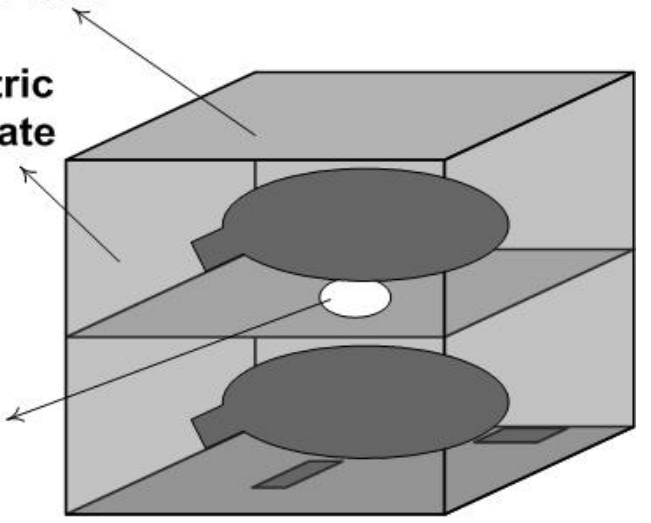

(b)

Figure 1.2: Stacked planar filters constructed from a) square b) circular dual mode resonators [23]

ring is etched [27] and term it as a multi-layer design. In the terminology used in this thesis, these structures do not qualify as multi-layer designs. Nonetheless, the aforementioned designs can be combined with stripline and microstrip layers in a stacked configuration for enhanced performance.

The stripline based structures proposed in [23] consist of rectangular or circular dual mode stripline resonators (Fig. 1.2). In case of square resonators, the two dual-mode patches are coupled through a cross-shaped slot in the ground plane. For circular resonators, the coupling aperture is also circular. In both cases, each resonant mode is directly coupled only to one mode of the other resonator, which means that each resonator is coupled to more than one resonator, hence implementation of a 4-pole filter with transmission zeros is feasible. As a proof of concept design, implementation of a 4-pole elliptic function filter with the structure in Fig. $1.2 \mathrm{a}$ is reported in [23].

Based on a similar approach, a two-layer filter consisting of microstrip resonators (Fig. 1.3) has also been proposed [23]. Though the structure in Fig. 1.3 cannot be extended to more than two layers solely by using microstrip patches, it can be combined with stripline based structures for multi-layer designs. In addition, this two-layer structure is the starting point in development of our proposed approach which will be introduced in following chapters.

Multi-layer structures consisting of dual-mode planar resonators coupled through 


\section{CHAPTER 1. INTRODUCTION}

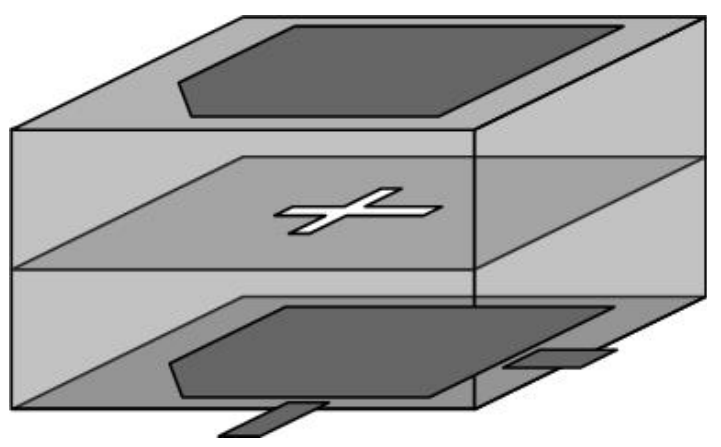

Figure 1.3: Multi-layered planar structure with microstrip resonators

apertures in ground plane can result in significant reduction in size and better use of the real estate on the chip. For example consider the structure in Fig. 1.4a. In the absence of any perturbations, each of the square resonators can support two orthogonal modes, which are theoretically isolated and energy from one mode cannot be coupled to the other one. By introducing the small triangular cutout, the energy can be coupled from one mode to the other. The amplitudes of these degenerate modes are controlled by the size of the triangular cutout [28]. Therefore, for each dual-mode patch, we have two coupled resonators.

To realize dual-mode planar filters of orders $n>2$, dual-mode resonators are arranged in a planar manner with physically adjacent resonators capacitively coupled, either directly or through a short piece of planar transmission line, as shown in Fig. 1.4a and Fig. 1.4b. While there is only two physical resonators, the structure shown in Fig. 1.4a can be used to realize a 4-pole Chebyshev filter [20]. Similarly, the structure shown in Fig. 1.4c can be used to implement an 8-pole elliptic function filter [20]. As it was discussed before, however, spreading out distributed circuits horizontally on a single layer has some drawbacks. An alternative approach is to arrange dual-mode resonators both horizontally and vertically. For example the same 8-pole elliptic function filter can be realized by aperture coupling the 4-pole planar filter in Fig. 1.4a (or Fig. 1.4b) with a similar structure on the other side of the ground plane.

Development of a new method of analysis for this class of multi-layered planar structures consisting of microstrip resonators is the focus of this thesis. These structures can be used either as multi-layer filters or integrated antenna/filter devices. It is important to note that the applicability of this new method of analysis is not limited to slot-coupled dual-mode square or circular resonators. As a matter of fact, we do not impose any restrictions on the shape of the planar circuits or the 
CHAPTER 1. INTRODUCTION

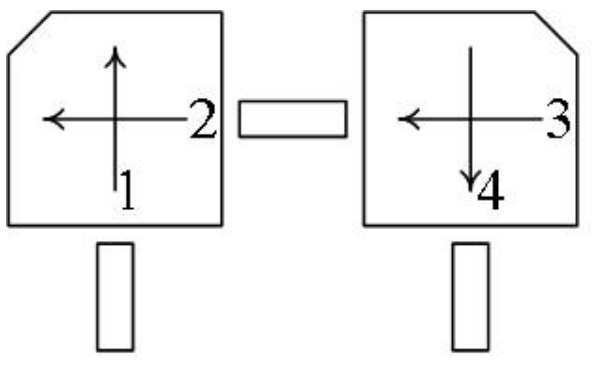

(a)

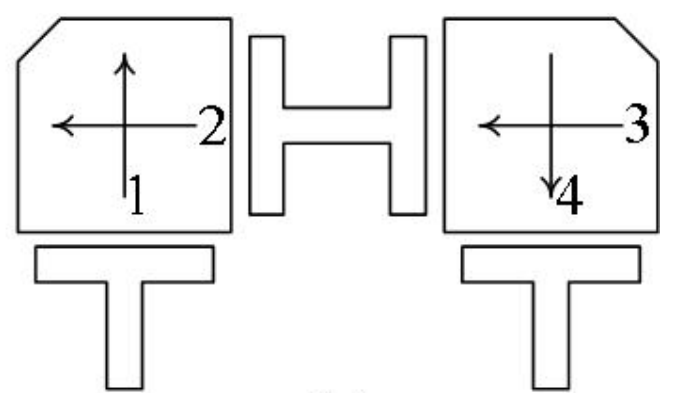

(b)

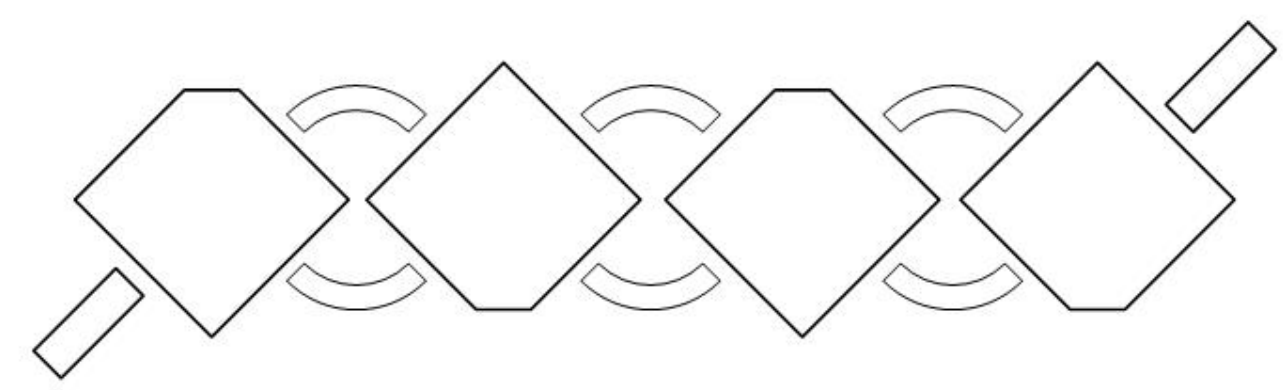

(c)

Figure 1.4: Examples of dual-mode planar filters: a) 4-pole Chebyshev filter [20], b) 4-pole Chebyshev filter with alternate coupling method [29], c) 8-pole elliptic function filter [20]

coupling aperture.

\subsection{Motivation for This Research}

The presence of two dielectric layers and the interaction between resonators through the slot complicates the analysis of multi-layer planar structures. Commercially available software based on full-wave analysis methods such as finite elements method, while very rigorous, are not suitable for design and design optimization purposes of these structures. In fact, due to the rapid changes of fields in close vicinity of the slot, the design process can be very time-consuming. Approximate numerical methods developed specifically for the analysis of planar structures, to 


\section{CHAPTER 1. INTRODUCTION}

some extent, can solve these problems. In particular, Multi-port Network Modeling of planar structures in association with segmentation and desegmentation methods of analysis is very suitable for the analysis and design of microstrip or stripline structures with arbitrary shapes, such as dual-mode resonators. For planar configurations (single-layer structures), coupling between resonators can be taken into account by Edge Admittance Networks [30]. In addition, coupling between resonators on a single-layer is a rather well understood topic and accurate models exist which can be used in conjunction with MNM for design purposes. However, the multi-port network modeling approach is not applicable to multi-layer structures. To the best our knowledge, there does not exist any MNM based method for fast analysis and design optimization purposes of multi-layer planar structures, though some authors have addressed the issue in some very special cases, mainly very narrow or small aperture-coupling of a microstrip line to a patch antenna.

A network modeling for a microstrip line coupled to a microstrip antenna through a small circular aperture in the ground plane was first introduced by Gao and Chang [31]. Their approach is based on aperture coupling theory and quasi-TEM analysis of the microstrip line by conformal mapping method. They eventually derive a six port equivalent network of the aperture-coupled microstrip antenna, from which scattering matrix of the structure can be derived. This method of analysis can only be applied to rectangular patches and circular apertures, as it has been based on transmission line analysis of the patch and small-aperture coupling theory. It is also important to note that despite the similarity in name, this method is substantially different from ours.

Himdi et al. offer a cavity based model for input impedance variations of an aperture-coupled microstrip antenna [32], [33], [34], [35]. In this approach, an equivalent magnetic current is used to replace the slot. However, in order to simplify the analysis they assume that the magnetic current is uniformly distributed over the slot. Then the microstrip antenna is modeled as a two-dimensional cavity supporting only the dominant $T M_{10}$ mode. To the input microstrip line, the slot looks as a shunt susceptance, which is modeled as the admittance of two shortcircuited slotlines. The problem with this approach is that it ignores the threedimensional behavior of the slot inside the cavity, in other words oversimplifies the problem. On the other hand, it is only applicable if the patch and the slot are both rectangular.

CAD software for analysis, design, and optimization of low-frequency circuits and networks has reached a fairly high level of refinement. With the aid of multiport network modeling, many of these tools can be equally well used for analysis and design of planar structures. This has motivated us to extend applicability of 


\section{CHAPTER 1. INTRODUCTION}

multi-port network modeling to multi-layer structures. The structure which will be the focus of this thesis is shown in Fig. 1.3, with the difference that we do not impose any restrictions on the shape or size of the patches and the slot. We start by traditional multi-port network modeling of planar circuits and combine it with the generalized network formulation for aperture problems to arrive at an equivalent network representation for a metallic patch of an arbitrary shape etched on a grounded dielectric slab, with the ground plane containing a slot of an arbitrary shape. Once such a network equivalence for the planar circuit on a groundplane containing a slot is established, the equivalent networks of planar circuits on opposite sides of a ground plane can be connected according to conventional MNM to derive an equivalent network for the whole structure. The following section elaborates on the organization of this thesis.

\subsection{Organization of the Thesis}

In order to introduce the new method for analysis and design of multi-layered planar circuits we follow a systematic approach for presentation. We start with an introduction to the traditional multi-port network model for single-layer planar circuits. Our presentation mainly follows that of Okoshi [36] as far as the basic formulation of MNM is concerned. However, we follow a different path for derivation of contour integral equation which is more general than Weber's solution for cylindrical waves. We also derive a novel contour integral equation for the magnetic field, which, to the best our knowledge, has not been used before. We also show that the discretized form of the new contour integral equation can be used as the inverse of the traditional contour integral equation, obviating the need for additional matrix inversion in cases the generalized admittance matrix of a single-layer planar circuit is required.

Chapter 2 focuses on extension of MNM to planar circuits with ground plane slots. First the generalized network formulation in which an aperture problem is characterized by a generalized admittance matrix is introduced. Then we continue by presenting hybrid matrix formulation for planar circuits containing ground plane slots and calculation of impedance matrix in terms of hybrid sub-matrices. We present two implementation of extended MNM for multi-layer planar circuits, one for regular shapes and one for irregular (arbitrary) shapes. The implementation for regular shapes is based on eigenfunction expansion of impedance Green's function. 


\section{CHAPTER 1. INTRODUCTION}

For irregular shapes, we utilize both traditional and newly derived contour integral equations.

In the third chapter we introduce complex images method which has been used in calculation of tangential magnetic fields due to the equivalent surface magnetic current. We start the chapter by deriving spectral Green's functions for both a horizontal electric and horizontal magnetic dipoles in a planarly layered media in terms of Fresnel's reflection coefficients. Spatial vector and scalar potentials for multi-layered media and a formal presentation of the complex images methods will follow this section. Then we apply complex images method to a horizontal magnetic dipole in a homogenous parallel-plate waveguide. We conclude this chapter by presenting results from complex images method for an HMD in a parallel-plate waveguide and comparing them to the results from image and modal expansions.

Chapter 5 is dedicated to numerical results. To show accuracy of our proposed method we have analyzed a few structures both with our method and with ANSOFT HFSS full-wave simulator as a reference. In this chapter, we will also present a method based on reciprocity for calculation of radiation pattern of microstrip antennas in the far-field.

Chapter 6 gives a summary of the thesis and outlines the future work related to this research. 


\section{Chapter 2}

\section{Multiport Network Model}

\section{$2.1 \quad$ Introduction}

Originally developed for analysis and design of tri-plate stripline structures, Multiport Network Modeling (MNM) has evolved considerably in the course of the years and has been successfully applied to other types of planar structures, including antennas, arrays, filters, microwave integrated circuits, etc. MNM is based on the assumption that thin planar circuits are essentially two-dimensional electromagnetic environments, with field variation only in transverse direction.

\subsection{Basic Formulation of MNM}

An irregularly shaped metallic patch etched on a linear, homogeneous, and isotropic dielectric slab backed by a ground plane, Fig. 2.1, constitutes a generic microstrip structure. Depending on the dielectric constant and thickness of the dielectric slab and the shape of the patch, the aforementioned structure can be a resonator or an antenna.

As can be seen from Fig. 2.1, the coordinate system is chosen in a way that the metallic patch lies in $z=d$ plane and the ground plane coincides with $x y$ plane. Unless there is a coupling port, the periphery of the circuit is assumed to be covered by PMC (perfect magnetic conductor) walls. This is one of the fundamental 


\section{CHAPTER 2. MULTIPORT NETWORK MODEL}

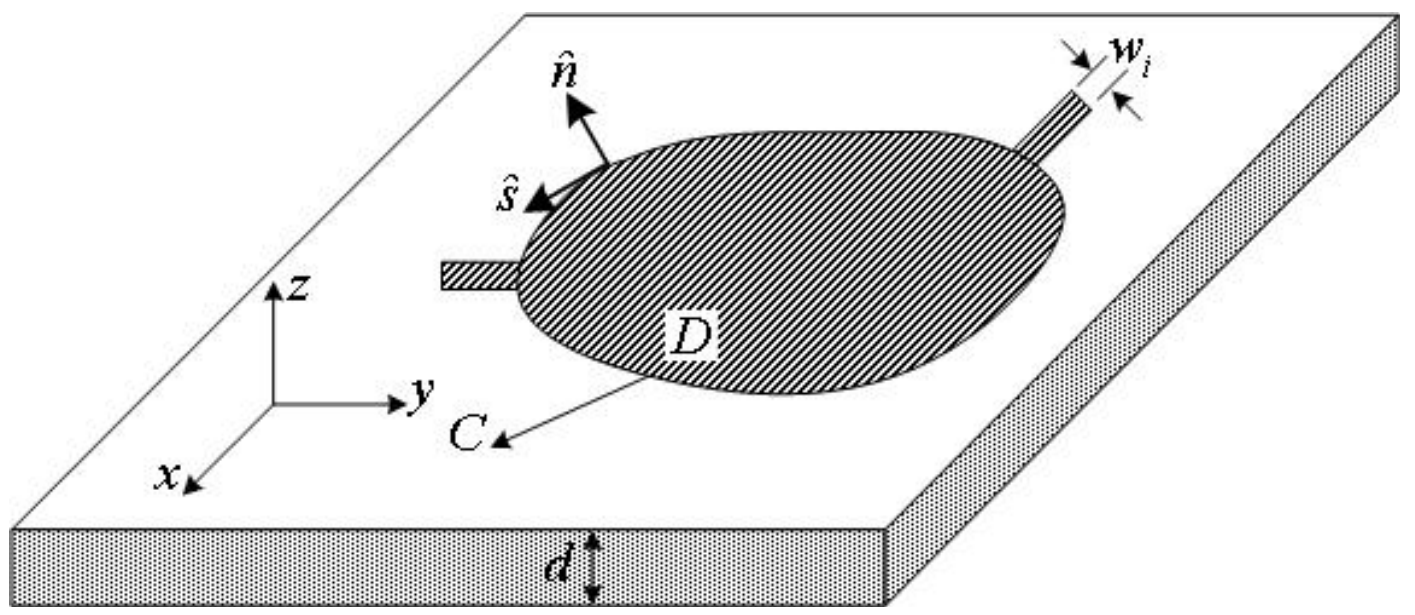

Figure 2.1: Generic planar circuit structure

assumptions in MNM of thin planar circuits and will be justified shortly. If the thickness of the dielectric slab $d$ is much smaller than the wavelength in dielectric medium, one can assume that there is no field variation in $z$ direction, i.e. $\partial / \partial z=0$. Consequently, $E_{x}$ and $E_{y}$ have to be identically zero, as they vanish both on the patch and the ground plane and do not vary in $z$ direction. This also implies that $H_{z}=0$. Hence, the only nonzero components are $E_{z}, H_{x}$, and $H_{y}$, which are related by Maxwell's equations:

$$
\begin{aligned}
j \omega \varepsilon E_{z} & =\frac{\partial H_{y}}{\partial x}-\frac{\partial H_{x}}{\partial y} \\
-j \omega \mu H_{x} & =\frac{\partial E_{z}}{\partial y} \\
j \omega \mu H_{y} & =\frac{\partial E_{z}}{\partial x}
\end{aligned}
$$

where $\omega, \varepsilon$, and $\mu$ represent the radian frequency of operation, permittivity and permeability of the dielectric medium, respectively. Equations 2.1a to 2.1c in connection with the relevant boundary conditions are sufficient to characterize any two-dimensional planar circuit. $H_{x}$ and $H_{y}$ can be eliminated from 2.1 a to give the two-dimensional Helmholtz equation for the planar structure in terms of $E_{z}$ :

$$
\left(\nabla_{t}^{2}+k^{2}\right) E_{z}=0
$$

where $\nabla_{t}^{2}$ and $k=\omega \sqrt{\mu \varepsilon}$ are the transverse Laplacian operator and wavenumber 


\section{CHAPTER 2. MULTIPORT NETWORK MODEL}

in the dielectric medium, respectively and we have:

$$
\nabla_{t}^{2}=\frac{\partial^{2}}{\partial x^{2}}+\frac{\partial^{2}}{\partial y^{2}}
$$

Evidently, 2D planar circuits only support $T M^{z}$ modes. Since there is no transverse electric field components, a unique voltage can be defined between the patch and the ground plane as $V=d E_{z}$. In addition, the surface current density on the patch, denoted by $\vec{J}_{\text {sur }}$, is related to the magnetic field by:

$$
\vec{J}_{\text {sur }}=\hat{z} \times \vec{H}=\hat{y} H_{x}-\hat{x} H_{y}
$$

Upon combining 2.4 with 2.1 b and $2.1 \mathrm{c}$, we obtain:

$$
\hat{x} \frac{\partial E_{z}}{\partial x}+\hat{y} \frac{\partial E_{z}}{\partial y}=\nabla E_{z}=-j \omega \mu \vec{J}_{\text {sur }}
$$

Let $\hat{n}$ denote the outward normal to the periphery of the circuit at some point. At that point we will have:

$$
\frac{\partial E_{z}}{\partial n}=\hat{n} \cdot \nabla E_{z}=-j \omega \mu\left(\hat{n} \cdot \vec{J}_{\text {surf }}\right)
$$

On the periphery $\hat{n} \cdot \vec{J}_{\text {sur }}$ is in fact the surface current flowing out of the circuit. If no external port is connected to the circuit at that point and if one assumes that no current flows between the upper and lower sides of the patch over the periphery of the metallic patch, we will have $\hat{n} \cdot \vec{J}_{\text {sur }}=0$. Hence:

$$
\frac{\partial V}{\partial n}=\frac{\partial E_{z}}{\partial n}=0
$$

This is the boundary condition that has to be satisfied on the boundary of the planar circuit. From

$$
\hat{s} \cdot \vec{H}=-\frac{1}{j \omega \mu} \frac{\partial E_{z}}{\partial n}=0
$$

where $\hat{s}=\hat{n} \times \hat{z}$ is the tangent to the boundary of the planar circuit (Fig. 2.1). Equation 2.8 proves that for thin planar structures the tangential magnetic field vanishes on the boundary. In other words, the periphery of the circuit is covered by $\mathrm{PMC}$ walls. If an external port is connected to the circuit, the boundary condition becomes:

$$
\frac{\partial V}{\partial n}=d \frac{\partial E_{z}}{\partial n}=j \omega \mu d J^{E}
$$




\section{CHAPTER 2. MULTIPORT NETWORK MODEL}

where $J^{E}$ is the input current flowing into the circuit from an external port. Hereinafter, the superscript $E$ will be used to denote variables and quantities associated with the edge of the metallic patch. In order to build an analogy between field and circuit variables, the periphery of the planar circuit, the contour $C$ as shown in the Fig. 2.1, is divided into sufficient number of smaller segments and each segment is treated as a port in low- frequency circuits having a terminal voltage and current defined as:

$$
\bar{V}_{j}^{E} \equiv \frac{d}{w_{j}} \int_{w_{j}} E_{z}(s) d s \quad, \quad \bar{I}_{j}^{E} \equiv \int_{w_{j}} J^{E}(s) d s
$$

where $s$ is the distance measured along the contour $C$ in a counterclockwise direction and the integration is performed over the width of pertinent port, i.e. $w_{j} . \bar{V}_{j}^{E}$ and $\bar{I}_{j}^{E}$ are the terminal voltage and terminal current of the $j^{t h}$ port. If there are $N^{E}$ segments along the edge of the circuit, the network equivalent of the planar structure will consist of $N^{E}$ ports. Such a network can be uniquely identified by an $N^{E} \times N^{E}$ impedance matrix relating voltage of each port to its current and those of all other ports. This impedance matrix, hereinafter, will be referred to as the Edge Impedance Matrix and will be denoted by $\mathbf{Z}^{E}$. In order to derive $\mathbf{Z}^{E}$ for a planar circuit, an impedance Green's function is needed to relate $\bar{J}_{j}^{E}$ 's and $\bar{V}_{j}^{E}$ 's. In reality such an impedance Green's function can be quite complicated. However, for a $z$-directed electric current source $J_{z}\left(x_{0}, y_{0}\right)$, such as the axial current through a $z$-directed coaxial probe, the impedance Green's function, which relates $V(x, y)$, the induced voltage at $(x, y)$, to $J_{z}\left(x_{0}, y_{0}\right)$, is rather simple. This Green's function denoted by $\mathcal{G}\left(x, y \mid x_{0}, y_{0}\right)$ can be defined as [37], [38]:

$$
V(x, y)=\iint_{D} \mathcal{G}\left(x, y \mid x_{0}, y_{0}\right) J_{z}\left(x_{0}, y_{0}\right) d x_{0} d y_{0}
$$

where $(x, y)$ and $\left(x_{0}, y_{0}\right)$ are arbitrary points inside the periphery of the circuit boundary, which is the area designated by $D$ in Fig. 2.1. $\mathcal{G}\left(x, y \mid x_{0}, y_{0}\right)$ is a solution of $2 \mathrm{D}$ homogeneous Helmholtz equation

$$
\left(\nabla_{t}^{2}+k^{2}\right) \mathcal{G}\left(x, y \mid x_{0}, y_{0}\right)=0
$$

subject to the open-circuit boundary condition $\partial \mathcal{G} / \partial n=0$ along the contour $C$. These Green's functions are known for several regular shapes in the form of infinite series, such as rectangles [36], circles [36], several types of triangular segments [39], and circular and annular sectors and rings [37], [40]. A rather exhaustive list of these Green's function is given in [40] and can be consulted for further information. For irregular shapes and many other regular shapes, however, Green's functions are 

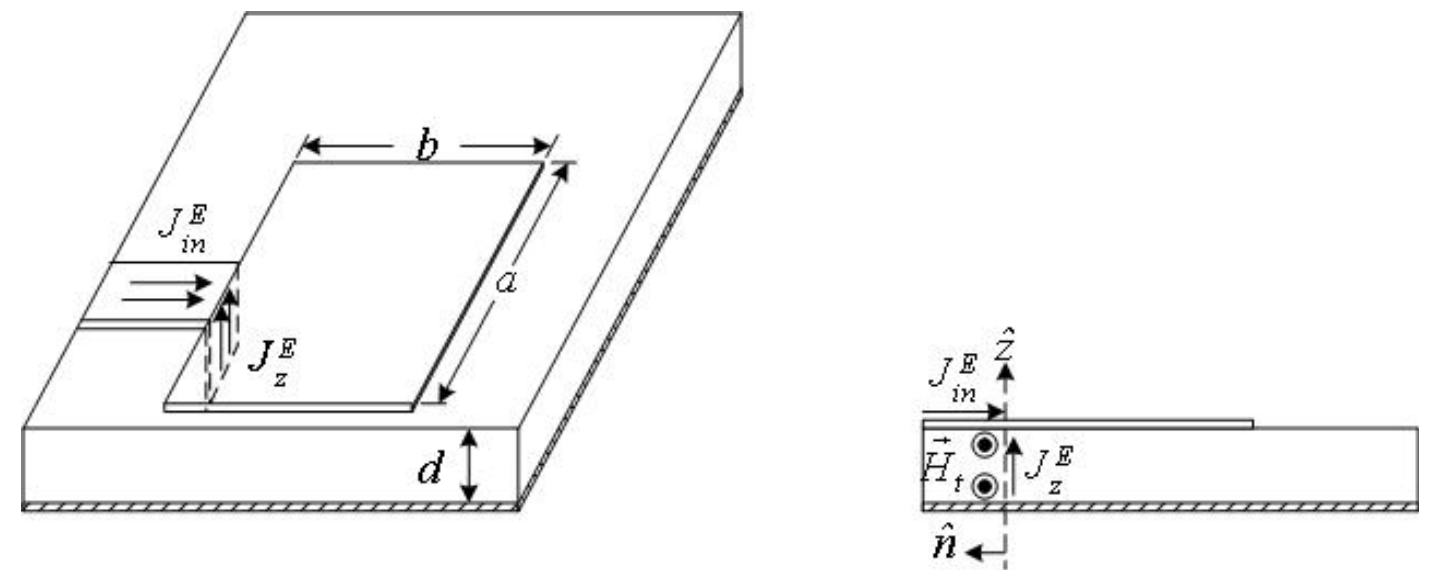

Figure 2.2: Equivalence between coplanar feedline and vertical probe currents [37].

not available analytically and numerical methods such as Contour Integral Method are used instead [36]. Since Contour Integral Method is one of the main focuses of this thesis, it will be addressed in detail in an upcoming section.

Microstrip line feeds for planar circuits can be approximated by a $z$-directed electric current sheet on the boundary of the circuit as illustrated in Fig. 2.2 [37]. The equivalency between the two can be established by noticing that:

$$
J_{z}^{E}=\hat{n} \times \vec{H}_{t} \quad, \quad J_{i n}^{E}=\hat{z} \times \vec{H}_{t}
$$

where $J_{z}^{E}$ and $J_{i n}^{E}$ are defined in Fig. 2.2. From 2.13 it can be seen that $\left|J_{z}^{E}\right|=\left|J_{i n}^{E}\right|$. If $w_{j}$ is the effective width of the microstrip line feeding the $j^{\text {th }}$ edge port, the input current at that port can be defined as $\bar{I}_{j}^{E}=w_{j} J_{z}^{E}$ [37], [38]. Once the equivalence between $J_{z}^{E}$ and $J_{i n}^{E}$ has been established, equation 2.11 can be adapted for the voltage along the periphery of the circuit when the input current is injected into the circuit via coplanar microstrip lines connected to the edge ports as follows:

$$
V^{E}(s)=\oint_{C} \mathcal{G}\left(s \mid s_{0}\right) J^{E}\left(s_{0}\right) d s_{0}
$$

where $s$ and $s_{0}$ denote distances along the contour $C$ in counterclockwise direction. $J^{E}\left(s_{0}\right)$ is the input current flowing into the circuit from an external port at $s_{0}$ and $V^{E}(s)$ is the voltage induced at the point $s$. If the periphery of the circuit is divided into $N^{E}$ smaller segments, where each segment is approximated by a straight line segment, the closed contour integral can be broken down to sum of 


\section{CHAPTER 2. MULTIPORT NETWORK MODEL}

$N^{E}$ line integrals as:

$$
V^{E}(s)=\sum_{j=1}^{N^{E}} \int_{w_{j}} \mathcal{G}\left(s \mid s_{0}\right) J^{E}\left(s_{0}\right) d s_{0}
$$

Terminal voltage for the $i^{\text {th }}$ edge port defined in 2.10, can be written as:

$$
\bar{V}_{i}^{E}=\int_{w_{i}} V^{E}(s) d s / w_{i}
$$

from which we will have:

$$
\begin{aligned}
\bar{V}_{i}^{E} & =\frac{1}{w_{i}} \sum_{j=1}^{N^{E}} \iint_{w_{i}} \int_{w_{j}} \mathcal{G}\left(s \mid s_{0}\right) J^{E}\left(s_{0}\right) d s_{0} d s \\
& \cong \sum_{j=1}^{N^{E}} \frac{\bar{I}_{j}^{E}}{w_{i} w_{j}} \iint_{w_{i}} \mathcal{G}\left(s \mid s_{0}\right) d s_{0} d s
\end{aligned}
$$

where it has been assumed that $J^{E}\left(s_{0}\right)$ does not change appreciably over the width of the port and hence one can write:

$$
\bar{I}_{j}^{E}=\int_{w_{j}} J^{E}\left(s_{0}\right) d s_{0} \cong w_{j} \bar{J}_{j}^{E}
$$

where $\bar{J}_{j}^{E}$ is the value of $J^{E}\left(s_{0}\right)$ at the midpoint of the $j^{\text {th }}$ segment. Impedance matrix elements, i.e. $\mathbf{Z}_{i j}^{E}$ can be readily evaluated from 2.17 as:

$$
\mathbf{Z}_{i j}^{E}=\left.\frac{\bar{V}_{i}^{E}}{\bar{I}_{j}^{E}}\right|_{\bar{I}_{i}^{E}=0, i \neq j} \equiv \frac{1}{w_{i} w_{j}} \iint_{w_{i}} \mathcal{G}\left(s \mid s_{j}\right) d s_{0} d s
$$

In $2.19, d s_{0}$ and $d s$ correspond to integrations over the width of the $j^{\text {th }}$ and $i^{\text {th }}$ segments, respectively. $\bar{I}_{i}^{E}=0, i \neq j$ also indicates that except for the $j^{\text {th }}$ port, all other ports are open-circuited. 


\subsection{Derivation of Green's Function in terms of Eigenfunctions}

Equations 2.2 and 2.7 fully characterize the planar structure in the absence of any external connection. Therefore, the eigenfunctions of the planar structure are nontrivial solutions of the following homogeneous wave equation subject to open-circuit boundary condition:

$$
\begin{aligned}
& \left(\nabla_{t}^{2}+k^{2}\right) V=0 \quad(\text { in } D) \\
& \frac{\partial V}{\partial n}=0 \quad(\text { on } C)
\end{aligned}
$$

It can be shown that in order for a nonzero solution to exist, the parameter $k$ has to take on one of an infinite number of discrete values, known as eigenvalues. The infinity and discreteness of this set stems from the fact that the dielectric filling is homogeneous and the structure is spatially confined [41]. These eigenvalues correspond to discrete resonance frequencies of the structure and their corresponding field solutions are called eigenfunctions. If $k_{n}$ and $\phi_{n}$ denote these eigenvalues and eigenfunctions, respectively, it can be readily shown that $k_{n}$ 's are the stationary values of the following variational expression [36]:

$$
k_{n}^{2}=\frac{\iint_{D}\left|\nabla \phi_{n}\right|^{2} d S}{\iint_{D}\left|\phi_{n}\right|^{2} d S}
$$

where $\phi_{n}$ is the solution (eigenfunction) corresponding to $k_{n}$. Note that $d S$ denotes a surface integration and should not be confused with $d s$ used to denote line integration. It can also be shown that these eigenfunctions form an orthogonal set [40], meaning that:

$$
\iint_{D} \phi_{m} \phi_{n}^{*} d S=\delta_{m n}
$$

provided that they are normalized. $\delta_{m n}$ is the Kronecker's delta function defined as:

$$
\delta_{m n}= \begin{cases}1 & m=n \\ 0 & m \neq n\end{cases}
$$




\section{CHAPTER 2. MULTIPORT NETWORK MODEL}

Any inhomogeneous solution to 2.20, including the Green's function, can be expanded in terms of $\phi_{n}$ 's as follows [41]:

$$
\mathcal{G}\left(x, y \mid x_{0}, y_{0}\right)=\sum_{n=1}^{\infty} A_{n} \phi_{n}
$$

where $A_{n}$ 's are constant coefficients given as [40]:

$$
A_{n}=j \omega \mu d \frac{\phi_{n}^{*}\left(x_{0}, y_{0}\right)}{k_{n}^{2}-k^{2}}
$$

Hence, the Green's function in terms of the eigenfunctions can be written as:

$$
\mathcal{G}\left(x, y \mid x_{0}, y_{0}\right)=j \omega \mu d \sum_{n=1}^{\infty} \frac{\phi_{n}^{*}\left(x_{0}, y_{0}\right) \phi_{n}(x, y)}{k_{n}^{2}-k^{2}}
$$

From which the elements of edge impedance matrix can be found in terms of eigenvalues and eigenfunctions as follows [36]:

$$
\mathbf{Z}_{i j}^{E}=\frac{1}{w_{i} w_{j}} \int_{w_{i}} \int_{w_{j}} j \omega \mu d \sum_{n=1}^{\infty} \frac{\phi_{n}^{*}\left(s_{0}\right) \phi_{n}(s)}{k_{n}^{2}-k^{2}} d s_{0} d s
$$

where $d s_{0}$ and $d s$ correspond to integrations over the width of the $j^{\text {th }}$ and $i^{\text {th }}$ segments, respectively. The eigenfunctions can be found analytically only if the internal angle at each vertex is a submultiple of $\pi$ [40], such as rectangles and some triangles. They can also be computed analytically for circular and annular sectors and rings [40]. For some irregular shapes, segmentation and desegmentation methods of analysis can be used to compute their impedance matrices from the impedance matrices of regular shapes.

Segmentation was originally proposed by Okoshi [36], [42], [43] for irregularly shaped planar circuits which could be divided into smaller segments having regular shapes. An example is shown in Fig. 2.3, where a ring-shaped geometry is broken down into four rectangular segments. The smaller segments can be easily characterized by their impedance matrices, which in turn are connected using network modeling techniques to give the overall impedance matrix of the composite structure. At first, segmentation method was developed using S-parameters for characterizing individual segments [43], but later it was found that Z-matrix formulation was more efficient [44].

Desegmentation can be roughly considered complementary to the segmentation method. Though some planar circuits cannot be divided into smaller segments 
CHAPTER 2. MULTIPORT NETWORK MODEL

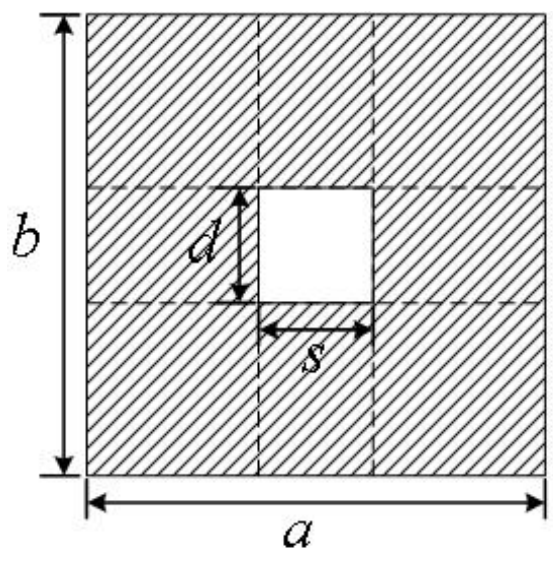

(a)

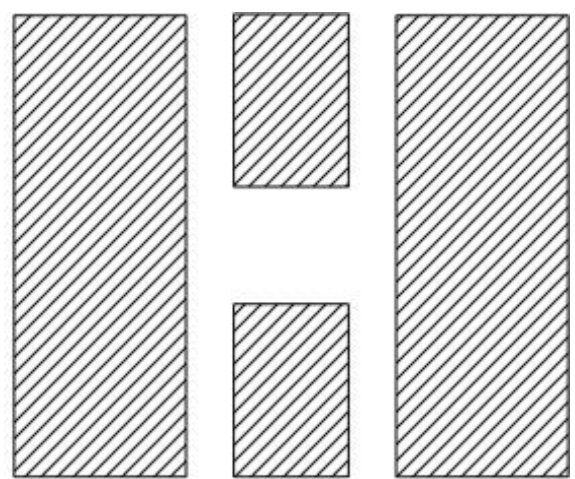

(b)

Figure 2.3: Concept of segmentation [37].

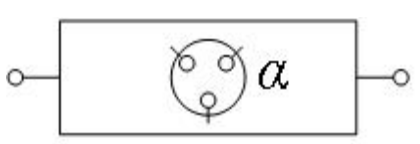

(a)

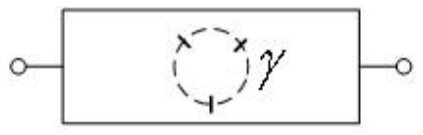

(b)

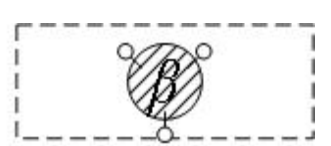

(c)

Figure 2.4: Concept of desegmentation [37]

having regular shapes, they can be converted into a regular shape by adding a smaller regularly-shaped segment. An example is the rectangular patch with a circular hole, illustrated in Fig. 2.4 [37]. The planar circuit in Fig. 2.4a can be obtained by desegmenting the circular patch in Fig. 2.4c from the rectangular patch in Fig. 2.4b. It is important to notice that some structures can be analyzed by both methods. For example, if a rectangular segment is added to the structure in Fig. 2.3, the resulting structure is also rectangular, hence this planar circuit can be analyzed by desegmentation as well as segmentation.

Another approach to analysis and design of irregularly shaped planar circuits is based on numerical computation of eigenfunctions and eigenvalues, from which the Green's function and hence equivalent multiport network model can be obtained. Numerical computation of eigenfunctions and eigenvalues involves solving two-dimensional Helmholtz equation subject to open-circuit boundary condition or equivalently TE-modes in a hollow metallic waveguide with the same cross sec- 
tion [36]. This can be done by variety of numerical techniques for electromagnetic structures, among which the most popular are:

- Finite-Difference Method [45]

- Finite-Element Method [46]

- Point-Matching Method [47]

- Rayleigh-Ritz Method [48], [49]

Numerical solution of Helmholtz equation requires field solution over the entire area of the planar circuit and hence can be quite time-consuming. An alternative approach is to convert the wave equation into an integral equation along the circuit periphery [36], from which equivalent circuit parameters can be derived. This method was originally developed by Okoshi for planar circuits [36] and is called the "Contour-Integral Method" or "Boundary-Integral Method". One of the contributions of this thesis is derivation of a novel contour-integral equation, which along with the traditional contour-integral equation developed by Okoshi, will be derived from vector Green's theorem in the next section.

\subsection{Contour-Integral Equation}

Contour-integral equation for MNM of irregular planar circuits was originally derived using Weber's solution with cylindrical waves [36], [42]. The derivation based on vector Green's theorem [41], [50], is more general and will be presented here. In addition, the new contour integral equation that will be presented in this section cannot be derived by Weber's solution.

Green's theorems are symmetrical mathematical relationships between two functions [41]. Reciprocity theorem is a special case where the two functions signify fields associated with two different sources and hence have physical meanings, whereas no physical interpretation is necessarily attached to the functions in Green's theorems. We start with the scalar Green's theorem based on the following identity for any two well-behaved scalar functions $\psi$ and $\phi$ :

$$
\nabla \cdot(\psi \nabla \phi)=\psi \nabla^{2} \phi+\nabla \psi \cdot \nabla \phi
$$




\section{CHAPTER 2. MULTIPORT NETWORK MODEL}

By integrating both sides of 2.28 throughout a region and applying the divergence theorem to the Left-Hand Side (LHS), Green's first identity can be obtained as [41]:

$$
\oint_{S} \psi \frac{\partial \phi}{\partial n} d S=\iint_{D} \int\left(\psi \nabla^{2} \phi+\nabla \psi \cdot \nabla \phi\right) d v
$$

where as shown in Fig. 2.5, $S$ is a closed surface and $D$ is the region inside $S$. Interchanging $\psi$ and $\phi$ in 2.29 and subtracting the resulting equation from 2.29, provides us with Green's second identity or Green's Theorem [41]:

$$
\oint_{S}\left(\psi \frac{\partial \phi}{\partial n}-\phi \frac{\partial \psi}{\partial n}\right) d S=\iiint_{D}\left(\psi \nabla^{2} \phi-\phi \nabla^{2} \psi\right) d v
$$

which can be interpreted as the reciprocity between the scalar fields $\psi$ and $\phi . \hat{n}$ and $\partial / \partial n$ denote the outward normal to the surface enclosing the region of the problem $S$ and the derivative in that direction. By definition we have $\partial \phi / \partial n=\nabla \phi \cdot \hat{n}$. Weber's solution in [36] has been derived from Green's second identity by solving the reciprocal problem. This is the approach we will also follow here, but instead we will use vector Green's theorem. The vector analogue of 2.28 can be written as [41]:

$$
\nabla \cdot(\vec{A} \times \nabla \times \vec{B})=\nabla \times \vec{A} \cdot \nabla \times \vec{B}-\vec{A} \cdot \nabla \times \nabla \times \vec{B}
$$

where $\vec{A}$ and $\vec{B}$ are two well-bahaving but otherwise arbitrary vector functions. In a like manner to the scalar case, by integrating both sides of 2.31 and applying the divergence theorem to the LHS, we obtain vector analogue of Green's first identity [41]:

$$
\oint_{S}(\vec{A} \times \nabla \times \vec{B}) \cdot \hat{n} d S=\iint_{D} \int(\nabla \times \vec{A} \cdot \nabla \times \vec{B}-\vec{A} \cdot \nabla \times \nabla \times \vec{B}) d v
$$

Again, interchanging $\vec{A}$ and $\vec{B}$ and subtracting the resulting equation from 2.32, yields vector analogue of Green's second identity or vector Green's theorem [41]:

$$
\begin{aligned}
& \oint_{S}(\vec{A} \times \nabla \times \vec{B}-\vec{B} \times \nabla \times \vec{A}) \cdot \hat{n} d S= \\
& \iint_{D} \int(\vec{B} \cdot \nabla \times \nabla \times \vec{A}-\vec{A} \cdot \nabla \times \nabla \times \vec{B}) d v
\end{aligned}
$$

It can be seen that with $\vec{A}=\vec{E}^{a}$ and $\vec{B}=\vec{E}^{b}$, where $\vec{E}^{a}$ and $\vec{E}^{b}$ are the electric fields due to two arbitrary sources $a$ and $b$, equation 2.33 is in fact the statement of reciprocity between those two sources in a homogeneous medium. 


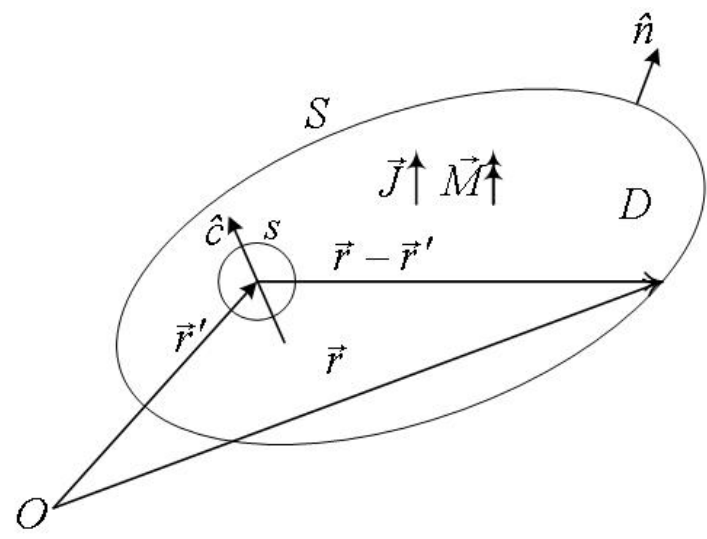

(a)

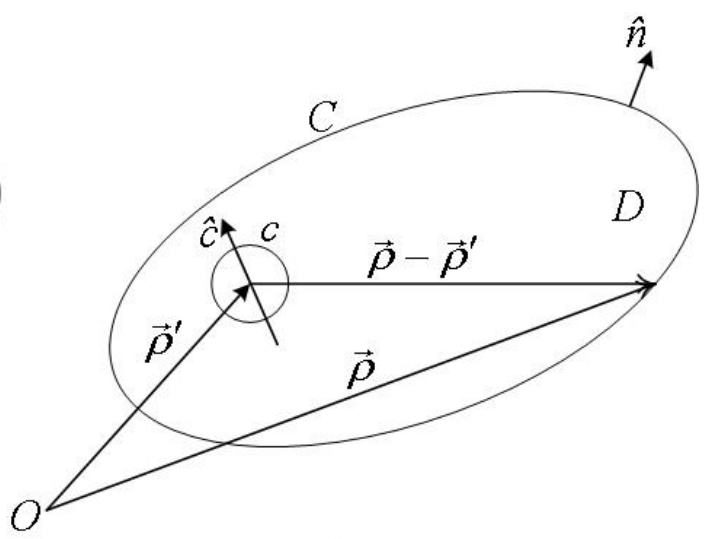

(b)

Figure 2.5: Region to which Green's theorem is applied: a) Three-dimensional region [41]. b) Two dimensional region for contour-integral equations.

Suppose that we are interested in finding the electric field $\vec{E}$ at an arbitrary point $r^{\prime}$ inside the region denoted by $D$, enclosed by the surface $S$ and containing impressed electric and magnetic sources $\vec{J}$ and $\vec{M}$, respectively, as shown in Fig. 2.5a. According to the Uniqueness theorem [41], the field cannot be specified uniquely unless the boundary conditions are known. These boundary conditions could be either $\hat{n} \times \vec{E}$ or $\hat{n} \times \vec{H}$ or a combination of the two on $S$. Instead of solving this problem directly, the reciprocal problem, i.e. finding the field on the boundary due to a source at $r^{\prime}$, will be solved. The field due to the point source of unit strength at $r^{\prime}$ is called the Green's function and will be denoted by $\vec{G}$. As will be seen shortly, different choices for $\vec{G}$ will result in different contour-integral equations. $\vec{G}_{1}$ and $\vec{G}_{2}$ will be used to denote the two choices.

First we choose $\vec{G}_{1}=\hat{c} \phi$, where $\hat{c}$ is an arbitrary constant unit vector and:

$$
\phi=\frac{e^{-j k\left|\vec{r}-\vec{r}^{\prime}\right|}}{\left|\vec{r}-\vec{r}^{\prime}\right|}
$$

is the Green's function for the vector potential of an electric dipole $I \vec{\ell}=4 \pi \hat{c}[41]$. It can be easily shown that anywhere but at $\vec{r}=\vec{r}^{\prime}$ :

$$
\left\{\begin{array}{l}
\nabla \times \vec{G}_{1}=\nabla \phi \times \hat{c} \\
\nabla \times \nabla \times \vec{G}_{1}=\hat{c} k^{2} \phi+\nabla(\hat{c} \cdot \nabla \phi)
\end{array}\right.
$$




\section{CHAPTER 2. MULTIPORT NETWORK MODEL}

By substituting $\vec{A}=\vec{E}$ and $\vec{B}=\vec{G}_{1}$ in 2.33 and making use of 2.35, we have:

$$
\begin{aligned}
& \iint_{D} \int\left[\hat{c} \phi \cdot \nabla \times \nabla \times \vec{E}-k^{2} \phi \hat{c} \cdot \vec{E}+\vec{E} \cdot \nabla(\hat{c} \cdot \nabla \phi)\right] d v= \\
& \oint \oint_{S}[\vec{E} \times(\nabla \phi \times \hat{c})-\hat{c} \phi \times \nabla \times \vec{E}] \cdot \hat{n} d S
\end{aligned}
$$

Both sides of 2.36 can be simplified by noticing that $\vec{E}$ satisfies Maxwell's equation as well as Helmholtz equation. For the Right-Hand Side (LHS) we have:

$$
\text { LHS of } 2.36=\hat{c} \cdot \iint_{D} \int\left(\nabla \phi \frac{\rho_{v}}{\varepsilon}-j \omega \mu \phi \vec{J}-\phi \nabla \times \vec{M}\right) d v-\hat{c} \cdot \oint_{S} \oint_{S} \nabla \phi \hat{n} \cdot \vec{E} d S
$$

where $\nabla \cdot \vec{E}=\rho_{v} / \varepsilon$. $\hat{c}$ can also be factored out of the Right-Hand Side (RHS) of 2.36 by making use of the following two identities:

$$
\left\{\begin{array}{l}
{[\vec{E} \times(\nabla \phi \times \hat{c})] \cdot \hat{n}=\hat{c} \cdot[\nabla \phi \times(\vec{E} \times \hat{n})]} \\
(\hat{c} \phi \times \nabla \times \vec{E}) \cdot \hat{n}=\hat{c} \cdot(j \omega \mu \phi \hat{n} \times \vec{H}+\phi \hat{n} \times \vec{M})
\end{array}\right.
$$

From which and 2.36 we have:

$$
\text { RHS of } 2.36=\hat{c} \cdot \oint_{S}[(\hat{n} \times \vec{E}) \times \nabla \phi-j \omega \mu \phi \vec{n} \times \vec{H}-\phi \hat{n} \times \vec{M}] d S
$$

Combining 2.39 and 2.37, by noticing that $\hat{c}$ is an arbitrary vector, and by using the following identity:

$$
\iint_{D} \int \nabla \times \vec{M} \phi d v=\oint_{S} \hat{n} \times \vec{M} \phi d S+\iiint_{D} \vec{M} \times \nabla \phi d v
$$

we will have:

$$
\begin{aligned}
& \oint_{S}[(\hat{n} \times \vec{E}) \times \nabla \phi+\nabla \phi \hat{n} \cdot \vec{E}-j \omega \mu \phi \vec{n} \times \vec{H}] d S= \\
& \iint_{D} \int\left(\nabla \phi \frac{\rho_{v}}{\varepsilon}-j \omega \mu \phi \vec{J}-\vec{M} \times \nabla \phi\right) d v
\end{aligned}
$$

It is important to recognize that 2.41 is not valid at $\vec{r}=\vec{r}^{\prime}$, at which we desire $\vec{E}$. In order to extract this singularity, a sphere of radius $r_{0}$ is circumscribed about 


\section{CHAPTER 2. MULTIPORT NETWORK MODEL}

the point $\vec{r}=\vec{r}^{\prime}$ (sphere $s$ in Fig. 2.5a). Equation 2.41 is valid at all points inside the region enclosed between the spheres $S$ and $s$, except at the sources $\vec{J}$ and $\vec{M}$. In the limit when $r_{0}$ shrinks to zero, the contribution of the sphere $s$ to the RHS of 2.41 can be computed analytically by substituting for $\phi$ and $\nabla \phi$ from 2.34 [50]. Consequently we will have [50]:

$$
\begin{aligned}
\vec{E}\left(\vec{r}^{\prime}\right)= & -\frac{1}{4 \pi} \oint_{\oint_{S}}[(\hat{n} \times \vec{E}) \times \nabla \phi+\nabla \phi \hat{n} \cdot \vec{E}-j \omega \mu \phi \vec{n} \times \vec{H}] d S+ \\
& \frac{1}{4 \pi} \iint_{D} \int\left(\nabla \phi \frac{\rho_{v}}{\varepsilon}-j \omega \mu \phi \vec{J}-\vec{M} \times \nabla \phi\right) d v
\end{aligned}
$$

This is a formula for calculating $\vec{E}$ at $\vec{r}^{\prime}$ in terms of normal component of $\vec{E}$ and tangential components of both $\vec{E}$ and $\vec{H}$ on $S$ and the sources inside the region $D$. Since both tangential and normal components of $\vec{E}$ on $S$ are required, $\vec{E}$ has to be continuous on $S$. Furthermore, since $\vec{H}$ is the first derivative of $\vec{E}, \vec{E}$ is required to have continuous first derivatives on $S$ as well [41]. This is a sever restriction on the usefulness of 2.42. As it will be shown shortly, nonetheless, this equation is the basis for the traditional contour-integral equation.

Now let

$$
\vec{B}=\vec{G}_{2}=\nabla \times \vec{G}_{1}
$$

which is the magnetic field of an electric dipole $I \vec{\ell}=4 \pi \hat{c}$ [41]. It can be easily shown that anywhere but at $\vec{r}=\vec{r}^{\prime}$ :

$$
\left\{\begin{array}{l}
\nabla \times \vec{G}_{2}=\hat{c} k^{2} \phi+\nabla(\hat{c} \cdot \nabla \phi) \\
\nabla \times \nabla \times \vec{G}_{2}=k^{2} \vec{G}_{2}
\end{array}\right.
$$

Substituting $\vec{A}=\vec{E}$ and $\vec{B}=\vec{G}_{2}$ in 2.33 yields:

$$
\begin{aligned}
& \oint_{S}\left(\vec{E} \times \nabla \times \vec{G}_{2}-\vec{G}_{2} \times \nabla \times \vec{E}\right) \cdot \hat{n} d S= \\
& \iint_{D} \int\left(\vec{G}_{2} \cdot \nabla \times \nabla \times \vec{E}-\vec{E} \cdot \nabla \times \nabla \times \vec{G}_{2}\right) d v
\end{aligned}
$$

By following a similar procedure and making use of 2.44, it can be shown that:

$$
\text { LHS of } 2.45=\hat{c} \cdot \oint_{S}\left[k^{2} \phi \hat{n} \times \vec{E}+(\nabla \times \vec{E} \cdot \hat{n}) \nabla \phi-\nabla \phi \times(\hat{n} \times \nabla \times \vec{E})\right] d S
$$




$$
\text { RHS of } 2.45=-\hat{c} \cdot \iint_{D} \int[j \omega \mu(\vec{J} \times \nabla \phi)-(\nabla \times \vec{M}) \times \nabla \phi] d v
$$

The singularity can be extracted by applying the same method. Enclosing the point $\vec{r}=\vec{r}^{\prime}$ by a sphere of radius $r_{0}$, and comparing $2.45,2.46$, and 2.47 in the absence of any sources, i.e. $\vec{J}=\vec{M}=0$, it can be seen that:

$$
\begin{aligned}
& \oint_{S} \oint_{S}\left(\vec{E} \times \nabla \times \vec{G}_{2}-\vec{G}_{2} \times \nabla \times \vec{E}\right) \cdot \hat{n} d S= \\
& \hat{c} \cdot \oint_{\oint_{S}}\left[k^{2} \phi \hat{n} \times \vec{E}+(\nabla \times E \cdot \hat{n}) \nabla \phi-\nabla \phi \times(\hat{n} \times \nabla \times \vec{E})\right] d S
\end{aligned}
$$

which upon extraction of the singularity becomes:

$$
-4 \pi \hat{c} \cdot\left[\nabla^{\prime} \times \vec{E}\left(\vec{r}^{\prime}\right)\right]=\oint_{S}\left(\vec{E} \times \nabla \times \vec{G}_{2}-\vec{G}_{2} \times \nabla \times \vec{E}\right) \cdot \hat{n} d S
$$

Equation 2.49 can be cast into the following more insightful form by simple algebraic manipulations:

$$
\hat{c} \cdot \vec{H}\left(\vec{r}^{\prime}\right)=\frac{1}{j \omega \mu} \frac{1}{4 \pi} \oint_{S} \nabla \times \vec{G}_{2} \cdot(\hat{n} \times \vec{E}) d S-\frac{1}{4 \pi} \oint_{S} \oint_{S} \vec{G}_{2} \cdot(\hat{n} \times \vec{H}) d S
$$

which is an expression for $\vec{H}\left(\vec{r}^{\prime}\right)$ in terms of tangential components of both $\vec{E}$ and $\vec{H}$. In this case $\vec{E}$ does not have to be continuous on $S$. Note that the RHS of 2.50 is a simple rearrangement of the LHS of 2.48 .

Similar steps can be followed for two-dimensional planar circuits. The only difference is that instead of spherical wave function, cylindrical wave function must be used. In other words one has to choose $\phi=H_{0}^{(2)}\left(k\left|\vec{\rho}-\vec{\rho}^{\prime}\right|\right)$, where $H_{n}^{(2)}(\cdot)$ is the $n^{\text {th }}$ order Hankel function of the second kind. In order to extract the singularity, a circle of radius $\rho_{0}$ is circumscribed about the point $\vec{\rho}=\vec{\rho}^{\prime}$ (Fig. 2.5b) and then the contribution of this circle to the surface integral is calculated analytically by approximating $\phi$ and $\nabla \phi$ by the small argument expressions for $H_{0}^{(2)}\left(k\left|\vec{\rho}-\vec{\rho}^{\prime}\right|\right)$ when $\rho_{0}$ tends to zero. In the absence of any sources we will have the following contour-integral equations:

$$
\vec{E}\left(\vec{\rho}^{\prime}\right)=-\frac{1}{4 j} \oint_{C}[(\hat{n} \times \vec{E}) \times \nabla \phi+\nabla \phi \hat{n} \cdot \vec{E}-j \omega \mu \phi \vec{n} \times \vec{H}] d s
$$




\section{CHAPTER 2. MULTIPORT NETWORK MODEL}

$$
\hat{c} \cdot \vec{H}\left(\vec{\rho}^{\prime}\right)=-\frac{1}{4 \omega \mu} \oint_{C} \nabla \times \vec{G}_{2} \cdot(\hat{n} \times \vec{E}) d s-\frac{1}{4 j} \oint_{C} \vec{G}_{2} \cdot(\hat{n} \times \vec{H}) d s
$$

One of the contributions of this thesis is 2.52 , which is a new form of contour-integral equation.

To show that 2.51 is in fact the traditional contour-integral equation, without loss of generality, we assume that the origin of the coordinates in Fig. 2.5b coincides with the observation point, in other words $\vec{\rho}^{\prime}=0$. We notice that thin planar structures support only $T M^{z}$ modes with $\vec{E}=\hat{z} E_{z}$. From 2.4 and 2.6 it can also be seen that:

$$
\frac{\partial E_{z}}{\partial n}=\hat{n} \cdot \nabla E_{z}=-j \omega \mu\left(\hat{n} \cdot \vec{J}_{s u r f}\right)=j \omega \mu \hat{z} \cdot \hat{n} \times \vec{H}
$$

where $\hat{n}$ is the outward normal to the boundary, as shown in Fig. 2.1. In this case 2.51 reduces to:

$$
\begin{aligned}
E_{z}\left(\vec{\rho}^{\prime}\right) & =-\frac{1}{4 j} \hat{z} \cdot \oint_{C}\left[(\hat{n} \times \hat{z}) E_{z} \times \nabla \phi+\nabla \phi \hat{n} \cdot \hat{z} E_{z}-j \omega \mu \phi \vec{n} \times \vec{H}\right] d s \\
& =-\frac{1}{4 j} \oint_{C}\left(\frac{\partial H_{0}^{(2)}(k \rho)}{\partial n} E_{z}-H_{0}^{(2)}(k \rho) \frac{\partial E_{z}}{\partial n}\right) d s
\end{aligned}
$$

where use has been made of the fact that

$$
\hat{z} \cdot(\hat{n} \times \hat{z}) \times \nabla \phi=\hat{n} \cdot \nabla \phi=\frac{\partial \phi}{\partial n}
$$

If one substitutes $V=d E_{z}$ in 2.54, the result will be equation (A3.8) in [36]. Equation 2.54 gives potential at any point inside the contour $C$ in terms of the potential and its normal derivative on the boundary. It can be shown that the potential at any point on the boundary can also be written in terms of the potential and its normal derivative on the boundary as follows (Appendix B):

$$
V(s)=-\frac{1}{2 j} \oint_{C}\left(\frac{\partial H_{0}^{(2)}(k \rho)}{\partial n} V-H_{0}^{(2)}(k \rho) \frac{\partial V}{\partial n}\right) d s_{0}
$$

Combining 2.56 with 2.9, we will have [36]:

$$
V(s)=\frac{1}{2 j} \oint_{C}\left(k \cos \theta H_{1}^{(2)}(k \rho) V\left(s_{0}\right)+H_{0}^{(2)}(k \rho) \frac{j \omega \mu}{d} J^{E}\left(s_{0}\right)\right) d s_{0}
$$



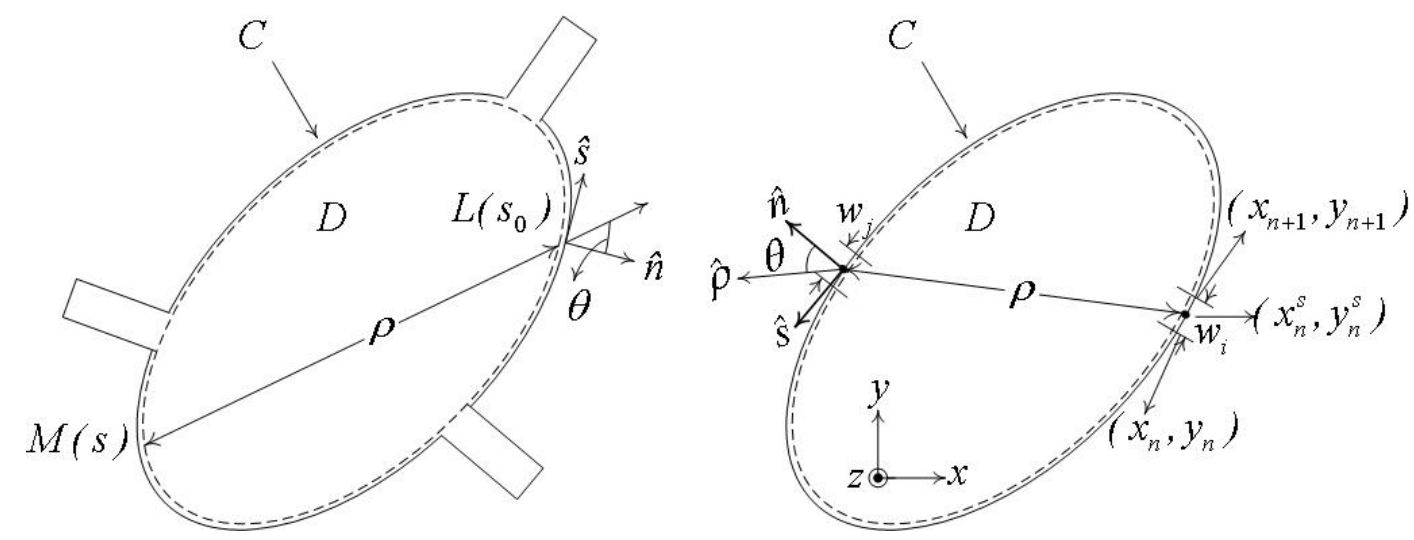

Figure 2.6: Symbols used in a) contour-integral equation, b) numerical analysis [36].

where

$$
\frac{\partial H_{0}^{(2)}(k \rho)}{\partial n}=\hat{n} \cdot \nabla H_{0}^{(2)}(k \rho)=\hat{n} \cdot \hat{\rho} \frac{\partial H_{0}^{(2)}(k \rho)}{\partial \rho}
$$

and $\hat{n} \cdot \hat{\rho}=\cos \theta . \theta$ is defined in Fig. 2.6a as the angle between the line joining points $M(s)$ and $L\left(s_{0}\right)$ and the normal to $C$ at $L\left(s_{0}\right) . J^{E}\left(s_{0}\right)$ is the input current flowing into the circuit at $L\left(s_{0}\right)$.

\subsubsection{Derivation of a Novel Contour-Integral Equation}

Equation 2.52 gives the magnetic field at any point inside the contour $C$ in terms of the tangential components of electric and magnetic fields on $C$. Similar to 2.51, equation 2.52 can also be modified for the case when the observation point is on the boundary. The procedure, however, is not as straightforward as the previous case. As a matter of fact, the procedure outlined in Appendix B cannot be directly applied to 2.52 . We start from 2.52 by noticing that for thin planar structures $\vec{E}=\hat{z} E_{z}$ and $H_{z}=0$. If we define

$$
M^{\text {wall }} \equiv(\vec{E} \times \hat{n}) \cdot \hat{s}
$$

we will have:

$$
\hat{c} \cdot \vec{H}\left(\vec{\rho}^{\prime}\right)=\frac{1}{4 \omega \mu} \oint_{C}\left(\nabla \times \vec{G}_{2} \cdot \hat{s}\right) M^{\text {wall }} d s-\frac{1}{4 j} \oint_{C}\left(\vec{G}_{2} \cdot \hat{z}\right)(\hat{s} \cdot \vec{H}) d s
$$




\section{CHAPTER 2. MULTIPORT NETWORK MODEL}

Without loss of generality it will be assumed that the origin of the coordinates in Fig. $2.5 \mathrm{~b}$ coincides with the observation point, in other words $\vec{\rho}^{\prime}=0$. Hence by remembering that $\vec{G}_{2}=\nabla \times \hat{c} \phi$, where $\phi=H_{0}^{(2)}(k \rho)$, one can write:

$$
\begin{gathered}
\vec{G}_{2} \cdot \hat{z}=\nabla \times \hat{c} \phi \cdot \hat{z}=\hat{z} \cdot(\nabla \phi \times \hat{c})=(\hat{c} \cdot \hat{\varphi}) \frac{\partial \phi}{\partial \rho} \\
\nabla \times \vec{G}_{2} \cdot \hat{s}=\nabla \times \nabla \times \hat{c} \phi \cdot \hat{s}=(\hat{c} \cdot \hat{s}) k^{2} \phi+\frac{\partial}{\partial s}\left(\hat{c} \cdot \hat{\rho} \frac{\partial \phi}{\partial \rho}\right)
\end{gathered}
$$

where $\hat{\varphi}=\hat{z} \times \hat{\rho}$. Hence:

$$
\begin{aligned}
\hat{c} \cdot \vec{H}\left(\vec{\rho}^{\prime}\right)= & \frac{k^{2}}{4 \omega \mu} \oint_{C}(\hat{c} \cdot \hat{s}) \phi M^{\text {wall }} d s+\frac{1}{4 \omega \mu} \oint_{C} \frac{\partial}{\partial s}\left(\hat{c} \cdot \hat{\rho} \frac{\partial \phi}{\partial \rho}\right) M^{\text {wall }} d s+ \\
& -\frac{1}{4 j} \oint_{C}(\hat{c} \cdot \hat{\varphi}) \frac{\partial \phi}{\partial \rho}(\hat{s} \cdot \vec{H}) d s
\end{aligned}
$$

Since the integration is performed on a closed contour, the second integral in 2.63 can be written as:

$$
\begin{aligned}
\oint_{C} \frac{\partial}{\partial s}\left(\hat{c} \cdot \hat{\rho} \frac{\partial \phi}{\partial \rho}\right) M^{\text {wall }} d s & =(\hat{c} \cdot \hat{\rho}) \frac{\partial \phi}{\partial \rho} M^{\text {wall }}-\oint_{C}(\hat{c} \cdot \hat{\rho}) \frac{\partial \phi}{\partial \rho} \frac{\partial M^{\text {wall }}}{\partial s} d s \\
& =-\oint_{C}(\hat{c} \cdot \hat{\rho}) \frac{\partial \phi}{\partial \rho} \frac{\partial M^{\text {wall }}}{\partial s} d s
\end{aligned}
$$

Substituting 2.64 back into 2.63 yields:

$$
\begin{aligned}
\hat{c} \cdot \vec{H}\left(\vec{r}^{\prime}\right)= & \frac{k^{2}}{4 \omega \mu} \oint_{C}(\hat{c} \cdot \hat{s}) \phi M^{\text {wall }} d s-\frac{1}{4 \omega \mu} \oint_{C}(\hat{c} \cdot \hat{\rho}) \frac{\partial \phi}{\partial \rho} \frac{\partial M^{\text {wall }}}{\partial s} d s+ \\
& -\frac{1}{4 j} \oint_{C}(\hat{c} \cdot \hat{\varphi}) \frac{\partial \phi}{\partial \rho}(\hat{s} \cdot \vec{H}) d s
\end{aligned}
$$

Now the procedure outlined in Appendix B can be applied to 2.65. Since only the tangential component of magnetic field on the boundary will be needed, we will assume $\hat{c}=\hat{s}_{i}$, where $\hat{s}_{i}$ is the tangent to the boundary at the point $P^{\prime}$ in Fig. B.1. By substituting $\phi=H_{0}^{(2)}(k \rho)$, breaking down the contour-integral into two parts, and replacing Hankel functions with their asymptotic expressions for small 
arguments for the region between $s=-\alpha$ and $s=\alpha$ we will have:

$$
\begin{aligned}
\hat{s}_{i} \cdot \vec{H}_{i}= & \frac{k^{2}}{2 \omega \mu} \oint_{C}\left(\hat{s}_{i} \cdot \hat{s}\right) M^{\text {wall }} H_{0}^{(2)}(k \rho) d s+\frac{k}{2 \omega \mu} \oint_{C}\left(\hat{s}_{i} \cdot \hat{\rho}\right) \frac{\partial M^{\text {wall }}}{\partial s} H_{1}^{(2)}(k \rho) d s+ \\
& +\frac{k}{2 j} \oint_{C}\left(\hat{s}_{i} \cdot \hat{\varphi}\right)(\hat{s} \cdot \vec{H}) H_{1}^{(2)}(k \rho) d s
\end{aligned}
$$

where $\vec{H}_{i}=\vec{H}\left(P^{\prime}\right)$. By the same line of argument that was made to convert 2.63 into 2.65, 2.66 can be recast into the following more useful form:

$$
\begin{aligned}
\hat{s}_{i} \cdot \vec{H}_{i}= & \frac{k^{2}}{2 \omega \mu} \oint_{C}\left(\hat{s}_{i} \cdot \hat{s}\right) M^{\text {wall }} H_{0}^{(2)}(k \rho) d s-\frac{k}{2 \omega \mu} \oint_{C} M^{\text {wall }} \frac{\partial}{\partial s}\left[\left(\hat{s}_{i} \cdot \hat{\rho}\right) H_{1}^{(2)}(k \rho)\right] d s+ \\
& +\frac{k}{2 j} \oint_{C}\left(\hat{s}_{i} \cdot \hat{\varphi}\right)(\hat{s} \cdot \vec{H}) H_{1}^{(2)}(k \rho) d s
\end{aligned}
$$

which is an expression for the tangential component of magnetic field on the boundary in terms of tangential components of electric and magnetic fields on $C$. This is the new contour integral equation that has been derived for the first time in this thesis. While the conventional contour integral equation is helpful for ordinary planar circuits, it is of limited use in planar circuits containing ground plane slots. As will be seen in the next chapter, in expanding MNM to multi-layer planar structures we will need the magnetic field at arbitrary points inside the circuit periphery in terms of the tangential components of both electric and magnetic fields on the boundary. Obviously, the conventional form cannot be used for this purpose and the novel contour integral equation derived here must be used instead.

\subsubsection{Discretization of Contour-Integral Equations}

Numerical solution of contour-integral equations in 2.57 and 2.67 requires them to be discretized and cast into matrix form. If the periphery of the circuit is divided into $N^{E}$ smaller segments, having widths $w_{i}, i=1, \cdots, N^{E}$, and if each of the coupling ports is assumed to occupy one of these sections we will have [36]:

$$
\sum_{j=1}^{N^{E}} \mathbf{U}_{i j}^{E} \bar{V}_{j}^{E}=\sum_{j=1}^{N^{E}} \mathbf{H}_{i j}^{E} \bar{I}_{j}^{E} \quad i=1, \cdots, N^{E}
$$




\section{CHAPTER 2. MULTIPORT NETWORK MODEL}

where $\mathbf{U}_{i j}^{E}$ 's and $\mathbf{H}_{i j}^{E}$ 's are elements of two $N^{E} \times N^{E}$ matrices and are given as [36]:

$$
\begin{gathered}
\mathbf{U}_{i j}^{E}=\delta_{i j}-\frac{k}{2 j} \int_{w_{j}} \cos \theta H_{1}^{(2)}(k \rho) d s \\
\mathbf{H}_{i j}^{E}= \begin{cases}\frac{\omega \mu d}{2} \frac{1}{w_{j}} \int_{w_{j}} H_{0}^{(2)}(k \rho) d s & i \neq j \\
\frac{\omega \mu d}{2}\left[1-\frac{2 j}{\pi}\left(\ln \frac{k w_{i}}{4}-1+\gamma\right)\right] & i=j\end{cases}
\end{gathered}
$$

$\delta_{i j}$ is Kronecker's delta function defined in 2.23. $\gamma=0.5772 \ldots$ is Euler's constant. Note that sometimes the value of Euler's constant is given as $e^{0.5772}=1.781$ [41]. The terminal voltage and current for each coupling port are assumed to be constant over the width of the port. Therefore from 2.10 we have:

$$
\begin{aligned}
\bar{V}_{j}^{E} & \equiv d E_{z}\left(x_{j}^{s}, y_{j}^{s}\right) \\
\bar{I}_{j}^{E} & \equiv w_{j} J^{E}\left(x_{j}^{s}, y_{j}^{s}\right)
\end{aligned}
$$

where $\left(x_{j}^{s}, y_{j}^{s}\right)$ is the coordinates of the mid-point of $j^{\text {th }}$ segment (Fig. 2.6b). If $\bar{V}^{E}$ and $\bar{I}^{E}$ denote column vectors consisting of $\bar{V}_{j}^{E}$ 's and $\bar{I}_{j}^{E}$ 's, respectively, equation 2.68 can be solved for $\bar{V}^{E}$ in terms of $\bar{I}^{E}$ to give the impedance matrix of the multiport equivalent network as:

$$
\bar{V}^{E}=\mathbf{Z}^{E} \bar{I}^{E}
$$

where $\mathbf{Z}^{E}=\left(\mathbf{U}^{E}\right)^{-1} \mathbf{H}^{E} . \quad \mathbf{Z}^{E}$ is an $N^{E} \times N^{E}$ matrix and assumes that all of the segments upon the periphery are connected to coupling ports. If a particular element of $\mathbf{Z}^{E}$ is needed, it can be found from [36]:

$$
\mathbf{Z}_{i j}^{E}=\frac{1}{\operatorname{det} \mathbf{U}^{E}}\left|\begin{array}{ccccc}
\mathbf{U}_{11}^{E} & \cdots & \mathbf{H}_{1 j}^{E} & \cdots & \mathbf{U}_{1 N^{E}}^{E} \\
\vdots & & \vdots & & \vdots \\
\mathbf{U}_{N^{E} 1}^{E} & \cdots & \mathbf{H}_{N^{E} j}^{E} & \cdots & \mathbf{U}_{N^{E} N^{E}}^{E}
\end{array}\right|
$$

where $\vee^{i}$ indicates substitution into the $i^{\text {th }}$ column. Interestingly, the generalized admittance matrix cannot be calculated from 2.69 and 2.70. The diagonal elements of $\mathbf{H}^{E}$ are much smaller than non-diagonal elements. This is especially true in the close vicinity of the resonance frequencies. Consequently, in most cases, $\mathbf{H}^{E}$ is very ill-conditioned and cannot be inverted. As will be shown shortly, the new 


\section{CHAPTER 2. MULTIPORT NETWORK MODEL}

contour integral equation can be used whenever the generalized admittance matrix is needed.

Equation 2.67 can also be discretized accordingly to give:

$$
\sum_{j=1}^{N^{E}} \mathbf{A}_{i j}^{E}\left(\hat{s}_{j} \cdot \vec{H}_{j}\right)=\sum_{j=1}^{N^{E}}\left(\mathbf{B}_{i j}^{E}-\mathbf{C}_{i j}^{E}\right) \bar{M}_{j}^{\text {wall }} i=1, \cdots, N^{E}
$$

or in matrix representation:

$$
\mathbf{A}^{E}\left[\hat{s}_{j} \cdot \vec{H}_{j}\right]=\left(\mathbf{B}^{E}-\mathbf{C}^{E}\right) \bar{M}^{\text {wall }}
$$

where $\bar{M}_{j}^{\text {wall }}$ and $\hat{s}_{j} \cdot \vec{H}_{j}$ are tangential components of electric and magnetic field, respectively, at the mid-point of the $j^{\text {th }}$ segment; $\left[\hat{s}_{j} \cdot \vec{H}_{j}\right]$ and $\bar{M}^{\text {wall }}$ are column vectors consisting of $\hat{s}_{j} \cdot \vec{H}_{j}$ 's and $\bar{M}_{j}^{\text {wall }}$ 's, respectively. $\mathbf{A}^{E}, \mathbf{B}^{E}$, and $\mathbf{C}^{E}$ are $N^{E} \times N^{E}$ matrices and can be given as:

$$
\begin{aligned}
& \mathbf{A}_{i j}^{E}=\delta_{i j}-\frac{k}{2 j} \int_{w_{j}}\left(\hat{s}_{i} \cdot \hat{\varphi}\right) H_{1}^{(2)}(k \rho) d s \\
& \mathbf{B}_{i j}^{E}=\frac{\omega \varepsilon}{2}\left(\hat{s}_{i} \cdot \hat{s}_{j}\right) \int_{w_{j}} H_{0}^{(2)}(k \rho) d s \\
& \mathbf{C}_{i j}^{E}= \begin{cases}\frac{k}{2 \omega \mu}\left[\left(\hat{s}_{i} \cdot \hat{\rho}_{i, j+1}\right) H_{1}^{(2)}\left(k \rho_{i, j+1}\right)-\left(\hat{s}_{i} \cdot \hat{\rho}_{i j}\right) H_{1}^{(2)}\left(k \rho_{i j}\right)\right] & i \neq j \\
\frac{k}{\omega \mu} H_{1}^{(2)}\left(\frac{k w_{i}}{2}\right) & i=j \\
(2.76 \mathrm{c})\end{cases}
\end{aligned}
$$

where $\hat{\varphi}=\hat{z} \times \hat{\rho}$ and $\hat{\rho}$ is defined in Fig. 2.5b. We also have:

$$
\begin{aligned}
& \rho_{i j}=\sqrt{\left(x_{j}-x_{i}^{s}\right)^{2}+\left(y_{j}-y_{i}^{s}\right)^{2}} \\
& \hat{\rho}_{i j}=\hat{x} \frac{x_{j}-x_{i}^{s}}{\rho_{i j}}+\hat{y} \frac{y_{j}-y_{i}^{s}}{\rho_{i j}}
\end{aligned}
$$

It can be shown that 2.75 is in fact the inverse of 2.72. From 2.9, 2.53, and 2.71b it can be seen that $\bar{I}_{j}^{E}=w_{j}\left(\hat{s}_{j} \cdot \vec{H}_{j}\right)$. On the other hand, by looking at the definition of $\bar{M}^{\text {wall }}$, one can see that $\bar{M}_{i}^{\text {wall }}=\bar{V}_{i}^{E} / d$. Hence, by combining these with 2.72 we will have:

$$
\bar{M}_{i}^{\text {wall }}=\frac{1}{d} \sum_{j=1}^{N^{E}} \mathbf{Z}_{i j}^{E} w_{j}\left(\hat{s}_{j} \cdot \vec{H}_{j}\right)
$$




\section{CHAPTER 2. MULTIPORT NETWORK MODEL}

Numerically, this has significant importance. A look at 2.69 and 2.70 reveals that diagonal elements of $\mathbf{U}^{E}$ are all unity and larger than off-diagonal elements, while diagonal elements of $\mathbf{H}^{E}$ can be negligibly small compared to other elements of $\mathbf{H}^{E}$. The same discussion applies to $\mathbf{A}^{E}$ and $\mathbf{B}^{E}-\mathbf{C}^{E}$. Therefore, while matrices $\mathbf{U}^{E}$ and $\mathbf{A}^{E}$ are very well-conditioned, matrices $\mathbf{H}^{E}$ and $\mathbf{B}^{E}-\mathbf{C}^{E}$ can be quite ill-conditioned, meaning that 2.72 (or 2.78) can only be used to express $\bar{M}^{\text {wall }}$ in terms of $\left[\hat{s}_{j} \cdot \vec{H}_{j}\right]$, but not vice versa. On the other hand, 2.75 can only be used to express $\left[\hat{s}_{j} \cdot \vec{H}_{j}\right]$ in terms of $\bar{M}^{\text {wall }}$. In other words, with the new contour-integral equation the inverse of $\mathbf{Z}^{E}$ can be calculated analytically.

Equation 2.63 will also be needed to find the magnetic field at arbitrary points inside the contour $C$. The same steps are followed to discretize 2.63. For an arbitrary $\hat{c}$ we have:

$$
\begin{aligned}
\hat{c} \cdot \vec{H}\left(\vec{\rho}^{\prime}\right)= & \frac{k^{2}}{4 \omega \mu} \sum_{j=1}^{N^{E}}\left(\hat{c} \cdot \hat{s}_{j}\right) \bar{M}_{j}^{\text {wall }} \int_{w_{j}} H_{0}^{(2)}(k \rho) d s+\frac{k}{4 j} \sum_{j=1}^{N^{E}}\left(\hat{s}_{j} \cdot \vec{H}_{j}\right) \int_{w_{j}}(\hat{c} \cdot \hat{\varphi}) H_{1}^{(2)}(k \rho) d s+ \\
& -\frac{k}{4 \omega \mu} \sum_{j=1}^{N^{E}} \bar{M}_{j}^{\text {wall }}\left[\hat{c} \cdot \hat{\rho}_{j+1} H_{1}^{(2)}\left(k \rho_{j+1}\right)-\hat{c} \cdot \hat{\rho}_{j} H_{1}^{(2)}\left(k \rho_{j}\right)\right]
\end{aligned}
$$

where $\rho$ and $\hat{\rho}$ have the same definitions as in Fig. 2.5 except for the fact that the point $\left(x_{n}^{s}, y_{n}^{s}\right)$ is replaced by the point $\left(x_{0}, y_{0}\right)$, the coordinates of $\vec{\rho}=\vec{\rho}^{\prime}$. We also have $\hat{\varphi}=\hat{z} \times \hat{\rho}$ and:

$$
\begin{aligned}
& \rho_{j}=\sqrt{\left(x_{j}-x_{0}\right)^{2}+\left(y_{j}-y_{0}\right)^{2}} \\
& \hat{\rho}_{j}=\hat{x} \frac{x_{j}-x_{0}}{\rho_{j}}+\hat{y} \frac{y_{j}-y_{0}}{\rho_{j}}
\end{aligned}
$$




\section{Chapter 3}

\section{Extension of 2D Multiport Network Model to Planar Circuits with Ground Plane Slots}

Slots in ground planes have become very common in multi-layer circuits and antennas. The existing conventional 2D circuit approach proposed by Okoshi [36], [42] and its later refinements cannot include any slots in ground plane as it violates the fundamental assumption that only $T M^{z}$ modes are excited. In this chapter, we will combine MNM method with a generalized network formulation for aperture problems to extend the applicability of network model to multi-layer planar circuits.

\subsection{Generalized Network Formulation for Aperture Problems}

Coupling through apertures has many applications in microwave engineering. Apertures in conducting screens, such as waveguide-fed apertures, cavity-fed apertures, waveguide-to-waveguide coupling, waveguide-to-cavity coupling, and cavity-to-cavity coupling, a few to name, has been extensively addressed in the literature. The interested reader can consult [41], [51], or [52] for a classic treatment of the problem and further references. However, the formulation of aperture coupling in terms of 


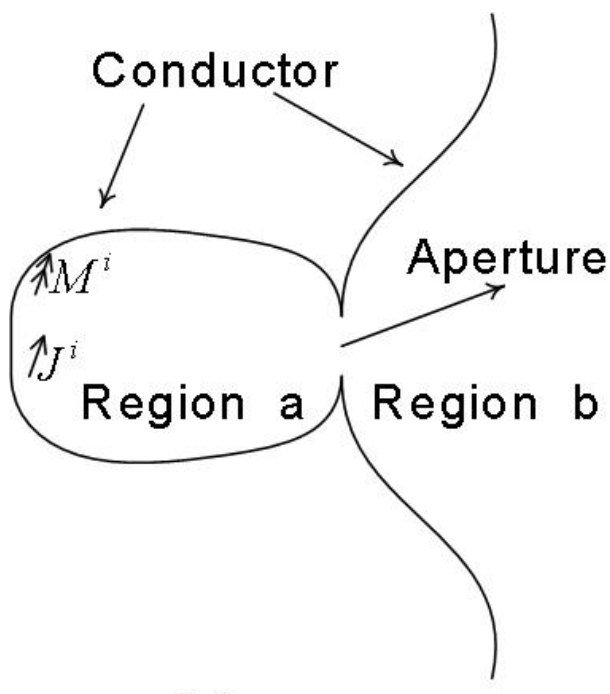

(a)

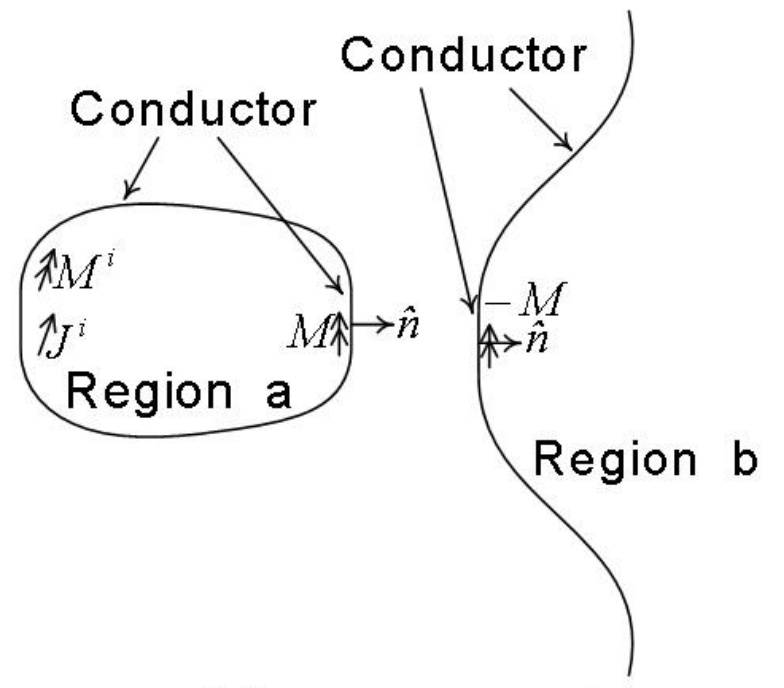

(b)

(c)

Figure 3.1: General problem of aperture coupling [53], a) original problem, b) equivalent problem for region a, c) equivalent problem for region $\mathrm{b}$

low-frequency network parameters by Harrington et al. [53] is best suited for our purpose and will be introduced here.

The general problem of aperture coupling between two regions is shown in Fig. 3.1a. These two regions are designated as region $\mathbf{a}$ and region $\mathbf{b}$, being coupled only through the aperture and isolated otherwise. Here, it has been assumed that each region is bounded by a perfect electric conductor. However, there is no restriction on the type of the boundary as far as the two regions are isolated except for coupling through the aperture. It is also worth mentioning that both regions can be closed or they can both open to infinity. Region a is assumed to contain impressed sources denoted by $J^{i}$ and $M^{i}$, while, without loss of generality, region $\mathbf{b}$ is assumed to be source free. If both regions contain impressed sources, the problem can be regarded as the superposition of two separate problems with sources in only one of the regions.

The problem can be divided into two equivalent problems, shown in Fig. 3.1b and Fig. 3.1b, one for each region, by using the surface equivalence theorem to cover the slot by a perfect electric conductor with equivalent surface magnetic currents on each side [41]. Let $\vec{E}^{a}$ represent total electric field in region a, due to both impressed and equivalent sources. In order for $\vec{E}^{a}$ to satisfy boundary conditions, 


\section{CHAPTER 3. EXTENSION OF 2D MULTIPORT NETWORK MODEL TO ...}

we must have:

$$
\vec{M}^{\text {slot }}=\hat{n} \times \vec{E}^{a} \quad \text { over } S_{a}
$$

where $S_{a}$ denotes aperture surface. Region b does not contain any impressed sources and fields in that region will only be produced by coupling from region a or equivalently by an equivalent surface magnetic current over the closed aperture. Continuity of tangential electric field over the aperture requires that the equivalent surface magnetic current in this region be $-\vec{M}^{\text {slot }}$. The remaining boundary condition, i.e. continuity of the tangential magnetic field over the aperture, must be enforced explicitly. Let $\vec{H}_{t}^{a}$ denote the total tangential magnetic field due to both impressed and equivalent sources in region a. $\vec{H}_{t}^{a}$ will be composed of two terms as:

$$
\vec{H}_{t}^{a}=\vec{H}_{t}^{i}+\vec{H}_{t}^{a}\left(\vec{M}^{s l o t}\right)
$$

where $\vec{H}_{t}^{i}$ and $\vec{H}_{t}^{a}\left(\vec{M}^{\text {slot }}\right)$ are tangential components of magnetic field due to the impressed and equivalent sources, respectively, with the aperture closed. In region b the total tangential magnetic field is only due to $-\vec{M}^{\text {slot }}$, with the aperture closed. Let $\vec{H}_{t}^{b}\left(-\vec{M}^{\text {slot }}\right)$ denote tangential magnetic field over the aperture in region $\mathbf{b}$. Continuity of tangential magnetic filed across the aperture requires that:

$$
\vec{H}_{t}^{a}\left(\vec{M}^{s l o t}\right)+\vec{H}_{t}^{b}\left(\vec{M}^{s l o t}\right)=-\vec{H}_{t}^{i}
$$

where use has been made of the fact that because of the linearity of Maxwell's equations we have $\vec{H}_{t}^{b}\left(-\vec{M}^{\text {slot }}\right)=-\vec{H}_{t}^{b}\left(\vec{M}^{\text {slot }}\right)$. Equation 3.83 is the basic operator equation for determining the equivalent surface magnetic current $\vec{M}^{\text {slot }}$. To this end, a set of $N^{S}$ expansion or basis functions are chosen to expand $\vec{M}^{\text {slot }}$ as follows [54]:

$$
\vec{M}^{s l o t}=\sum_{\ell} \bar{V}_{\ell}^{S} \vec{M}_{\ell}
$$

where $\bar{V}_{\ell}^{S}$ are unknown coefficients to be determined by method of moments. By substituting 3.84 into 3.83 and using linearity of Maxwell's equations, we will have:

$$
\sum_{\ell} \bar{V}_{\ell}^{S} \vec{H}_{t}^{a}\left(\vec{M}_{\ell}\right)+\sum_{\ell} \bar{V}_{\ell}^{S} \vec{H}_{t}^{b}\left(\vec{M}_{\ell}\right)=-\vec{H}_{t}^{i}
$$

Equation 3.85 cannot be solved exactly for the unknown coefficients, as it would require an infinite number of basis functions in most cases. Instead, it will be solved approximately on an average sense by testing both sides with testing functions. To accomplish this, a set of $N^{S}$ testing functions (the same number as basis functions) is chosen, which may or may not be equal to the basis functions. Let $W_{n}$ 's, where 
$n=1, \cdots, N^{S}$ represent these testing functions. We also define a symmetric inner product of two vectors in the linear space spanned by $\vec{M}_{\ell}$ 's as:

$$
\langle\vec{A}, \vec{B}\rangle=\iint_{S_{a}} \vec{A} \cdot \vec{B} d S
$$

By testing both sides of 3.85 with testing functions, we obtain a set of $N^{S}$ linear equations for $N^{S}$ unknown coefficients as [53]:

$$
\sum_{\ell} \bar{V}_{\ell}^{S}\left\langle\vec{H}_{t}^{a}\left(\vec{M}_{\ell}\right), \vec{W}_{n}\right\rangle+\sum_{\ell} \bar{V}_{\ell}^{S}\left\langle\vec{H}_{t}^{b}\left(\vec{M}_{\ell}\right), \vec{W}_{n}\right\rangle=-\left\langle\vec{H}_{t}^{i}, \vec{W}_{n}\right\rangle
$$

for $n=1, \cdots, N^{S}$. This system of $N^{S}$ linear equations can be solved to determine the unknown coefficients, i.e. $\bar{V}_{\ell}^{S}$ 's, and consequently the equivalent surface magnetic current over the aperture. Once $\vec{M}^{\text {slot }}$ has been determined, the fields and field related parameters can be found. Equation 3.87 can be put into matrix form by defining an admittance matrix for each region as:

$$
\begin{aligned}
Y_{n \ell}^{a} & =-\left\langle\vec{H}_{t}^{a}\left(\vec{M}_{\ell}\right), \vec{W}_{n}\right\rangle \\
Y_{n \ell}^{b} & =-\left\langle\vec{H}_{t}^{b}\left(\vec{M}_{\ell}\right), \vec{W}_{n}\right\rangle
\end{aligned}
$$

where $Y^{a}$ and $Y^{b}$ are $N^{S} \times N^{S}$ admittance matrices for regions a and $\mathbf{b}$, respectively. The original paper by Harrington [53] places minus signs in 3.88a and 3.88b on the basis of power considerations. This will be clarified later. A source vector can also be defined as:

$$
\bar{I}^{i}=\left\langle\vec{H}_{t}^{i}, \vec{W}_{n}\right\rangle
$$

Therefore, 3.87 can be cast into the following matrix form:

$$
\left[Y^{a}+Y^{b}\right] \bar{V}^{S}=\bar{I}^{i}
$$

where $\bar{V}^{S}$ is the vector of coefficients. Equation 3.90 can be interpreted in terms of generalized networks as two networks with admittance matrices $Y^{a}$ and $Y^{b}$ in parallel with the current source $\bar{I}^{i}$ (Fig. 3.2).

An important observation can be made about $Y^{a}$ and $Y^{b} ; Y^{a}$ involves only region a and $Y^{b}$ involves only region $\mathbf{b}$. Hence the admittance matrix computed for one region can be combined with that of any other region as far as the coupling is through the same aperture and the same basis and testing functions are used in both problems. For example, the same aperture admittance matrix used for radiation into half-space would be equally useful for cavity excitation. In other words, one can solve a few canonical problems and then use it in various other aperture-coupling problems. 
CHAPTER 3. EXTENSION OF 2D MULTIPORT NETWORK MODEL TO ...

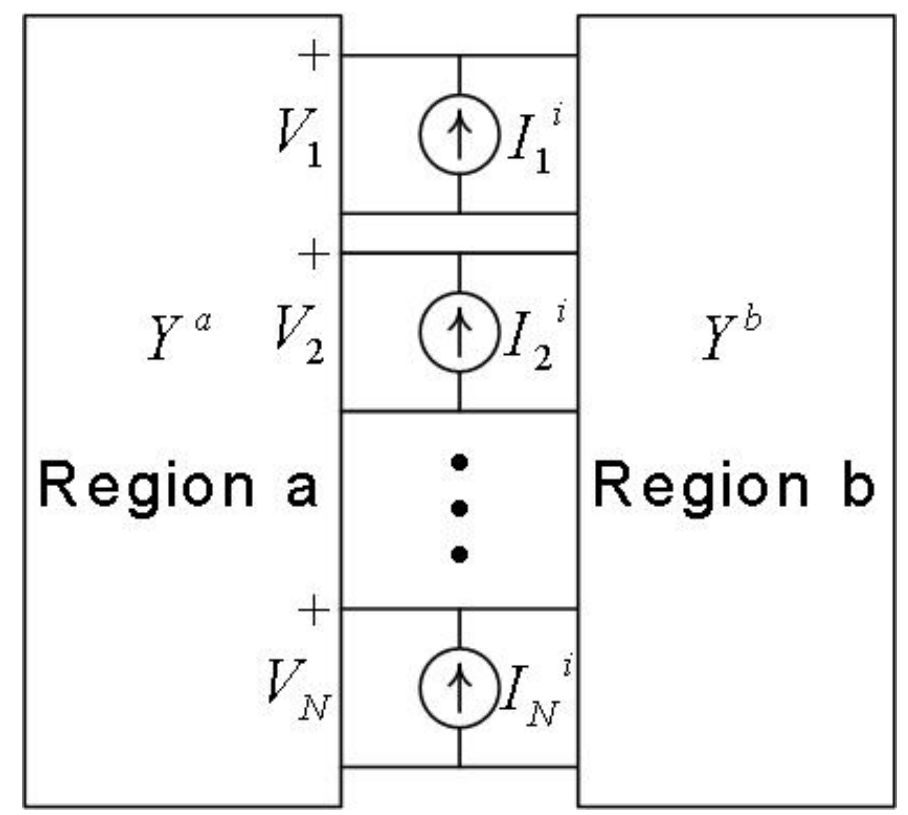

Figure 3.2: Generalized network interpretation of 3.90

\subsubsection{Transmitted Power Considerations}

The complex power transmitted through the aperture from region a into region b can be written as:

$$
P_{t}=\frac{1}{2} \iint_{S_{a}}\left(\vec{E}^{a} \times \vec{H}^{a *}\right) \cdot \hat{n} d S
$$

where the asterisk denotes complex conjugate. By substituting from 3.81, 3.91 can be written as:

$$
P_{t}=\frac{1}{2} \iint_{S_{a}} \vec{M}^{s l o t} \cdot \vec{H}^{a *} d S
$$

Since $\vec{M}^{\text {slot }}$ only has component tangent to the aperture, the inner product in 3.92 involves only the tangential component of the magnetic field over the aperture. 
From $3.82,3.83$, and 3.84 we have:

$$
\begin{aligned}
P_{t} & =\frac{1}{2} \iint_{S_{a}} \vec{M}^{s l o t} \cdot \vec{H}_{t}^{b *}\left(-\vec{M}^{s l o t}\right) d S \\
& =-\frac{1}{2} \sum_{n} \sum_{\ell} \bar{V}_{n}^{S} \bar{V}_{\ell}^{S *} \iint_{S_{a}} \vec{M}_{n} \cdot \vec{H}_{t}^{b *}\left(\vec{M}_{\ell}\right) d S
\end{aligned}
$$

If testing functions are chosen to be the same as basis functions (Galerkin's method), i.e. $W_{n}=M_{n}$, and if basis functions are all real, then from $3.88 \mathrm{~b}$ it can be seen that:

$$
\begin{aligned}
P_{t} & =\frac{1}{2} \sum_{n} \sum_{\ell} \bar{V}_{n}^{S} \bar{V}_{\ell}^{S *} Y_{n \ell}^{b *} \\
& =\frac{1}{2} \bar{V}^{S} Y^{b *} \bar{V}^{S *}
\end{aligned}
$$

which is a familiar formula from network theory as the power into the network with admittance matrix $Y^{b}$. It also justifies the minus sign in 3.88a and 3.88b. However, to avoid confusion, we have dropped the minus signs in front of the admittance matrices.

\subsection{Hybrid Matrix Formulation}

We consider the problem of a microstrip patch on a slotted ground plane, as illustrated in Fig. 3.3. The coordinate system is chosen in a way that the metallic patch lies in $x y$-plane. The ground plane coincides with the plane $z=-d$ parallel to the $x y$-plane. As it was explained in previous chapter, in MNM for open-boundary planar circuits, the periphery of the planar structure is divided up into sufficient number of smaller segments and each segment is treated as a port in low-frequency circuits having a terminal voltage and current [36]. The width of each port is taken to be much smaller than the wavelength to make sure that the current density is almost constant over the width of each port and consequently a port current can be defined as the product of this current density and the width of the port. Each port has a self-impedance and mutual-impedances with the other ports which account for interaction between voltages and currents of corresponding ports. The whole circuit is represented by an impedance-matrix, which relates ports voltages and 
currents. We will term these ports as edge ports and will use the superscript $E$ to distinguish them from slot ports, which will be defined later.

In the absence of any slots, the MNM for an open-boundary planar circuit can be simply given by 2.72 . If the planar circuit is slot-coupled to another planar circuit on the other side of a common ground plane, there will be scattered fields from the slot that will modify field patterns on both sides. The surface equivalence theorem is used to replace the slot with an equivalent surface magnetic current dented by $\vec{M}^{\text {slot }}$. Consequently, there will also be voltage induced at the edge ports due to $\vec{M}^{\text {slot}}$, which accounts for the presence of scattered fields. Therefore, 2.72 can be modified as follows:

$$
\bar{V}^{E}=\mathbf{Z}^{E} \bar{I}^{E}+\bar{V}^{E}\left(\vec{M}^{\text {slot }}\right)
$$

where $V^{E}\left(\vec{M}^{\text {slot }}\right)=d E_{z}\left(\vec{M}^{\text {slot }}\right)$ and $E_{z}\left(\vec{M}^{\text {slot }}\right)$ are the induced voltage and $z$ directed electric field due to $\vec{M}^{\text {slot }}$ at edge ports, respectively. In particular, for the $m^{\text {th }}$ edge port we will have:

$$
\bar{V}_{m}^{E}=\sum_{m=1}^{N^{E}} \mathbf{Z}_{m n}^{E} \bar{I}_{n}^{E}+\bar{V}_{m}^{E}\left(\vec{M}^{s l o t}\right) \quad m=1, \cdots, N^{E}
$$

where as before $N^{E}$ is the number of segments along the periphery of the circuit and $\bar{V}_{m}^{E}\left(\vec{M}^{\text {slot }}\right)$ is the induced voltage at the $m^{\text {th }}$ edge port due to $\vec{M}^{\text {slot }}$. Since $\vec{M}^{\text {slot }}$ is unknown, a set of basis functions will be used to expand $\vec{M}^{\text {slot }}$ as:

$$
\vec{M}^{s l o t}=\sum_{\ell=1}^{N^{S}} \bar{V}_{\ell}^{S} \vec{M}_{\ell}
$$

where $\bar{V}_{\ell}^{S}$ 's and $\vec{M}_{\ell}$ 's are expansion coefficients and basis functions, respectively. $N^{S}$ is the number of basis functions used to expand the equivalent magnetic current over the slot. Due to linearity of Maxwell's equations, we will have:

$$
\bar{V}_{m}^{E}=\sum_{m=1}^{N^{E}} \mathbf{Z}_{m n}^{E} \bar{I}_{n}^{E}+\sum_{\ell=1}^{N^{S}} \bar{V}_{\ell}^{S} \bar{V}_{m}^{E}\left(\vec{M}_{\ell}\right) \quad m=1, \cdots, N^{E}
$$

In order to derive an impedance matrix representation for the two-layer circuit, a similar equation for the planar circuit below the ground plane is written and linked to 3.98. This can be accomplished by enforcing electromagnetic boundary conditions over the slot. Similar to the approach taken in [53], continuity of the electric field is ensured if the equivalent magnetic currents on the two sides of the 


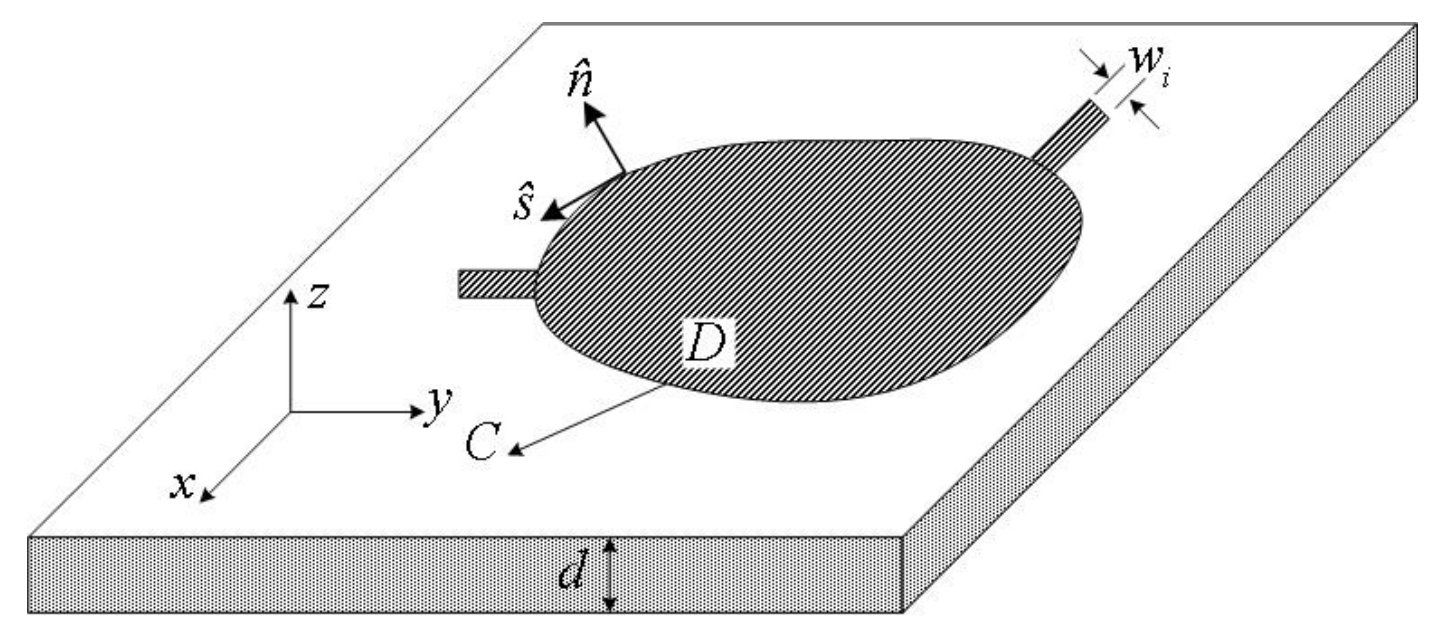

(a)

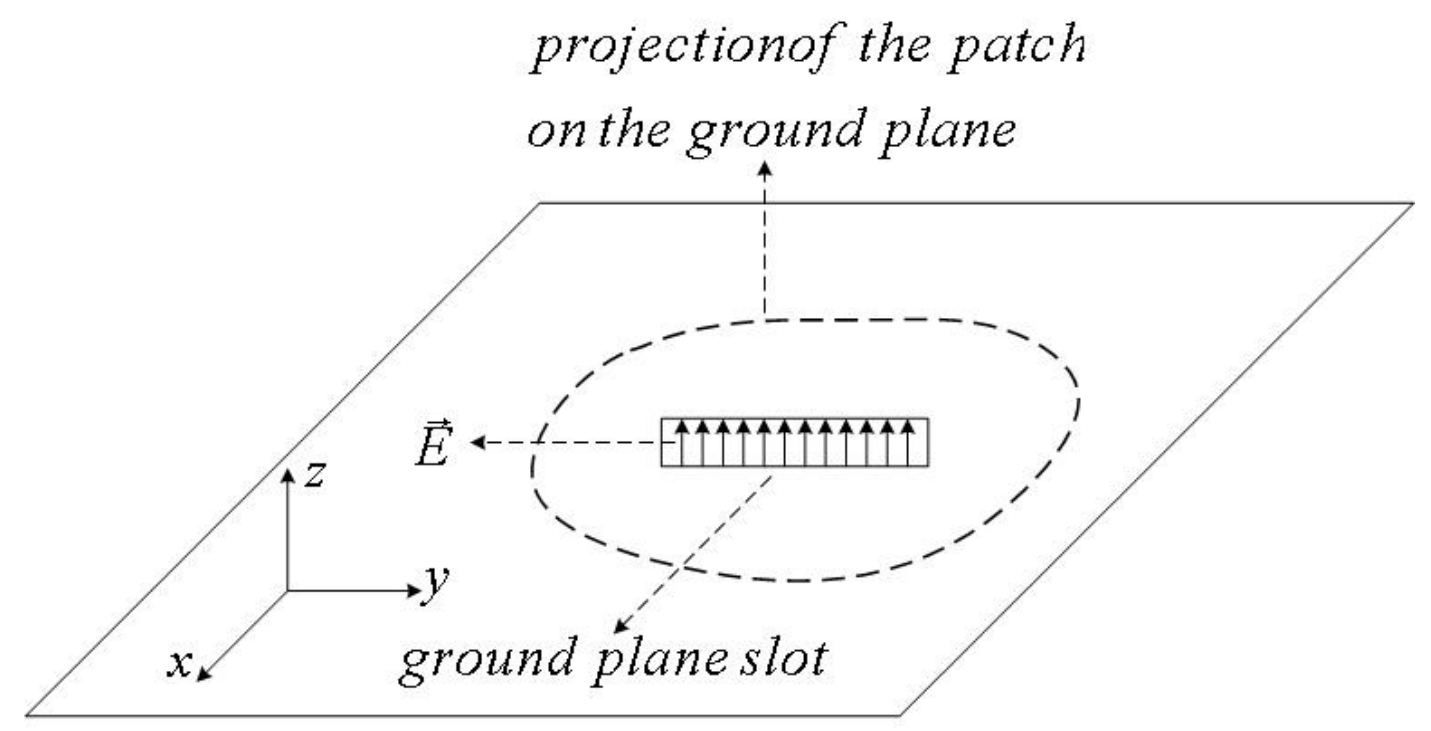

(b)

Figure 3.3: a) An irregularly shaped patch on a ground plane with a slot and b) Projection of the patch on the ground plane 
slot are of the same magnitude but opposite signs. Continuity of the magnetic field, however, must be explicitly enforced. Here we take an approach similar to the one taken in the generalized network formulation for aperture problems [53]. Input currents at the edge ports, which can be viewed as impressed sources, and the equivalent magnetic current over the slot both contribute to the tangential magnetic field on the slot. This can be written as:

$$
\vec{H}_{T}^{t o t}=\sum_{n=1}^{N^{E}} \vec{H}_{T}\left(\bar{I}_{n}^{E}\right)+\sum_{\ell=1}^{N^{S}} \bar{V}_{\ell}^{S} \vec{H}_{T}\left(\vec{M}_{\ell}\right)
$$

where $\vec{H}_{T}\left(\bar{I}_{n}^{E}\right)$ and $\vec{H}_{T}\left(\vec{M}_{\ell}\right)$ are transverse components of magnetic field due to $\bar{I}_{n}^{E}$ and $\vec{M}_{\ell}$ over the slot, respectively. This is the basic operator equation for the unknown equivalent magnetic current over the slot. Following the same procedure, we test both sides of 3.99 with $N^{S}$ vector testing functions according to the definition given in 3.86 for the inner product of two vectors in the linear space spanned by $\vec{M}_{\ell}$ 's. Consequently we will have:

$$
\left\langle\vec{H}_{T}^{t o t}, \vec{W}_{m}\right\rangle=\sum_{n=1}^{N^{E}}\left\langle\vec{H}_{T}\left(\bar{I}_{n}^{E}\right), \vec{W}_{m}\right\rangle+\sum_{\ell=1}^{N^{S}} \bar{V}_{\ell}^{S}\left\langle\vec{H}_{T}\left(\vec{M}_{\ell}\right), \vec{W}_{m}\right\rangle m=1, \cdots, N^{S}
$$

where $\vec{W}_{m}$ 's are testing functions. If we think of $\bar{V}_{\ell}^{S}$ 's as slot port voltages and define slot port currents as $\left\langle\vec{H}_{T}^{\text {tot }}, \vec{W}_{m}\right\rangle$, we will have a hybrid matrix representation for a planar circuit with a slot in ground plane as follows:

$$
\left\{\begin{array}{l}
\bar{V}^{E}=\mathbf{Z}^{E} \bar{I}^{E}+\mathbf{H}^{E S} \bar{V}^{S} \\
\bar{I}^{S}=\mathbf{H}^{S E} \bar{I}^{E}+\mathbf{Y}^{S} \bar{V}^{S}
\end{array}\right.
$$

where:

$$
\begin{aligned}
& \mathbf{H}_{m n}^{E S}=\bar{V}_{m}^{E}\left(\vec{M}_{n}\right) \\
& \mathbf{H}_{m n}^{S E}=\left\langle\vec{H}_{T}\left(\bar{I}_{n}^{E}\right), \vec{W}_{m}\right\rangle / \bar{I}_{n}^{E} \\
& \mathbf{Y}_{m n}^{S}=\left\langle\vec{H}_{T}\left(\vec{M}_{n}\right), \vec{W}_{m}\right\rangle
\end{aligned}
$$

$\mathbf{H}_{m n}^{E S}$ is the voltage induced at the $m^{\text {th }}$ edge port due to $\vec{M}_{n}$ and will be referred to as "Slot-to-Edge Transfer Matrix". $\mathbf{H}_{m n}^{S E}$ is the transverse component of the magnetic field due to $\bar{I}_{n}^{E}=1$ tested by $\vec{W}_{m}$ and is called "Slot-to-Edge Transfer Matrix". $\mathbf{Y}^{S}$ is the "Slot Admittance Matrix" and accounts for the interaction of slot ports 
together. $\mathbf{Y}_{m n}^{S}$ is in fact the transverse component of the magnetic field due to $\vec{M}_{n}$ tested by $\vec{W}_{m}$. This is also similar to the generalized admittance matrices defined in $3.88 \mathrm{~b}$ and $3.88 \mathrm{a}$.

It will be shown that if testing functions are the same as basis functions, $\mathbf{H}^{E S}$ and $\mathbf{H}^{S E}$ are transpose matrices. It is worth mentioning that there is no restriction on the size or shape of the slot.

\subsection{Calculation of Impedance Matrix in terms of Hybrid Sub-Matrices}

Once the hybrid sub-matrices for the planar circuits above and below the ground plane are evaluated, the impedance matrix of the two-layer structure can be readily obtained by simple algebra. We will use subscripts $\ell$ and $u$ to distinguish the two planar circuits. From previous section we have:

$$
\begin{array}{r}
\left\{\begin{array}{l}
\bar{V}_{u}^{E}=\mathbf{Z}_{u}^{E} \bar{I}_{u}^{E}+\mathbf{H}_{u}^{E S} \bar{V}_{u}^{S} \\
\bar{I}_{u}^{S}=\mathbf{H}_{u}^{S E} \bar{I}_{u}^{E}+\mathbf{Y}_{u}^{S} \bar{V}_{u}^{S}
\end{array}\right. \\
\left\{\begin{array}{c}
\bar{V}_{\ell}^{E}=\mathbf{Z}_{\ell}^{E} \bar{I}_{\ell}^{E}+\mathbf{H}_{\ell}^{E S} \bar{V}_{\ell}^{S} \\
\bar{I}_{\ell}^{S}=\mathbf{H}_{\ell}^{S E} \bar{I}_{\ell}^{E}+\mathbf{Y}_{\ell}^{S} \bar{V}_{\ell}^{S}
\end{array}\right.
\end{array}
$$

Continuity of the electric and magnetic fields over the slot require that $\bar{I}_{u}^{S}=\bar{I}_{\ell}^{S}=$ $\bar{I}^{S}$, and $\bar{V}_{u}^{S}=-\bar{V}_{\ell}^{S}=\bar{V}^{S}$. After eliminating $\bar{V}^{S}$ and $\bar{I}^{S}$ the impedance matrix for the two-layer structure can be written as:

$$
\left[\begin{array}{c}
\bar{V}_{u}^{E} \\
\bar{V}_{\ell}^{E}
\end{array}\right]=\left[\begin{array}{ll}
\mathbf{Z}_{u u} & \mathbf{Z}_{u \ell} \\
\mathbf{Z}_{\ell u} & \mathbf{Z}_{\ell \ell}
\end{array}\right]\left[\begin{array}{c}
\bar{I}_{u}^{E} \\
\bar{I}_{\ell}^{E}
\end{array}\right]
$$

where

$$
\begin{aligned}
\mathbf{Z}_{u u} & =\mathbf{Z}_{u}^{E}-\mathbf{H}_{u}^{E S}\left(\mathbf{Y}_{u}^{S}+\mathbf{Y}_{\ell}^{S}\right)^{-1} \mathbf{H}_{u}^{S E} \\
\mathbf{Z}_{u \ell} & =\mathbf{H}_{u}^{E S}\left(\mathbf{Y}_{u}^{S}+\mathbf{Y}_{\ell}^{S}\right)^{-1} \mathbf{H}_{\ell}^{S E} \\
\mathbf{Z}_{\ell u} & =\mathbf{H}_{\ell}^{E S}\left(\mathbf{Y}_{u}^{S}+\mathbf{Y}_{\ell}^{S}\right)^{-1} \mathbf{H}_{u}^{S E} \\
\mathbf{Z}_{\ell \ell} & =\mathbf{Z}_{\ell}^{E}-\mathbf{H}_{\ell}^{E S}\left(\mathbf{Y}_{u}^{S}+\mathbf{Y}_{\ell}^{S}\right)^{-1} \mathbf{H}_{\ell}^{S E}
\end{aligned}
$$

Note that if $\mathbf{H}_{u}^{E S}$ and $\mathbf{H}_{\ell}^{E S}$ are transpose matrices of $\mathbf{H}_{u}^{S E}$ and $\mathbf{H}_{\ell}^{S E}$, respectively, $\mathbf{Z}_{u \ell}$ and $\mathbf{Z}_{\ell u}$ will be transpose matrices as well. 


\subsection{Computation of Hybrid Sub-Matrices for Regular Shapes}

\subsection{1 $\mathrm{Z}^{E}$ : Edge Impedance Matrix}

The edge impedance matrix for regular shapes in terms of their eigenfunctions is given in 2.27. From 2.27 we have:

$$
\mathbf{Z}_{i j}^{E}=j \omega \mu d \sum_{n=1}^{\infty} \frac{\Psi_{i n} \Psi_{j n}^{*}}{k_{n}^{2}-k^{2}}
$$

where

$$
\Psi_{i n}=\frac{1}{w_{i}} \int_{w_{i}} \phi_{n}(s) d s
$$

\subsection{2 $\mathbf{H}^{E S}$ : Slot-To-Edge Transfer Matrix}

It was already discussed that for thin planar circuits the fields are assumed to be $T M^{z}$ with no $z$ variation. Let $\phi_{m}(x, y)$ 's be the normalized eigenfunctions for this regularly shaped planar circuit:

$$
E_{z}=\sum_{m=1}^{\infty} A_{m} \phi_{m}(x, y)
$$

where $A_{m}$ 's are expansion coefficients.

The transverse components of the electric field are identically zero due to the fact that there is no $z$ variation. This can be clearly seen from the following equations:

$$
E_{x}^{T M} \propto \frac{\partial^{2} E_{z}}{\partial x \partial z}, E_{y}^{T M} \propto \frac{\partial^{2} E_{z}}{\partial y \partial z}
$$

Apparently, these fields satisfy the boundary condition requiring that the tangential electric field vanish on conducting surfaces. The existence of the equivalent magnetic current over the slot dictates new boundary condition on the ground 
plane, though the boundary condition on the top plate remains the same. In order to account for the presence of the equivalent magnetic current, we propose that the following form be adopted for modal expansion in such a cavity [55], [56]:

$$
E_{z}=\sum_{m=1}^{\infty} A_{m} \phi_{m}(x, y) \cos \beta_{m}(d-z)
$$

where $\beta_{m}=\sqrt{k^{2}-k_{m}^{2}}$ and $k_{m}$ is the resonant wave number of the two dimensional cavity. From 3.110 the transverse components of electric field can be given as:

$$
\begin{aligned}
& E_{x}^{T M}=\sum_{m=1}^{\infty} \frac{A_{m} \beta_{m}}{k^{2}-\beta_{m}^{2}} \frac{\partial \phi_{m}(x, y)}{\partial x} \sin \beta_{m}(d-z) \\
& E_{y}^{T M}=\sum_{m=1}^{\infty} \frac{A_{m} \beta_{m}}{k^{2}-\beta_{m}^{2}} \frac{\partial \phi_{m}(x, y)}{\partial y} \sin \beta_{m}(d-z)
\end{aligned}
$$

While the transverse components of electric field still vanish on the top plate at $z=d$, they no longer do so on the ground plane. Therefore, the existence of the equivalent magnetic current on the slot can be taken into account as follows:

$$
\left.\vec{E}^{T M}\right|_{z=0} \times \hat{z}=\vec{M}^{s l o t}=\sum_{\ell=1}^{N^{S}} \bar{V}_{\ell}^{S} \vec{M}_{\ell}
$$

From which the expansion coefficients can be found as:

$$
A_{m}=\frac{k^{2}-\beta_{m}^{2}}{\beta_{m} F_{m}} \frac{1}{\sin \beta_{m} d} \sum_{\ell=1}^{N^{S}} \bar{V}_{\ell}^{S} L_{\ell m}
$$

where

$$
\begin{aligned}
F_{m} & =\iint_{D}\left(\left|\frac{\partial \phi_{m}(x, y)}{\partial x}\right|^{2}+\left|\frac{\partial \phi_{m}(x, y)}{\partial y}\right|^{2}\right) d S \\
L_{\ell m} & =\iint_{\text {slot }} \vec{M}_{\ell} \cdot\left(\hat{x} \frac{\partial \phi_{m}^{*}(x, y)}{\partial y}-\hat{y} \frac{\partial \phi_{m}^{*}(x, y)}{\partial x}\right) d S
\end{aligned}
$$

We have also used the fact that:

$$
\iint_{D}\left(\frac{\partial \phi_{m}(x, y)}{\partial x} \frac{\partial \phi_{n}^{*}(x, y)}{\partial x}+\frac{\partial \phi_{m}(x, y)}{\partial y} \frac{\partial \phi_{n}^{*}(x, y)}{\partial y}\right) d S=0
$$


A proof has been given in Appendix A for 3.115. Hence the voltage induced at the $j^{\text {th }}$ edge port due to the equivalent magnetic current over the slot becomes:

$$
\begin{aligned}
\bar{V}_{j}^{E}\left(\vec{M}^{\text {slot }}\right) & =\frac{1}{w_{j} d} \int_{w_{j}} \int_{0}^{d} E_{z}(s, z) d z d s \\
& =\frac{1}{d} \sum_{\ell=1}^{N^{S}} \bar{V}_{\ell}^{S} \sum_{m=1}^{\infty} \frac{k^{2}-\beta_{m}^{2}}{\beta_{m}^{2} F_{m}} L_{\ell m} \Psi_{j m}
\end{aligned}
$$

Finally we have:

$$
\mathbf{H}_{j \ell}^{E S}=\bar{V}_{j}^{E}\left(\vec{M}_{\ell}\right)=\frac{1}{d} \sum_{m=1}^{\infty} \frac{k^{2}-\beta_{m}^{2}}{\beta_{m}^{2} F_{m}} L_{\ell m} \Psi_{j m}
$$

\subsection{3 $\quad \mathrm{Y}^{S}$ : Slot Admittance Matrix}

$\mathbf{Y}^{S}$ accounts for interaction among basis functions used to expand the equivalent magnetic current over the slot. This equivalent magnetic current can excite $T E^{z}$ modes as well as $T M^{z}$ modes. For $T M^{z}$ fields we start from 3.110 to calculate the transverse components of magnetic field due to the equivalent magnetic current over the slot.

$$
\left.\vec{H}_{T}^{T M}\left(\vec{M}_{\ell}\right)\right|_{z=0}=j \omega \varepsilon \sum_{m=1}^{\infty} \frac{\cot \beta_{m} d}{\beta_{m} F_{m}} L_{\ell m}\left(\hat{x} \frac{\partial \phi_{m}(x, y)}{\partial y}-\hat{y} \frac{\partial \phi_{m}(x, y)}{\partial x}\right)
$$

Similar to $T M^{z}$ case, the following form can be proposed for $H_{z}$ of $T E^{z}$ modes:

$$
H_{z}=\frac{1}{j \omega \mu} \sum_{m=1}^{\infty} A_{m}^{\prime} \phi_{m}^{T E}(x, y) \sin \beta_{m}(d-z)
$$

from which the transverse components of electric field and consequently the expansion coefficients can be obtained as:

$$
A_{m}^{\prime}=\frac{k^{2}-\beta_{m}^{2}}{P_{m}} \frac{1}{\sin \beta_{m} d} \sum_{\ell=1}^{N^{S}} V_{\ell}^{S} R_{\ell m}
$$


where

$$
\begin{aligned}
P_{m} & =\iint_{D}\left(\left|\frac{\partial \phi_{m}^{T E}(x, y)}{\partial x}\right|^{2}+\left|\frac{\partial \phi_{m}^{T E}(x, y)}{\partial y}\right|^{2}\right) d S \\
R_{\ell m} & =\iint_{\text {slot }} \vec{M}_{\ell} \cdot\left(\hat{x} \frac{\partial \phi_{m}^{T E *}(x, y)}{\partial x}+\hat{y} \frac{\partial \phi_{m}^{T E *}(x, y)}{\partial y}\right) d S
\end{aligned}
$$

Hence, the transverse components of magnetic field for $T E^{z}$ fields become:

$$
\left.\vec{H}_{T}^{T E}\left(\vec{M}_{\ell}\right)\right|_{z=0}=\frac{-1}{j \omega \mu} \sum_{m=1}^{\infty} \frac{\beta_{m} \cot \beta_{m} d}{P_{m}} R_{\ell m}\left(\hat{x} \frac{\partial \phi_{m}^{T E}(x, y)}{\partial x}+\hat{y} \frac{\partial \phi_{m}^{T E}(x, y)}{\partial y}\right)
$$

And finally from 3.102a we will have:

$$
\begin{aligned}
\mathbf{Y}_{n \ell}^{S} & =\left\langle\left.\vec{H}_{T}^{T M}\left(\vec{M}_{\ell}\right)\right|_{z=0}, \vec{M}_{n}\right\rangle+\left\langle\left.\vec{H}_{T}^{T E}\left(\vec{M}_{\ell}\right)\right|_{z=0}, \vec{M}_{n}\right\rangle \\
& =j \omega \varepsilon \sum_{m=1}^{\infty} \frac{\cot \beta_{m} d}{\beta_{m}} \frac{L_{\ell m} L_{n m}^{*}}{F_{m}}-\frac{1}{j \omega \mu} \sum_{m=1}^{\infty} \beta_{m} \cot \beta_{m} d \frac{R_{\ell m} R_{n m}^{*}}{P_{m}}
\end{aligned}
$$

\subsection{Computation of Hybrid Sub-Matrices for Irregular Shapes}

In this section, sub-matrices in hybrid matrix representation will be derived for an arbitrarily shaped planar circuit using contour-integral equations. The symbols and notations are the same as the ones shown in Fig. 2.6 and Fig. 3.3. Briefly, $C$, the contour of the circuit periphery, is divided into $N^{E}$ segments, small enough to be approximated by a straight line. The coordinates of the dividing points are denoted by $\left(x_{n}, y_{n}\right)$ 's, $n=1, \cdots, N^{E}$, and the line segment connecting $\left(x_{n}, y_{n}\right)$ and $\left(x_{n+1}, y_{n+1}\right)$ is referred to as the $n^{t h}$ segment and will be denoted by $C_{n}$. These points are numbered in counterclockwise direction, as shown in Fig. 2.6. This is due to the fact that in deriving the contour-integral equation, distance along the contour $C$ is measured in counterclockwise direction and the tangent and outward normal to the $n^{\text {th }}$ segment, denoted by $\hat{s}_{n}$ and $\hat{n}_{n}$, respectively, are defined in a way that $\left(\hat{n}_{n}, \hat{s}_{n}, \hat{z}\right)$ constitute a right-handed coordinate system. The width of the $n^{t h}$ segment is represented by $w_{n}$. In addition, the coordinates of the mid point of the $n^{\text {th }}$ segment is denoted by $\left(x_{n}^{s}, y_{n}^{s}\right)$. 


\subsection{1 $\quad \mathrm{Z}^{E}$ : Edge Impedance Matrix}

The Edge Impedance Matrix denoted by $\mathbf{Z}^{E}$ accounts for the interaction between edge ports in absence of the slot. Hence, it is the same as the impedance matrix defined in 2.72 .

\subsection{2 $\quad \mathrm{H}^{S E}$ : Edge-To-Slot Transfer Matrix}

$\mathbf{H}^{S E}$ accounts for the transverse magnetic field produced by edge currents on the slot and was defined in 3.102b. In order to find transverse magnetic field produced by edge currents, it is assumed that away from the slot, the structure is essentially two dimensional and only supports $T M^{z}$ modes. Equation 2.54 gives the electric field at an arbitrary point $\left(x_{0}, y_{0}\right)$ inside a closed contour $C$ in terms of the electric field and its normal derivative on the contour, which after discretization can be written as:

$$
E_{z}\left(x_{0}, y_{0}\right)=-\frac{\omega \mu}{4 w_{j}} \sum_{j=1}^{N^{E}} \bar{I}_{j}^{E} \int_{w_{j}} H_{0}^{(2)}(k \rho) d s-\frac{k}{4 j d} \sum_{j=1}^{N^{E}} \bar{V}_{j}^{E} \int_{w_{j}} \cos \theta H_{1}^{(2)}(k \rho) d s
$$

where $\rho$ and $\cos \theta$ have similar definitions as in Fig. 2.6, except for the fact that the point $\left(x_{n}^{s}, y_{n}^{s}\right)$ is replaced by the point $\left(x_{0}, y_{0}\right) . \bar{I}_{j}^{E}$ is the total current flowing inward at the $j^{\text {th }}$ port. By substituting 2.72 into 3.124 we will have:

$$
E_{z}\left(x_{0}, y_{0}\right)=\sum_{n=1}^{N^{E}} \bar{I}_{n}^{E}\left[-\frac{\omega \mu}{4 w_{n}} \int_{w_{n}} H_{0}^{(2)}(k \rho) d s-\frac{k}{4 j d} \sum_{j=1}^{N^{E}} \mathbf{Z}_{j n}^{E} \int_{w_{j}} \cos \theta H_{1}^{(2)}(k \rho) d s\right]
$$

Since it's been assumed that away from the slot the fields are essentially $T M^{z}$, transverse magnetic field components and consequently $\mathbf{H}^{S E}$ elements can be calculated from 2.1b, 2.1c, and 3.102b.

Testing functions are chosen to be the same as the basis functions. This choice gives not only a variational form, but also a physical meaning to matrix elements in terms of reaction between electric and magnetic current sources. In addition, as it will be shown in the next section, in this case $\mathbf{H}^{E S}$ becomes the transpose of $\mathbf{H}^{S E}$. 


\subsection{3 $\quad \mathbf{H}^{E S}$ : Slot-to-Edge Transfer Matrix}

It was stated that if the same set of functions is used for both expansion and testing, $\mathbf{H}^{E S}$ becomes the transpose of $\mathbf{H}^{S E}$. In this section, the proof based on Lorentz reciprocity theorem [41] will be given.

Since it is assumed that the circuit is enclosed by PMC walls on the sides and PEC walls from top and bottom, $\hat{n} \times \vec{H}=0$ on the sidewalls and $\hat{n} \times \vec{E}=0$ on

the patch and the ground plane. Therefore Lorentz reciprocity theorem takes the following form:

$$
\iint_{V} \int\left(\vec{E}^{1} \cdot \vec{J}^{2}-\vec{H}^{1} \cdot \vec{M}^{2}\right) d v=\iint_{V} \int\left(\vec{E}^{2} \cdot \vec{J}^{1}-\vec{H}^{2} \cdot \vec{M}^{1}\right) d v
$$

Suppose that all edge ports are open-circuited except for the $n^{\text {th }}$ port which is excited by $\bar{I}_{n}^{E}$ flowing into the circuit. We write the reciprocity equation for the reaction between $\bar{I}_{n}^{E}$ and the $m^{t h}$ basis function, $\vec{M}_{m}$. We have:

$$
\left\{\begin{array}{lll}
\vec{J}^{1}=\hat{z} \bar{I}_{n}^{E} / w_{n} & , & \vec{M}^{1}=0 \\
\vec{J}^{2}=0 & , & \vec{M}^{2}=\vec{M}_{m}
\end{array}\right.
$$

By substituting these into 3.126, we have:

$$
\begin{aligned}
-\iint_{\text {slot }} \vec{H}^{1}\left(\bar{I}_{n}^{E}\right) \cdot \vec{M}_{m} d S & =-\left\langle\vec{H}_{T}\left(\bar{I}_{n}^{E}\right), \vec{M}_{m}\right\rangle \\
& =d \int E^{2 z}\left(\vec{M}_{m}\right) \frac{\bar{I}_{n}^{E}}{w_{n}} d s=-\bar{V}_{n}^{E}\left(\vec{M}_{m}\right) \bar{I}_{n}^{E}
\end{aligned}
$$

By comparing 3.128 with $3.102 \mathrm{a}$ and $3.102 \mathrm{~b}$ it can be seen that:

$$
\mathbf{H}_{n m}^{E S}=\bar{V}_{n}^{E}\left(\vec{M}_{m}\right)=\frac{\left\langle\vec{H}_{T}\left(\bar{I}_{n}^{E}\right), \vec{M}_{m}\right\rangle}{\bar{I}_{n}^{E}}=\mathbf{H}_{m n}^{S E}
$$

which proves the fact that $\mathbf{H}^{E S}$ is the transpose of $\mathbf{H}^{S E}$. 


\subsection{4 $\quad \mathrm{Y}^{S}$ : Slot Admittance Matrix}

Slot admittance matrix accounts for the interaction among slot ports. In order to calculate this matrix we need the magnetic field produced by the equivalent magnetic sources over the slot at any point inside a planar cavity of arbitrary shape surrounded by PMC walls. Since the modal expansion inside such a cavity is unknown, we propose the following method.

We first calculate the fields due to $\vec{M}^{\text {slot }}$ assuming that it is radiating in a laterally infinite (unbounded) parallel-plate waveguide. These fields, hereinafter, will be referred to as incident fields. Incident fields can be found by either modal expansion or image series. Both these methods show very poor convergence behavior either close to or far from the source [57]. Therefore complex images method [57] has been used to find the incident fields. This is the subject of the next chapter. Here we assume that these fields are denoted by $\vec{H}^{p p}(x, y, z)$. The incident fields can be written as:

$$
\vec{H}^{p p}(x, y, z)=\sum_{n=1}^{N^{S}} \bar{V}_{n}^{S} \vec{H}_{n}^{p p}(x, y, z)
$$

where $\vec{H}_{\ell}^{p p}(x, y, z)$ represents the magnetic field radiated by $\vec{M}_{\ell}$ in an unbounded parallel-plate waveguide. If the periphery of the circuit is covered by PMC walls, incident fields will be reflected from the boundary. Equivalently, it can be assumed that incident fields have induced some unknown magnetic current on the periphery, $\vec{M}^{\text {wall }}$, which acts as a source for reflected fields in a way that the tangential components of incident and reflected magnetic fields will cancel on the boundary, hence satisfying open-circuit boundary condition. This will allow us to find the induced magnetic current on the walls. The analysis can be drastically simplified if it is assumed that away from the slot, the fields are essentially two dimensional. Consequently, $\vec{M}^{\text {wall }}$ will only have a component tangent to the contour $C$ at any point along the boundary and we will be able to use 2.78 to find $\vec{M}^{\text {wall }}$ in terms of the tangential component of magnetic field on the boundary. The sampling points are taken to be the mid-point of each segment. From 2.78 we have:

$$
\bar{M}^{\text {wall }}=\mathbf{S}\left[\hat{s}_{m} \cdot \vec{H}_{m}\right]
$$

where

$$
\mathbf{S}_{m n}=\frac{w_{n}}{d} \mathbf{Z}_{m n}^{E}
$$

is the transformation matrix. By sampling $\vec{H}^{p p}$ at the same points along the bound- 
ary and enforcing the boundary condition on PMC walls we have:

$$
\hat{s}_{m} \cdot \vec{H}_{m}=-\sum_{n=1}^{N^{S}} \bar{V}_{n}^{S}\left[\hat{s}_{m} \cdot \vec{H}_{n}^{p p}\left(s_{m}\right)\right]
$$

or in matrix notation:

$$
\left[\hat{s}_{m} \cdot \vec{H}_{m}\right]=-\mathbf{H}_{s}^{p p} \bar{V}^{S}
$$

where the matrix $\mathbf{H}_{s}^{p p}$ is defined as $\mathbf{H}_{s, m n}^{p p}=\hat{s}_{m} \cdot \vec{H}_{n}^{p p}\left(s_{m}\right)$. By combining 3.131 and 3.134 , the induced magnetic current, i.e. $\bar{M}^{\text {wall }}$ can be given as:

$$
\bar{M}^{\text {wall }}=-\mathbf{S} \cdot \mathbf{H}_{s}^{p p} \bar{V}^{S}
$$

Cavity fields satisfying all boundary conditions can now be obtained by superposition of the fields radiated by $\vec{M}^{\text {slot }}$ in an unbounded parallel-plate waveguide and $\bar{M}^{\text {wall }}$. Using complex images method, magnetic field radiated by a horizontal magnetic dipole (HMD) in an arbitrary direction specified by a unit vector $\hat{c}$, can be written as:

$$
\hat{c} \cdot \vec{H}^{p p}\left(x_{0}, y_{0}\right)=\sum_{n=1}^{N^{S}} \hat{c} \cdot \vec{H}_{n}^{p p}\left(x_{0}, y_{0}\right) \bar{V}_{n}^{S}
$$

The magnetic field due to $\bar{M}^{\text {wall }}$ can be calculated from 2.79. The superscript "bi" will be used hereinafter to denote fields by boundary integral method, which is another name for contour integral method. This is in order to avoid confusion as the superscript "ci" will be used in the next chapter to denote fields from complex images method.

$$
\hat{c} \cdot \vec{H}^{b i}\left(x_{0}, y_{0}\right)=\sum_{\ell=1}^{N^{S}} \bar{V}_{\ell}^{S} \hat{c} \cdot \vec{H}_{\ell}^{b i}\left(x_{0}, y_{0}\right)
$$

where

$$
\begin{aligned}
\vec{H}_{\ell}^{b i}\left(x_{0}, y_{0}\right)= & \frac{k^{2}}{4 \omega \mu} \sum_{j=1}^{N^{E}}\left[\mathbf{S} \cdot \mathbf{H}_{s}^{p p}\right]_{j \ell}\left[\frac{\hat{\rho}_{j+1} H_{1}^{(2)}\left(k \rho_{j+1}\right)-\hat{\rho}_{j} H_{1}^{(2)}\left(k \rho_{j}\right)}{k}-\hat{s}_{j} \int_{w_{j}} H_{0}^{(2)}(k \rho) d s\right]+ \\
& -\frac{k}{4 j} \sum_{j=1}^{N^{E}} \mathbf{H}_{s, j \ell}^{p p} \int_{w_{j}} \hat{\varphi} H_{1}^{(2)}(k \rho) d s
\end{aligned}
$$


and the total magnetic field will be:

$$
\begin{aligned}
\hat{c} \cdot \vec{H}^{t o t}\left(x_{0}, y_{0}\right) & =\hat{c} \cdot \vec{H}^{p p}\left(x_{0}, y_{0}\right)+\hat{c} \cdot \vec{H}^{b i}\left(x_{0}, y_{0}\right) \\
& =\sum_{n=1}^{N^{S}} \overline{V_{n}^{S}}\left[\hat{c} \cdot \vec{H}_{n}^{p p}\left(x_{0}, y_{0}\right)+\hat{c} \cdot \vec{H}_{n}^{b i}\left(x_{0}, y_{0}\right)\right]
\end{aligned}
$$

As can be seen from 3.139, the total magnetic field in an irregularly shaped cavity surrounded by PMC walls and excited by the equivalent magnetic current over the slot can be calculated at any point inside the cavity, in particular over the slot. If $\hat{c}$ is taken to be the direction vector for $m^{\text {th }}$ basis function, slot admittance matrix can be calculated as follows:

$$
\mathbf{Y}_{m n}^{S}=\mathbf{Y}_{m n}^{p p}+\mathbf{Y}_{m n}^{b i}
$$

where

$$
\begin{aligned}
\mathbf{Y}_{m n}^{p p} & =\left\langle\vec{H}_{n}^{p p}, \vec{M}_{m}\right\rangle \\
\mathbf{Y}_{m n}^{b i} & =\left\langle\vec{H}_{n}^{b i}, \vec{M}_{m}\right\rangle
\end{aligned}
$$

Compared to other matrices, $\mathbf{Y}^{p p}$ is the most computationally-intensive. However, it has the interesting property that $\mathbf{Y}_{m n}^{p p}$ depends only on $|m-n|$. Hence, $\mathbf{Y}^{p p}$ can be constructed from the elements of any row or column. Remembering that $\mathbf{Y}^{p p}$ is the slot admittance matrix radiating into a homogenous parallel-plate waveguide, this is intuitively the case. Nonetheless, a proof is given in Appendix $\mathrm{C}$ for a special case of a narrow slot and rooftop basis functions with uniformly spaced centre points. 


\section{Chapter 4}

\section{Complex Images Method}

\subsection{Introduction}

A rigorous analysis of conducting objects embedded in a planar stratified medium consisting of an arbitrary number of dielectric layers possibly backed by ground planes on one or both sides can be most conveniently formulated using Mixed Potential Integral Equation (MPIE), in which conducting objects are replaced by equivalent charges and currents on their surfaces and fields are expressed in terms of scalar and vector potentials [58], [59]. Analytic expressions for spectral domain potentials can be derived in a variety of ways. Spatial expressions can be obtained by evaluating the inverse Hankel transforms of spectral expressions [51], [60]. An important advantage of MPIE for layered media is that the kernel of potential functions are weakly singular compared to kernel functions associated with fields which are obtained by differentiation of potentials [59], [60]. This advantage of MPIE for layered media was first observed by Mosig and Gardiol [60], [61], [62], [63]. Nonetheless, computation of inverse Hankel transforms, also known as Sommerfeldtype integrals, is a difficult and time-consuming task. This is mainly due to the oscillatory and singular behavior of integrands [60]. These singularities consist of isolated poles and branch cuts which occur in complex conjugate pairs in the second and fourth quadrants of the complex $k_{\rho}$ plane for a $e^{j \omega t}$ time variation [64], where $k_{\rho}$ is the transverse propagation constant. Isolated poles correspond to surface waves and branch cuts represent radiation. For lossless media, isolated poles appear on the real axis [60], which necessitates indentation of the integration path into the first and third quadrants [65], [66]. Branch cuts only occur for vertically unbounded layers, i.e. the top or bottom layers when they are not backed by ground planes. 


\section{CHAPTER 4. COMPLEX IMAGES METHOD}

Analytical evaluation of transform integrals is only possible for a very limited group of functions encountered in wave propagation. Hence numerical integration, which is quite computationally intensive for Sommerfeld-type integrals, is unavoidable. In order to avoid these time-consuming numerical integrations a method called Complex Image Method or Discrete Complex Image Method (DCIM) was developed for modeling of microwave integrated circuits (MIC's) and microstrip antennas [67], [68].

Complex images refer to finite number of images in complex locations that can replace an infinite number of real images. In an effort to derive simple expressions for spatial Green's functions of microstrip structures, real image series solution was developed in static case from a model of charge images by Silvester [69], and was later extended to dynamic case by Chow for thin microstrip substrates [70], [71]. However, for thick substrates and large source-to-field distances, this dynamic model deteriorates rapidly. This can be attributed to the fact that the dynamic model essentially neglects surface and leaky wave modes [68]. An exact image method was later developed by Lindell and Alanen [72], [73], [74], [75], [76], which was based on using Mittag-Leffler expansion of generalized reflection coefficients of the layered media to analytically calculate their inverse Laplace transforms. In fact, the exact image method avoided Sommerfeld integrals by replacing them with alternate infinite integrals, which later proved to be still time-consuming [67]. Finally Chow introduced complex images for calculating spatial Green's functions of vector and scalar potentials with an HED located over a thick substrate, which avoided numerical integration of any kind [68]. Other authors later extended complex images to a more general class of microstrip geometries with both a substrate and a superstrate [77], [78], and even for non-symmetrical components of the Green's function [79].

Another method closely associated with complex images is the Generalized Pencil of Function (GPOF) which has been used to find closed-form Green's functions for a general multi-layer structure with a source of any type and in any direction embedded in any of the layers [80], [81], [82], [83], [84], [85]. GPOF is more robust than complex images for large number of layers and complex problems. But in the case of this thesis, the added complexity in using GPOF instead of complex images is not justified and hence complex images has been used throughout this thesis.

Nonetheless, complex images method has its own shortcomings as it has no built-in convergence measures and its accuracy cannot be trusted a priori without checking its results against those obtained from more established methods [59]. There is no reliable automated procedure for extraction of surface-wave poles. There is also problems when the source is embedded in bounded regions, for which a 
CHAPTER 4. COMPLEX IMAGES METHOD

Table 4.1: Potential functions for different kinds of sources

\begin{tabular}{lllll}
\hline$V E D$ & $G_{A}^{z z}$ & $G_{q, e}^{z}$ & - & - \\
$V M D$ & - & - & $G_{F}^{z z}$ & $G_{q, m}^{z}$ \\
$H E D$ & $G_{A}^{x x}, G_{A}^{z x}$ & $G_{q, e}^{x}$ & - & - \\
$H M D$ & - & - & $G_{F}^{x x}, G_{F}^{z x}$ & $G_{q, m}^{x}$ \\
\hline
\end{tabular}

solution has been proposed [86], [87]. Others include lack of clear instructions on the number of extracted quasi-static terms [86] and if extraction of surface-wave poles is necessary at close distances from the source [88].

In spite of these shortcomings, complex images method remains to be a very fast and reliable method, when its accuracy can be established. We have used complex images for calculating transverse magnetic fields due to an HMD in a parallel plate waveguide. We have also developed programs for both HED and HMD in a threelayer structure shown in Fig. 4.2. These will be used in the future work that will be discussed in the closing chapter.

In the next section, analytic expressions for vector and scalar potentials in spectral domain will be derived. Formulation of complex images for the problem in hand will follow in a separate section. The procedure outlined here closely follows that of [64]. However, we have rearranged and in some cases generalized the expressions given in [64] for a more systematic presentation that is more suitable for this thesis.

\subsection{Spectral Green's Functions for a Horizontal Electric and Magnetic Dipoles}

Analytic expressions of Spectral Green's functions for stratified media can be derived in a variety of ways. For a general stratified media with either electric or magnetic dipoles, there is a total of 10 distinct potential functions [89]. These are listed in Table 4.1. $V, H, E$, and $M$ signify vertical, horizontal, electric, and magnetic attributes, respectively. For example, $H M D$ stands for Horizontal Magnetic Dipole. 


\section{CHAPTER 4. COMPLEX IMAGES METHOD}

Chow et al. [89] build an analogy between TEM transmission lines and plane wave propagation in planarly layered media and derive the spectral Green's functions by cascading these transmission lines This approach is very systematic and can be conveniently applied to any number of layers. A similar but less systematic approach has also been used by Michalski and Mosig [59]. Another systematic approach amenable to programming is based on enforcing the boundary conditions on potentials in spectral domain [90]. The spectral potentials can also be obtained in terms of Fresnel reflection coefficients from longitudinal field components $\left(E_{z}\right.$ and $H_{z}$, where $z$ is the direction of stratification). This approach gives better physical insight into the problem and has been utilized in this thesis. The expressions given for vector and scalar potentials are new in presentation, though it cannot be denied that they are in fact rearrangements and in some cases generalizations of the expressions appeared in the literature.

\subsubsection{Reflection and Transmission of Plane-Waves in Planarly Layered Media}

It can be shown that for an $N$-layer medium, such as the one shown in Fig. 4.1, the generalized reflection coefficient at the interface between regions $i$ and $i+1$, denoted by $\tilde{R}_{i, i+1}\left(\tilde{R}_{i, i+1}^{T E}\right.$ for $T E$ waves and $\tilde{R}_{i, i+1}^{T M}$ for $T M$ waves), can be given by the following recursive relation in terms of $\tilde{R}_{i+1, i+2}[64]$ :

$$
\tilde{R}_{i, i+1}=\frac{R_{i, i+1}+\tilde{R}_{i+1, i+2} e^{-2 j k_{i+1, z}\left(d_{i+1}-d_{i}\right)}}{1+R_{i, i+1} \tilde{R}_{i+1, i+2} e^{-2 j k_{i+1, z}\left(d_{i+1}-d_{i}\right)}}
$$

where $R_{i, i+1}$ is the Fresnel reflection coefficient for the downgoing wave in region $i$ reflected by region $i+1$ and we have:

$$
\begin{aligned}
R_{i, i+1}^{T E} & =\frac{\mu_{i+1} k_{i z}-\mu_{i} k_{i+1, z}}{\mu_{i+1} k_{i z}+\mu_{i} k_{i+1, z}} \\
R_{i, i+1}^{T M} & =\frac{\varepsilon_{i+1} k_{i z}-\varepsilon_{i} k_{i+1, z}}{\varepsilon_{i+1} k_{i z}+\varepsilon_{i} k_{i+1, z}}
\end{aligned}
$$

Note that $R_{i, i+1}^{T E}$ and $R_{i, i+1}^{T M}$ are reflection coefficients for the transverse electric and magnetic fields, respectively. $k_{i z}$ is the propagation constant in $z$-direction for the $i^{\text {th }}$ layer. Since $\tilde{R}_{N, N+1}=0$, equation 4.142 can be solved recursively in all regions. $\tilde{R}_{i, i+1}$ can represent the generalized reflection coefficient for either $T E$ or $T M$ waves 


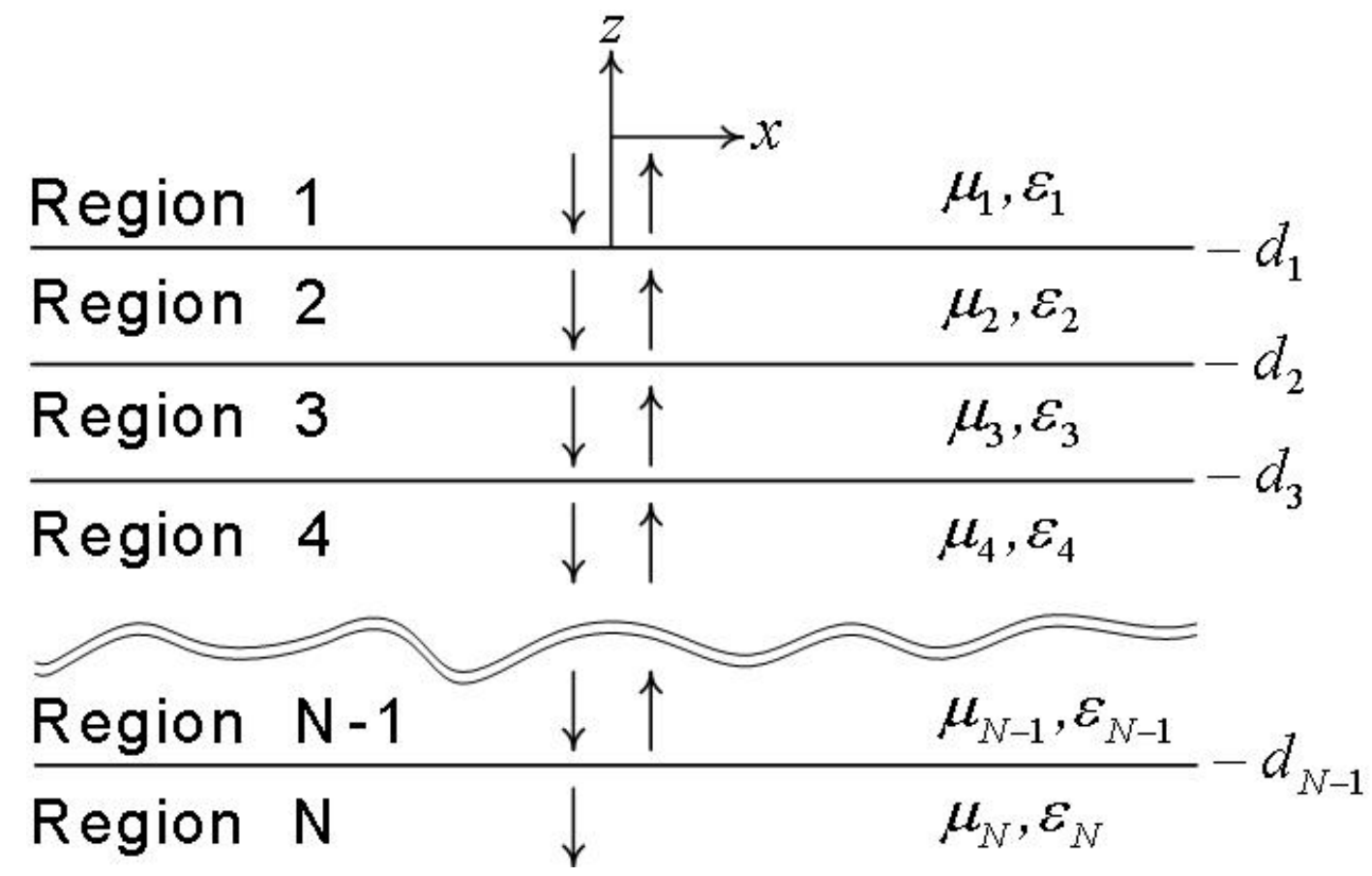

Figure 4.1: Reflection and transmission in a multilayered media

if all other reflection coefficients in the recursive relation correspond to $T E$ or $T M$ waves, respectively. Each of transverse field components $\left(E_{x}\right.$ and $E_{y}$ for $T E$ waves, $H_{x}$ and $H_{y}$ for $T M$ waves) in the $i^{\text {th }}$ region assumes the form [64]:

$$
E_{x}, E_{y}, H_{x}, H_{y} \propto A_{i}\left[e^{j k_{i z} z}+\tilde{R}_{i, i+1} e^{-j k_{i z} z-2 j k_{i z} d_{i}}\right]
$$

$A_{i}$ 's are given by the following recursive relation:

$$
A_{i} e^{-j k_{i z} d_{i-1}}=\frac{T_{i-1, i} A_{i-1} e^{-j k_{i-1, z} d_{i-1}}}{1-R_{i, i-1} \tilde{R}_{i, i+1} e^{-2 j k_{i z}\left(d_{i}-d_{i-1}\right)}}
$$

where $T_{i-1, i}=1+R_{i-1, i}$ is the Fresnel transmission coefficient for the downgoing wave in region $i-1$ to the region $i$. A generalized transmission coefficient can also be defined from region $m$ to region $n$ as:

$$
A_{n} e^{-j k_{n z} d_{n-1}}=\tilde{T}_{m n} A_{m} e^{-j k_{m z} d_{m}}
$$

It can be readily shown that:

$$
\tilde{T}_{m n}=S_{m, m+1} \prod_{\ell=m+1}^{n-1} e^{-j k_{\ell z}\left(d_{\ell}-d_{\ell-1}\right)} S_{\ell, \ell+1} \quad n>m
$$




\section{CHAPTER 4. COMPLEX IMAGES METHOD}

where

$$
S_{\ell, \ell+1}=\frac{T_{\ell, \ell+1}}{1-R_{\ell+1, \ell} \tilde{R}_{\ell+1, \ell+2} e^{-2 j k_{\ell+1, z}\left(d_{\ell+1}-d_{\ell}\right)}}
$$

This is for the case when the source is on top of a layered medium. If the source is embedded in the layered medium, say in region $m$, then the $z$ variation of the solution within the same layer for a symmetric source can be written as [64]:

$$
F_{+}\left(z, z^{\prime}\right)=\frac{\left[e^{j k_{m z} z^{\prime}}+e^{-j k_{m z}\left(z^{\prime}+2 d_{m}\right)} \tilde{R}_{m, m+1}\right]\left[e^{-j k_{m z} z}+e^{j k_{m z}\left(z+2 d_{m-1}\right)} \tilde{R}_{m, m-1}\right]}{\tilde{M}_{m}}
$$

for $z \geqslant z^{\prime}$ (the subscript + indicates that $z \geqslant z^{\prime}$ ). We also have:

$$
\tilde{M}_{m}=1-\tilde{R}_{m, m+1} \tilde{R}_{m, m-1} e^{-2 j k_{m z}\left(d_{m}-d_{m-1}\right)}
$$

$z$ and $z^{\prime}$ are field and source points, respectively. By exchanging the locations of $z$ and $z^{\prime}$, equation 4.149 can also be used for $z^{\prime} \geqslant z$, i.e. $F_{-}\left(z, z^{\prime}\right)=F_{+}\left(z^{\prime}, z\right)$. This is for a symmetric source. A source is symmetric if its fields are symmetric about $z^{\prime}$ in a homogenous medium, i.e. $F\left(z, z^{\prime}\right)=e^{-j k_{m z}\left|z-z^{\prime}\right|}$ in homogenous medium. Similarly, a source is odd-symmetric if $F\left(z, z^{\prime}\right)=\partial e^{-j k_{m z}\left|z-z^{\prime}\right|} / \partial z^{\prime}$ in homogenous medium [64]. If the source is in region $m$ and the field in region $n$, where $n<m$, $F_{+}\left(z, z^{\prime}\right)$ can be shown to be given as [64]:

$$
\begin{aligned}
F_{+}\left(z, z^{\prime}\right)= & {\left[e^{-j k_{n z} z}+e^{j k_{n z}\left(z+2 d_{n-1}\right)} \tilde{R}_{n, n-1}\right]\left[e^{j k_{m z} z^{\prime}}+e^{-j k_{m z}\left(z^{\prime}+2 d_{m}\right)} \tilde{R}_{m, m+1}\right] \times } \\
& \frac{e^{-j k_{n z} d_{n}} \tilde{T}_{m n} e^{j k_{m z} d_{m-1}}}{\tilde{M}_{m} \tilde{M}_{n}}
\end{aligned}
$$

where the plus sign in subscript indicates that $z>z^{\prime}$ or $n<m$ as can be seen from Fig. 4.1. For $n>m$ or $z<z^{\prime}$ we have [64]:

$$
\frac{\mu_{n}}{k_{n z}} F_{-}\left(z^{\prime}, z\right)=\frac{\mu_{m}}{k_{m z}} F_{+}\left(z, z^{\prime}\right)
$$

By differentiating $F_{+}\left(z, z^{\prime}\right)$ and/or $F_{-}\left(z, z^{\prime}\right)$, the solutions corresponding to oddsymmetric sources can be obtained.

\subsubsection{Spatial Vector and Scalar Potentials for Multi-layered Media}

Spatial potentials can be expressed as inverse Hankel transforms of their spectral counterparts. It can be easily shown that plane waves reflecting from planarly 


\section{CHAPTER 4. COMPLEX IMAGES METHOD}

layered media can be decomposed into $T E$ and $T M$ waves [66]. On the other hand, by using the following identity, due to Sommerfeld, the fields due to an electric or magnetic dipole (point source) can be expanded into plane waves:

$$
\frac{e^{-j k r}}{r}=\int_{-\infty}^{+\infty} \frac{e^{-j k_{z}\left|z-z^{\prime}\right|}}{j 2 k_{z}} H_{0}^{(2)}\left(k_{\rho} \rho\right) k_{\rho} d k_{\rho}
$$

where $r=\sqrt{\left(z-z^{\prime}\right)^{2}+\rho^{2}}, k_{\rho}=\sqrt{k^{2}-k_{z}^{2}}$. Hence, the procedure for calculating the fields due to a point source on top of or embedded in a planarly layered medium, can be summarized as follows:

1. Characterizing $T E$ and $T M$ waves for the source in a homogenous medium

2. Expanding the fields into plane waves

3. Studying the propagation of these $T E$ and $T M$ plane waves in the layered medium

The fields due to an electric dipole in an arbitrary direction designated by the unit vector $\hat{\alpha}$, i.e. $\vec{J}=\hat{\alpha} I \ell \delta(\vec{r})$, and located in a homogenous medium, can be given as:

$$
\begin{aligned}
& \vec{E}(\vec{r})=-j \omega \mu_{s}\left(\overline{\mathbf{I}}+\frac{\nabla \nabla}{k_{s}^{2}}\right) \cdot \hat{\alpha} I \ell \frac{e^{-j k_{s} r}}{4 \pi r} \\
& \vec{H}(\vec{r})=\nabla \times \hat{\alpha} I \ell \frac{e^{-j k_{s} r}}{4 \pi r}
\end{aligned}
$$

where $I \ell$ is the current moment and $\overline{\mathbf{I}}$ is the unit dyadic. The subscript $s$ denotes source region.

For an HED pointing in $\hat{\alpha}=\hat{x}$ direction and radiating in a homogenous medium we have:

$$
\begin{aligned}
& E_{z}=\frac{j I \ell}{4 \pi \omega \varepsilon_{s}} \frac{\partial}{\partial x} \int_{-\infty}^{+\infty} \frac{\partial e^{-j k_{s z}\left|z-z^{\prime}\right|} / \partial z^{\prime}}{j 2 k_{s z}} H_{0}^{(2)}\left(k_{\rho} \rho\right) k_{\rho} d k_{\rho} \\
& H_{z}=-\frac{I \ell}{4 \pi} \frac{\partial}{\partial y} \int_{-\infty}^{+\infty} \frac{e^{-j k_{s z}\left|z-z^{\prime}\right|}}{j 2 k_{s z}} H_{0}^{(2)}\left(k_{\rho} \rho\right) k_{\rho} d k_{\rho}
\end{aligned}
$$




\section{CHAPTER 4. COMPLEX IMAGES METHOD}

As can be seen from 4.155a and 4.155b, an HED is an odd-symmetric source for $T M$ waves (downgoing waves have negative sign) but a symmetric source for $T E$ waves. When the medium is stratified in the $z$ direction, $\partial e^{-j k_{s z}\left|z-z^{\prime}\right|} / \partial z^{\prime}$ and $e^{-j k_{s}\left|z-z^{\prime}\right|}$ in $4.155 \mathrm{a}$ and $4.155 \mathrm{~b}$ have to be replaced by $\partial F^{T M}\left(z, z^{\prime}\right) / \partial z^{\prime}$ and $F^{T E}\left(z, z^{\prime}\right)$ from the previous section, respectively. The superscripts $T M$ and $T E$ indicate that the reflection and transmission coefficients in $F\left(z, z^{\prime}\right)$ correspond to $T M$ or $T E$ waves. Therefore we will have:

$$
\begin{aligned}
& E_{z}=\frac{j I \ell}{4 \pi \omega \varepsilon_{s}} \frac{\partial}{\partial x} \int_{-\infty}^{+\infty} \frac{\partial F^{T M}\left(z, z^{\prime}\right) / \partial z^{\prime}}{j 2 k_{s z}} H_{0}^{(2)}\left(k_{\rho} \rho\right) k_{\rho} d k_{\rho} \\
& H_{z}=-\frac{I \ell}{4 \pi} \frac{\partial}{\partial y} \int_{-\infty}^{+\infty} \frac{F^{T E}\left(z, z^{\prime}\right)}{j 2 k_{s z}} H_{0}^{(2)}\left(k_{\rho} \rho\right) k_{\rho} d k_{\rho}
\end{aligned}
$$

The fields due to an HMD pointing in $\hat{\alpha}=\hat{x}$ direction can be obtained by using concept of duality. A magnetic dipole is simulated by an infinitesimal electric current loop antenna. It can be shown that by making the replacement $I \ell \rightarrow$ $-j \omega \mu_{s} I A_{\ell}$, where $A_{\ell}$ is the area of the loop carrying the current $I$, and subsequent application of duality [41], the fields due to an HMD can be obtained from HED case [91]:

$$
\begin{aligned}
& H_{z}=\frac{I A_{\ell}}{4 \pi} \frac{\partial}{\partial x} \int_{-\infty}^{+\infty} \frac{\partial F^{T E}\left(z, z^{\prime}\right) / \partial z^{\prime}}{j 2 k_{s z}} H_{0}^{(2)}\left(k_{\rho} \rho\right) k_{\rho} d k_{\rho} \\
& E_{z}=-\frac{j \omega \mu_{s} I A_{\ell}}{4 \pi} \frac{\partial}{\partial y} \int_{-\infty}^{+\infty} \frac{F^{T M}\left(z, z^{\prime}\right)}{j 2 k_{s z}} H_{0}^{(2)}\left(k_{\rho} \rho\right) k_{\rho} d k_{\rho}
\end{aligned}
$$

Vector and scalar potentials can now be obtained from $E_{z}$ and $H_{z}$. For an HED in $x$ direction with unit current moment, i.e. $I \ell=1$, we have:

$$
\begin{gathered}
G_{A}^{x x}=\frac{\mu_{f}}{4 \pi} \int_{-\infty}^{+\infty} \frac{F^{T E}\left(z, z^{\prime}\right)}{j 2 k_{s z}} H_{0}^{(2)}\left(k_{\rho} \rho\right) k_{\rho} d k_{\rho} \\
G_{A}^{z x}=\frac{\mu_{f}}{4 \pi} \frac{\partial}{\partial x} \int_{-\infty}^{+\infty} \frac{1}{k_{\rho}^{2}}\left[\frac{\varepsilon_{f}}{\varepsilon_{s}} \frac{\partial F^{T M}\left(z, z^{\prime}\right)}{\partial z}-\frac{\partial F^{T E}\left(z, z^{\prime}\right)}{\partial z}\right] \frac{1}{j 2 k_{s z}} H_{0}^{(2)}\left(k_{\rho} \rho\right) k_{\rho} d k_{\rho}
\end{gathered}
$$




\section{CHAPTER 4. COMPLEX IMAGES METHOD}

The scalar potential is defined as [89]:

$$
\frac{\partial G_{q, e}^{x}}{\partial x}=\frac{1}{\mu_{f} \varepsilon_{f}}\left(\frac{\partial G_{A}^{x x}}{\partial x}+\frac{\partial G_{A}^{z x}}{\partial z}\right)
$$

From which we will have:

$$
G_{q, e}^{x}=\frac{1}{4 \pi \varepsilon_{f}} \int_{-\infty}^{+\infty}\left\{\left(1+\frac{k_{f z}^{2}}{k_{\rho}^{2}}\right) F^{T E}\left(z, z^{\prime}\right)-\frac{k_{f z}^{2}}{k_{\rho}^{2}} \frac{\varepsilon_{f}}{\varepsilon_{s}} F^{T M}\left(z, z^{\prime}\right)\right\} \frac{1}{j 2 k_{s z}} H_{0}^{(2)}\left(k_{\rho} \rho\right) k_{\rho} d k_{\rho}
$$

where the subscript $f$ indicates field region. Similarly, for an HMD in $x$ direction with $-j \omega \mu_{s} I A_{\ell}=1$, it can be shown that:

$$
\begin{gathered}
G_{F}^{x x}=\frac{1}{4 \pi} \int_{-\infty}^{+\infty} \frac{F^{T M}\left(z, z^{\prime}\right)}{j 2 k_{s z}} H_{0}^{(2)}\left(k_{\rho} \rho\right) k_{\rho} d k_{\rho} \\
G_{F}^{z x}=\frac{1}{4 \pi} \frac{\partial}{\partial x} \int_{-\infty}^{+\infty} \frac{1}{k_{\rho}^{2}}\left[\frac{\mu_{f}}{\mu_{s}} \frac{\partial F^{T E}\left(z, z^{\prime}\right)}{\partial z}-\frac{\partial F^{T M}\left(z, z^{\prime}\right)}{\partial z}\right] \frac{1}{j 2 k_{s z}} H_{0}^{(2)}\left(k_{\rho} \rho\right) k_{\rho} d k_{\rho}
\end{gathered}
$$

Except for a scaling factor, the scalar potential for an HMD is defined in the same way as for an HED [89]:

$$
\frac{\partial G_{q, m}^{x}}{\partial x}=\frac{1}{\mu_{f}}\left(\frac{\partial G_{F}^{x x}}{\partial x}+\frac{\partial G_{F}^{z x}}{\partial z}\right)
$$

which yields:

$$
G_{q, m}^{x}=\frac{1}{4 \pi \mu_{f}} \int_{-\infty}^{+\infty}\left[\left(1+\frac{k_{f z}^{2}}{k_{\rho}^{2}}\right) F^{T M}\left(z, z^{\prime}\right)-\frac{k_{f z}^{2}}{k_{\rho}^{2}} \frac{\mu_{f}}{\mu_{s}} F^{T E}\left(z, z^{\prime}\right)\right] \frac{1}{j 2 k_{s z}} H_{0}^{(2)}\left(k_{\rho} \rho\right) k_{\rho} d k_{\rho}
$$

In this thesis we are mainly interested in a structure with three dielectric layers backed by a ground plane, as shown in Fig. 4.2. The results for such a structure with an HED embedded in region 2 are already given in [78]. For an HMD, similar expressions can be derived as follows:

$$
\begin{aligned}
G_{F}^{x x} & =\frac{1}{4 \pi} \int_{-\infty}^{+\infty} \tilde{G}_{F}^{x x} H_{0}^{(2)}\left(k_{\rho} \rho\right) k_{\rho} d k_{\rho} \\
G_{q, m}^{x} & =\frac{1}{4 \pi} \int_{-\infty}^{+\infty} \tilde{G}_{q, m}^{x} H_{0}^{(2)}\left(k_{\rho} \rho\right) k_{\rho} d k_{\rho}
\end{aligned}
$$




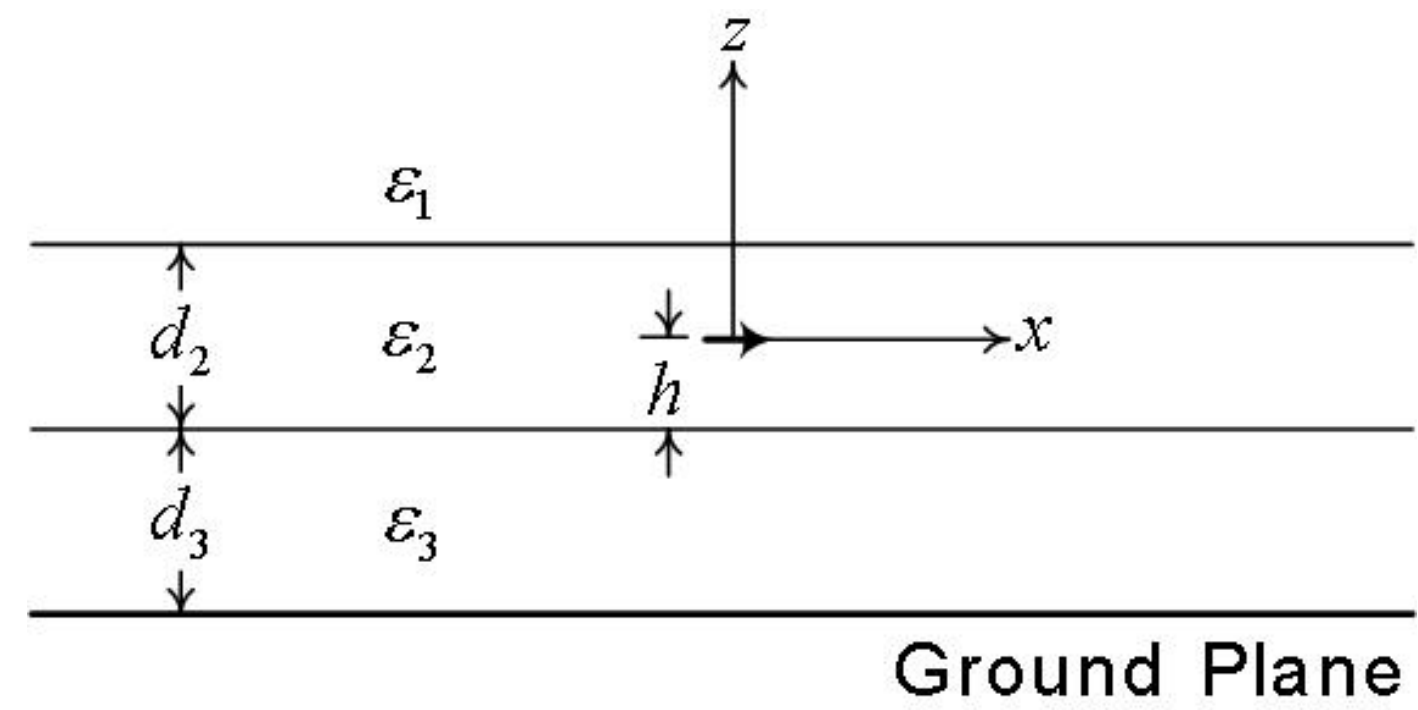

Figure 4.2: Three-layer structure backed by a ground plane

where

$$
\begin{aligned}
\tilde{G}_{F}^{x x}= & \frac{e^{-j k_{2 z}}+\bar{R}_{T M}^{+} e^{-j k_{2 z}\left(2 d_{2}-2 h-z\right)}-\bar{R}_{T M}^{-} e^{-j k_{2 z}(z+2 h)}}{j 2 k_{2 z}} \\
\tilde{G}_{q, m}^{x}= & \frac{1}{\mu_{2}} \frac{e^{-j k_{2 z}}+\left(\bar{R}_{T M}^{+}+\bar{R}_{q}^{+}\right) e^{-j k_{2 z}\left(2 d_{2}-2 h-z\right)}-\left(\bar{R}_{T M}^{-}+\bar{R}_{q}^{-}\right) e^{-j k_{2 z}(z+2 h)}}{j 2 k_{2 z}} \\
& \left.\bar{R}_{T E, T M}^{+}=\frac{1 \pm \tilde{R}_{21}^{T E, T M} e^{-j 2 k_{2 z}(z+h)}}{1-\tilde{R}_{21}^{T E, T M} \tilde{R}_{23}^{T E, T M} e^{-j 2 k_{2 z} d_{2}}} \tilde{R}_{23}^{T E, T M}\right\}+ \text { for } T M \\
& \left.\bar{R}_{T E, T M}^{-}=-\frac{1 \pm \tilde{R}_{23}^{T E, T M} e^{-j 2 k_{2 z}\left(d_{2}-z-h\right)}}{1-\tilde{R}_{21}^{T E, T M} \tilde{R}_{23}^{T E, T M} e^{-j 2 k_{2 z} d_{2}}} \tilde{R}_{21}^{T E, T M}\right\}+ \text { for } T E \\
& \left.\bar{R}_{q}^{ \pm}=\frac{k_{2 z}^{2}}{k_{\rho}^{2}}\left(\bar{R}_{T E}^{ \pm}+\bar{R}_{T M}^{ \pm}\right)\right\}
\end{aligned}
$$

It seems that $\bar{R}_{q}^{ \pm}$has a second-order singularity at $k_{\rho}=0$. As a matter of fact this is not the case. It can be shown that this singularity is of removable type (the numerator has a second-order zero at $k_{\rho}=0$ ). We preferred to remove this singularity analytically. The procedure is straightforward but very tedious. However, removal of the singularity is essential to the convergence of the complex images method. 


\section{CHAPTER 4. COMPLEX IMAGES METHOD}

\subsection{Complex Images Method}

The basic idea of complex images is extraction of quasi-static and surface-wave terms and approximating the remaining part with a few images with complex amplitudes at complex locations. All these three parts contribute to the solution, but each dominates in a certain region. Surface-wave and quasi-static terms dominate in the far-field region of dielectric interfaces and near-field region, respectively. Complex images correspond to leaky waves and are very important in intermediate region [68]. Here we will only give a brief introduction to complex images.

Consider the structure shown in Fig. 4.2. In implementing complex images method, spectral Green's functions are first broken down into three parts corresponding to quasi-static terms, surface-wave poles, and the remaining part to be approximated by the sum of a few complex exponentials. Let $G$ and $\tilde{G}$ represent spatial and spectral Green's functions, respectively. They are related through the Hankel transform as follows:

$$
G=\frac{1}{4 \pi} \int_{-\infty}^{+\infty} \tilde{G} H_{0}^{(2)}\left(k_{\rho} \rho\right) k_{\rho} d k_{\rho}
$$

where $\tilde{G}$ can be written in terms of generalized reflection and transmission coefficients.

If the frequency is equal to zero, then $k_{m}$ 's are all zero and all $k_{m z}$ 's will be equal (static case). Since $k_{m z}^{2}=k_{m}^{2}-k_{\rho}^{2}$, if $k_{\rho} \longrightarrow \infty$, regardless of the frequency of operation, all $k_{m z}$ 's will be equal in the limit (quasi-static case). Let

$$
\lim _{k_{\rho} \longrightarrow \infty} \tilde{G}=\tilde{G}_{0}
$$

Therefore extracting quasi-static part will result in faster decay of remaining part of $\tilde{G}$ for large values of $k_{\rho}$. $\tilde{G}_{0}$ can be expanded in exponentials of the form $e^{-j k_{s z}\left( \pm z+z_{n}^{r i}\right)}$, where $z_{n}^{r i}$ is the location of $n^{t h}$ real image and depends on $d_{1}, d_{2}$, and $h$. Note that $z_{0}^{r i}=0$ which corresponds to the direct term (source in homogenous medium). By using Sommerfeld identity in 4.153, inverse Hankel transform of $\tilde{G}_{0}$, i.e. $G_{0}$, can be calculated analytically as sum of terms of the following form:

$$
\frac{\exp \left[-j k_{s} \sqrt{\rho^{2}+\left( \pm z+z_{n}^{r i}\right)^{2}}\right]}{4 \pi \sqrt{\rho^{2}+\left( \pm z+z_{n}^{r i}\right)^{2}}}
$$




\section{CHAPTER 4. COMPLEX IMAGES METHOD}

This is the same as the quasi-dynamic image model intuitively developed by Chow [71]. In most cases there is infinite number of these real images in quasi-static part of the solution. Apparently considering too many real images in quasi-static part of the solution contradicts the purpose of using complex images, hence the series of real images has to be truncated. There are no specific guidelines on selection of these real images, though it is obvious that direct term should always be included. Therefore let $G_{0}$ and $\tilde{G}_{0}$ denote truncated quasi-static solutions including the direct term and a few of its images with strong contributions. The remaining part of $\tilde{G}$, i.e. $\tilde{G}-\tilde{G}_{0}$, will now have a much faster decay for large values of $k_{\rho}$.

It is well known that a grounded dielectric slab supports guided modes that are loosely confined to the dielectric slab and decay away from the interface [41]. The statement can be made about any grounded multi-layer dielectric structure. These guided modes which are dominant in the far-field along the dielectric interfaces are referred to as surface-wave modes. It can be shown that these surface-wave modes correspond to the poles of $\tilde{G}$ on the real axis. These real poles can adversely affect the solution for small values of $k_{\rho}$ (far-field region along dielectric interfaces) even if the integration path is deformed so that it is not close to these poles anymore [78]. These poles can be found and extracted from $\tilde{G}-\tilde{G}_{0}$. Mathematically they can be represented as:

$$
\frac{2 k_{\rho p}\left(\text { Residue at } k_{\rho p}\right)}{k_{\rho}^{2}-k_{\rho p}^{2}}
$$

where $k_{\rho p}$ is the surface-wave pole. The contribution of these surface-wave poles to the spatial Green's function can be analytically evaluated via residue calculus as:

$$
\frac{1}{4 \pi}(-j 2 \pi) k_{\rho p} H_{0}^{(2)}\left(k_{\rho p} \rho\right){\underset{k_{\rho}}{\lim _{\rho}}}_{k_{\rho p}}\left(k_{\rho}-k_{\rho p}\right) \tilde{G}
$$

Let $\tilde{G}_{s w}$ and $G_{s w}$ represent the contribution of all surface-wave poles in spectral and spatial domains, respectively. Suppose that $\tilde{G}_{c i}=\tilde{G}-\tilde{G}_{0}-\tilde{G}_{s w}$. If the inverse Hankel transform of $\tilde{G}_{c i}$ can be calculated analytically, we will have a close-form solution for $G$ as the sum of three terms, i.e. $G=G_{c i}+G_{0}+G_{s w}$. To this end, $\tilde{G}_{c i}$ has to be approximated by a few complex exponentials as:

$$
\tilde{G}_{c i}=\sum_{i=1}^{N_{c i}} a_{i} e^{-b_{i} k_{s z}}
$$

Since $\tilde{G}_{c i}$ is a function which decays rapidly for large values of $k_{\rho}$ and is not singular on the real axis, if the infinite integral in inverse Hankel transform is replaced 


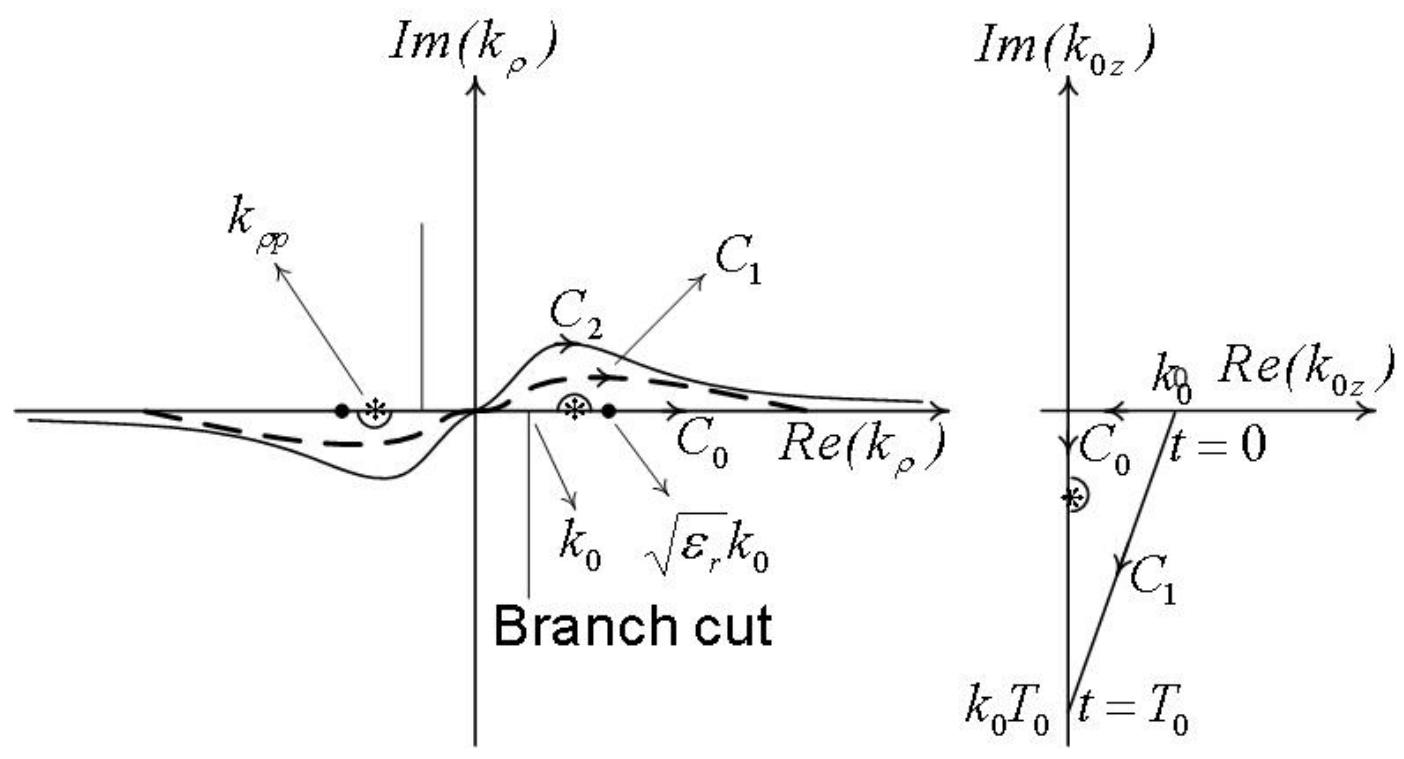

Figure 4.3: The integration paths of inverse Hankel transform in both $k_{\rho}$ and $k_{0 z}$ planes for a grounded dielectric slab

by a finite integral, no significant error is expected. The infinite and truncated integration paths are shown in Fig. 4.3 for a single layer structure (grounded dielectric slab). The straight line has a parametric equation of:

$$
C_{1}: k_{s z}=k_{s}\left[-j t+1-\frac{t}{T_{0}}\right] \quad 0 \leqslant t \leqslant T_{0}
$$

where $t$ is the running parameter and $T_{0}$ is the truncation point. $T_{0}$ should be chosen bigger than the largest $\sqrt{\varepsilon_{r}}$. Hence there is one-to-one correspondence between the running parameter and $k_{s z}$ on $C_{1}$, which means that $\tilde{G}_{c i}$ is a complex function of a real variable of a finite range on $C_{1}$ and therefore Prony's method can be used to find $a_{i}$ 's and $b_{i}$ 's in 4.174. [92]. The spatial $\tilde{G}_{c i}$ can be obtained as [68]:

$$
G_{c i}=\frac{1}{4 \pi} \sum_{i=1}^{N_{c i}} a_{i} \frac{e^{-j k_{s} \sqrt{\rho^{2}-b_{i}^{2}}}}{\sqrt{\rho^{2}-b_{i}^{2}}}
$$

As can be seen, a closed-form expression has been given for the spatial Green's function consisting of three parts, i.e. quasi-static images, surface-waves, and complex images. 
CHAPTER 4. COMPLEX IMAGES METHOD

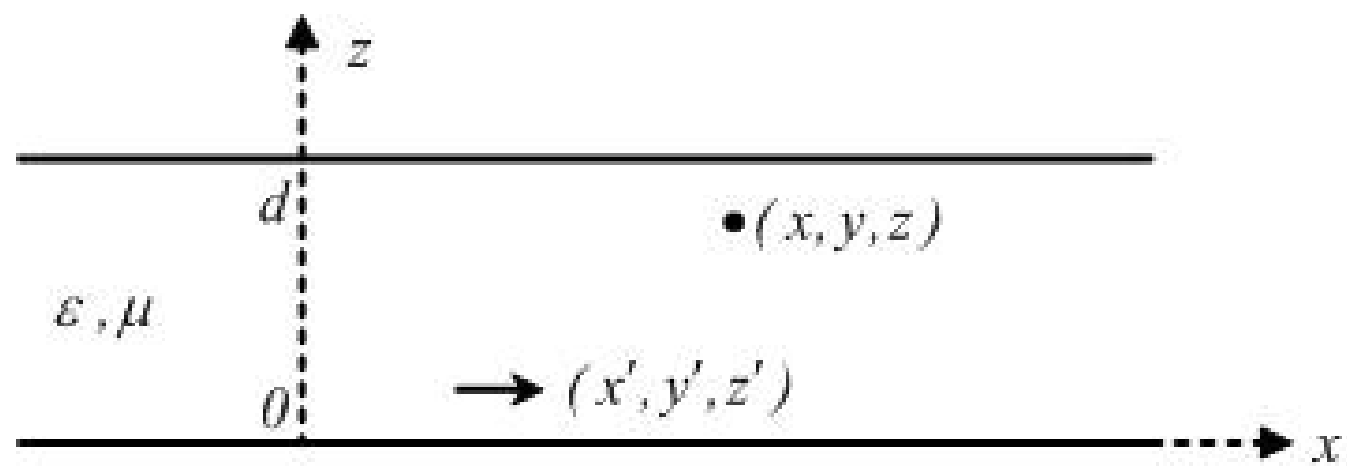

Figure 4.4: An $x$-directed horizontal magnetic dipole in a homogeneous parallelplate waveguide

We have developed computer programs for both an HED and an HMD embedded in the structure shown in Fig. 4.2. This is part of the future work of this thesis that will be discussed in the final chapter.

\subsubsection{Complex Images for an HMD in a Parallel-Plate Waveguide}

Though the problem of finding fields due to a horizontal electric or magnetic dipole in a parallel-plate waveguide (PPW) seems to be a trivial task, the conventional solutions in terms of image and modal expansions are only convergent in their respective regions. A "relay race" scheme of image and modal expansions could have been used [57], but since our intention was to extend the applicability of the network model to multi-layer planar circuits where each dielectric could be multilayered as well, a unique approach seemed to be necessary. Complex images method has been successfully applied to the problem of an HED in both homogeneous and layered parallel-plate waveguides [57]. With some modifications the same procedure can be followed for an HMD in a parallel-plate waveguide [93].

Fig. 4.4 shows an $x$-directed Horizontal Magnetic Dipole (HMD) in a homogeneous PPW. Spatial expressions for the vector and scalar potential Green's functions can be obtained by the taking the inverse Hankel transforms of the spectral 


\section{CHAPTER 4. COMPLEX IMAGES METHOD}

expressions [78] as:

$$
4 \pi G_{F}^{x x}=4 \pi \mu G_{q, m}^{x}=\int_{-\infty}^{+\infty} \frac{\tilde{T}_{T M}(z)}{j 2 k_{z}} H_{0}^{(2)}\left(k_{\rho} \rho\right) k_{\rho} d k_{\rho}
$$

where $\tilde{T}_{T M}$ is the transmission coefficient from source plane to field plane and can be derived in terms of the Fresnel reflection coefficients from 4.167a-4.168c as:

$$
\tilde{T}_{T M}\left(z, z^{\prime}\right)=\frac{\left(1+e^{-j 2 k_{z} z^{\prime}}\right)\left(1+e^{-j 2 k_{z}(d-z)}\right) e^{-j k_{z}\left(z-z^{\prime}\right)}}{1-e^{-j 2 k_{z} d}} \text { for } z>z^{\prime}
$$

A similar expression can be obtained for $z<z^{\prime}$ simply by exchanging the locations of $z$ and $z^{\prime}$. The solution in terms of real images can be obtained in the form of an infinite series by either adding the contributions of the source and each of its images or equivalently by expanding $\tilde{T}_{T M}$ into Taylor series:

$$
\begin{array}{r}
\tilde{T}_{T M}\left(z, z^{\prime}\right)=\sum_{n=0}^{\infty}\left[e^{-j k_{z}\left(z-z^{\prime}+2 n d\right)}+e^{-j k_{z}\left(z+z^{\prime}+2 n d\right)}+e^{j k_{z}\left(z+z^{\prime}-2(n+1) d\right)}+\right. \\
\left.+e^{j k_{z}\left(z-z^{\prime}-2(n+1) d\right)}\right]
\end{array}
$$

The spatial expression for the image series can be obtained by substituting 4.179 into 4.177 and using Sommerfeld identity:

$$
4 \pi G_{F}^{x x}=4 \pi \mu G_{q}=\sum_{n=0}^{\infty}\left[\frac{e^{-j k r_{n 1}}}{r_{n 1}}+\frac{e^{-j k r_{n 2}}}{r_{n 2}}+\frac{e^{-j k r_{n 3}}}{r_{n 3}}+\frac{e^{-j k r_{n 4}}}{r_{n 4}}\right]
$$

where

$$
\begin{aligned}
& r_{n 1}=\sqrt{\rho^{2}+\left(z-z^{\prime}+2 n d\right)^{2}} \\
& r_{n 2}=\sqrt{\rho^{2}+\left(z+z^{\prime}+2 n d\right)^{2}} \\
& r_{n 3}=\sqrt{\rho^{2}+\left(z+z^{\prime}-2(n+1) d\right)^{2}} \\
& r_{n 4}=\sqrt{\rho^{2}+\left(z-z^{\prime}-2(n+1) d\right)^{2}}
\end{aligned}
$$

Modal expansion can also be obtained from 4.178 by rearranging $\tilde{T}_{T M}$ into the following form:

$$
\frac{\tilde{T}_{T M}(z)}{j 2 k_{z}}=-\frac{1}{2 k_{z}} \cot \left(\frac{k_{z} d}{2}\right) \frac{\cos \left(k_{z} z^{\prime}\right) \cos \left[k_{z}(d-z)\right]}{\cos ^{2}\left(k_{z} d / 2\right)}
$$




\section{CHAPTER 4. COMPLEX IMAGES METHOD}

and then using Mittag-Leffler theorem for the cotangent factor [94] to obtain:

$$
\frac{\tilde{T}_{T M}(z)}{j 2 k_{z}}=-\frac{1}{2 k_{z}} \frac{\cos \left(k_{z} z^{\prime}\right) \cos \left[k_{z}(d-z)\right]}{\cos ^{2}\left(k_{z} d / 2\right)}\left\{\frac{2}{k_{z} d}+\frac{4 k_{z}}{d} \sum_{n=1}^{\infty} \frac{1}{k_{z}^{2}-(2 n \pi / d)^{2}}\right\}
$$

The spatial expression can be found by substituting 4.183 into 4.177 and using the residue theorem to calculate the integrals [95]:

$$
4 \pi G_{F}^{x x}=4 \pi \mu G_{q}=-\frac{\pi j}{d} H_{0}^{(2)}(k \rho)-\frac{2 \pi j}{d} \sum_{n=1}^{\infty} \cos \left(\frac{2 n \pi z^{\prime}}{d}\right) \cos \left(\frac{2 n \pi z}{d}\right) H_{0}^{(2)}\left(k_{\rho p} \rho\right)
$$

where $k_{\rho p}^{2}=k^{2}-(2 n \pi / d)^{2}$. The expression 4.184 represents the modal expansion of the potentials excited by an HMD [57]. Obviously, only the terms with $k>2 n \pi / d$ can propagate and all other modes are below cutoff and correspond to evanescent modes. The conspicuous difference between 4.184 and the corresponding equation for an HED in [57] stems from the fact that the fundamental mode excited by an HMD has a cutoff frequency of zero and hence can always propagate. As will be seen shortly, the method proposed in [57] cannot be directly applied to the problem at hand and needs minor adjustments.

A third approach for calculating vector and scalar potentials and hence fields has been proposed in [57]. This approach is based on complex images method and utilizes the merits of both image and modal expansion. We will show that modal expansion converges very slowly when the observation point is close to the source. On the other hand, image series converges very slowly for large distances. In the proposed method, $\tilde{T}_{T M}(z)$ is written as follows:

$$
\tilde{T}_{T M}=e^{-j k_{z}\left(z-z^{\prime}\right)}+j 2 k_{z} P_{m}+\left(\tilde{T}_{T M}-e^{-j k_{z}\left(z-z^{\prime}\right)}-j 2 k_{z} P_{m}\right)
$$

where $P_{m}$ is part of the modal expansion that only includes propagating modes, i.e. the fundamental mode and the modes with $k>2 n \pi / d$. The first term in 4.185 is the direct term due to the HMD in a homogeneous medium. The first two terms have analytical inverse Hankel transforms. A closed-form expression in spatial domain corresponding to the third term can be found using complex images method. Consequently, closed-form expression for the spatial vector and scalar potentials takes the following form:

$$
4 \pi G_{F}^{x x}=4 \pi \mu G_{q}=G_{0}+G_{c i}+G_{p_{m}}
$$

where

$$
G_{0}=\frac{e^{-j k r_{0}}}{r_{0}}, \quad r_{0}=\sqrt{\rho^{2}+\left(z-z^{\prime}\right)^{2}}
$$


CHAPTER 4. COMPLEX IMAGES METHOD

$$
\begin{gathered}
G_{c i}=\sum_{i=1}^{N_{c i}} a \frac{e^{-j k r_{i}}}{r_{i}}, \quad r_{i}=\sqrt{\rho^{2}+\left(-j b_{i}\right)^{2}} \\
G_{p_{m}}=-\frac{\pi j}{d} H_{0}^{(2)}(k \rho)-\frac{2 \pi j}{d} \sum_{n=1}^{N_{\text {propag }}} \cos \left(\frac{2 n \pi z^{\prime}}{d}\right) \cos \left(\frac{2 n \pi z}{d}\right) H_{0}^{(2)}\left(k_{\rho p} \rho\right)
\end{gathered}
$$

where $N_{\text {propag }}$ is the number of propagating modes, excluding the fundamental mode. $N_{c i}$ is chosen in the range $3-5$ as recommended in [57].

As it will be demonstrated in the next section, we found out that subtracting propagating modes from $\tilde{T}_{T M}$ and then applying complex images method was not always beneficial. On the contrary to the HED case, at close distances no propagating mode should be taken into account [93]. 
CHAPTER 4. COMPLEX IMAGES METHOD

\subsubsection{Numerical Results for Fields and Potentials of an HMD in a Parallel-Plate Waveguide Using Complex Images}

With reference to Fig. 4.4, we consider a parallel-plate waveguide with $d=0.2 \lambda_{0}$, $z=z^{\prime}=0$, filled with air, i.e. $\varepsilon=\varepsilon_{0}$ and $\mu=\mu_{0} . \lambda_{0}$ is the wavelength in dielectric substrate, which is air in this case. all dimensions and distances are normalized with respect to $\lambda_{0}$, so frequency need not be specified. Only the fundamental mode is supported by this structure. We consider an $x$-directed surface magnetic current distribution as follows:

$$
\vec{M}=\hat{x} M=\hat{x} \frac{h-|x|}{h w_{s}}
$$

which has the form of rooftop basis functions used to expand the equivalent magnetic current over the slot. Rooftop basis functions are triangular in longitudinal direction and uniform in transverse direction [96]. $2 h=\lambda_{0} / 50$ is the support of the basis functions in $x$-direction. $w_{S}=\lambda_{0} / 100$ is the support of the distribution in $y$-direction. Rooftop basis functions are commonly used in method of moments. This choice has some practical advantages which has been discussed in detail in the literature [97], [98], [99], [100]. A brief explanation is also provided in Appendix C.

In MPIE formulation the fields produced by this equivalent magnetic current can be found from vector and scalar potentials. We are only interested in transverse magnetic fields in a parallel-plate waveguide (Fig. 4.4) which can be given as:

$$
\begin{aligned}
& H_{x}(x, y)=-j \omega\left[\varepsilon G_{F}^{x x} * M+\frac{1}{\omega^{2}} \frac{\partial G_{q, m}^{x}}{\partial x} * \frac{\partial M}{\partial x_{0}}\right] \\
& H_{y}(x, y)=\frac{1}{j \omega} \frac{\partial G_{q, m}^{x}}{\partial y} * \frac{\partial M}{\partial x_{0}}
\end{aligned}
$$

where $*$ is the convolution operator defined as:

$$
G_{F}^{x x} * M=\int G_{F}^{x x}\left(x-x_{0}, y-y_{0}\right) M\left(x_{0}, y_{0}\right) d x_{0} d y_{0}
$$

In complex images method spatial Green's functions consist of exponential and Hankel function terms. For polynomial expansion functions some of the convolution integrals can be evaluated analytically, whereas some can be converted to one dimensional integrals which can be computed numerically. This has already been done for exponential terms [101], [96]. We have implemented the same approach 
CHAPTER 4. COMPLEX IMAGES METHOD

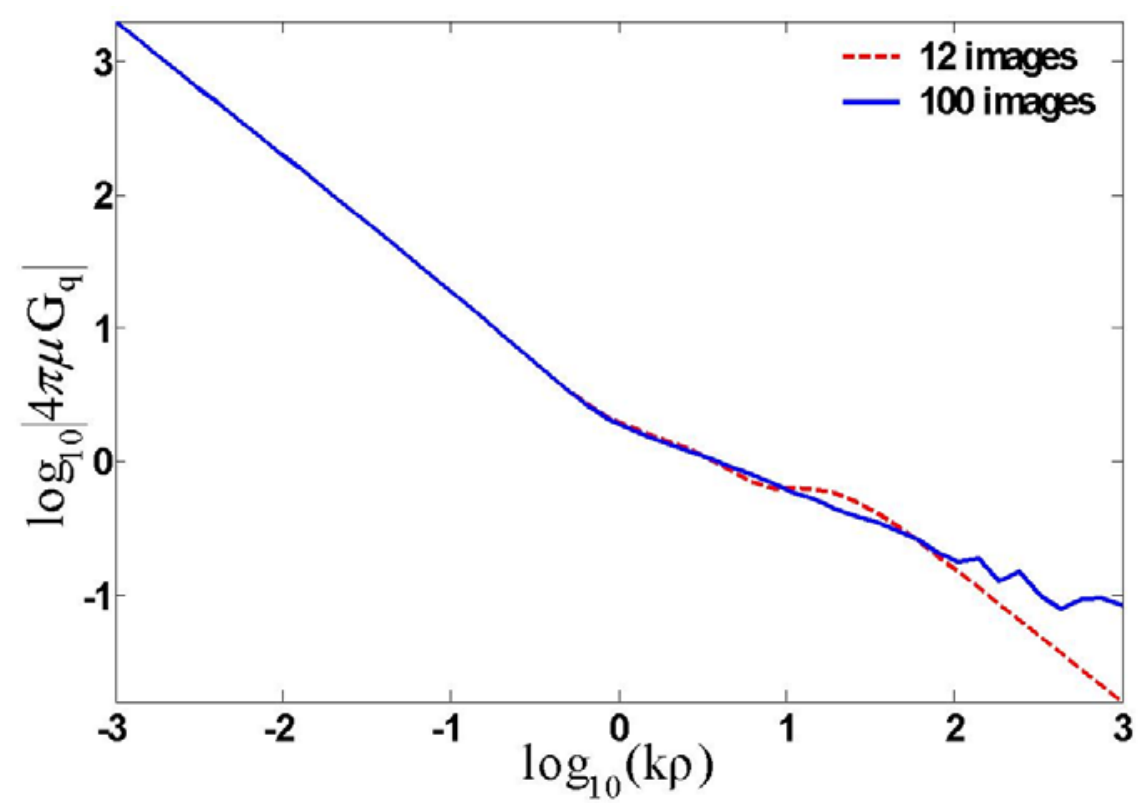

Figure 4.5: Amplitude of $4 \pi \mu G_{q}$ from image expansion for $d=0.2 \lambda_{0}$ and $z=z^{\prime}=0$.

for Hankel function terms (Appendix C). However, it was observed that calculating contribution of exponential terms separately didn't yield satisfactory results at large distances. This is due to the fact that complex images have imaginary spatial locations and produce highly oscillatory fields. Therefore, potentials were divided into two parts. One part included all exponential terms (complex images and the direct term) and the second part all propagating modes. The contribution of the first part was calculated as an entire entity numerically, whereas the second part was further broken down into modes and contribution of each mode was calculated separately using a combination of numerical and analytical integrations.

The Green's function calculated by image and modal expansions as well as their comparison to the results obtained by the method proposed in [57] are shown in Fig. 4.5, Fig. 4.6, and Fig. 4.7, respectively. As can be seen, from Fig. 4.5, for distances up to $\rho / \lambda_{0} \leq 0.16$, the number of real images can be as low as 12 . As we move farther away from the source, more images must be considered. But this in turn makes the results oscillatory. For example, with 100 images, oscillations start around $\rho / \lambda_{0} \cong 1.6$. This will be shown to have destructive effect on the fields.

On the other hand, modal expansion converges very fast for $\rho / \lambda_{0} \geq 0.16$, Fig. 4.6. It seems that in most cases considering only the propagating modes of modal 
CHAPTER 4. COMPLEX IMAGES METHOD

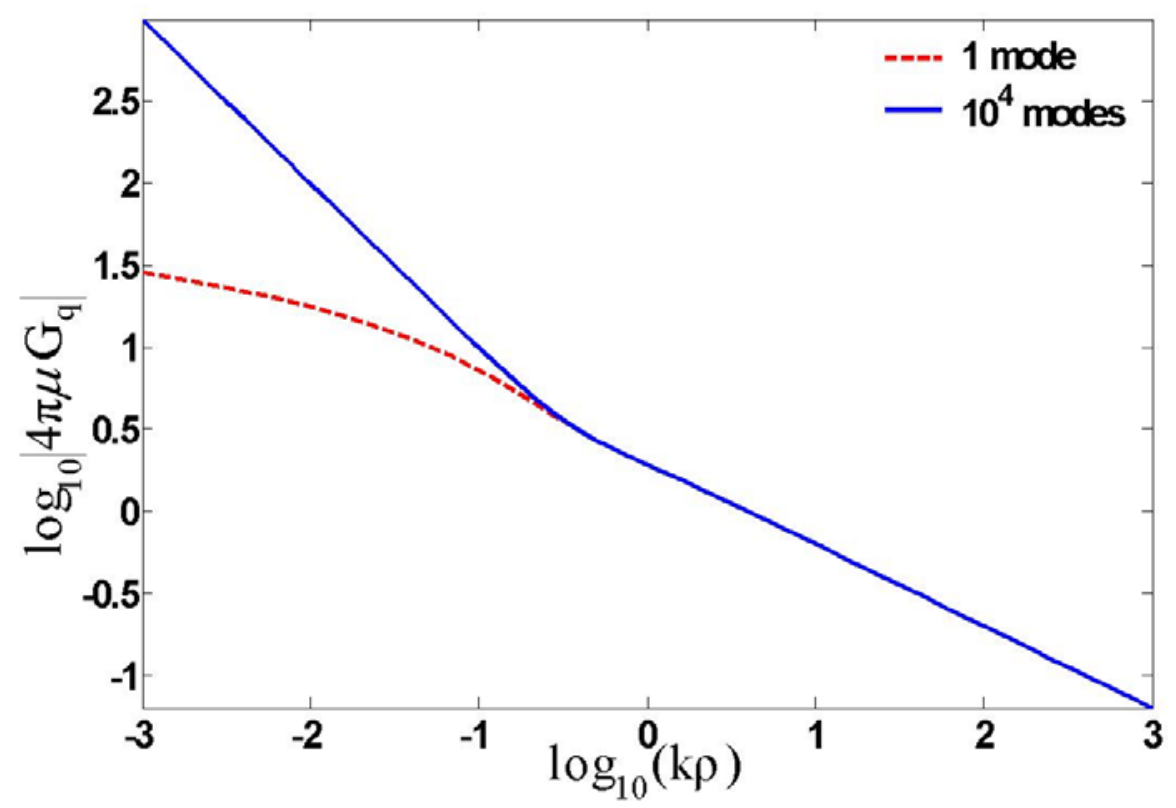

Figure 4.6: Amplitude of $4 \pi \mu G_{q}$ from modal expansion for $d=0.2 \lambda_{0}$ and $z=z^{\prime}=$ 0 .

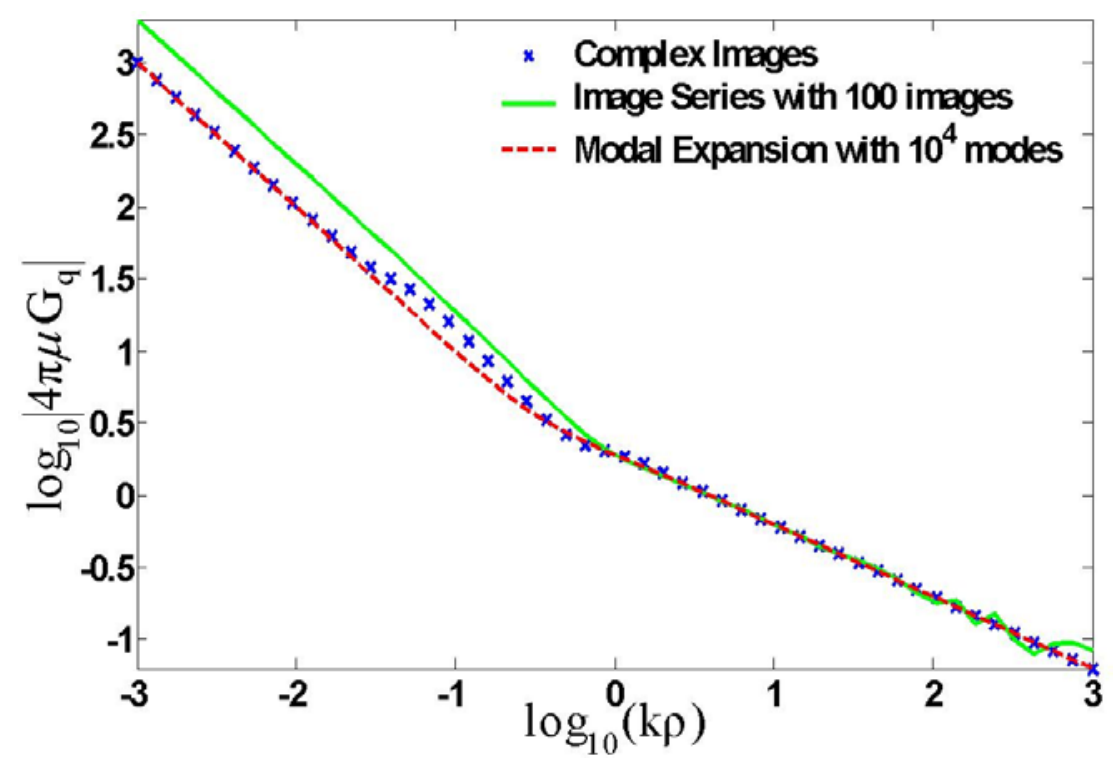

Figure 4.7: Amplitude of $4 \pi \mu G_{q}$ from image and modal expansions as well as complex images for $d=0.2 \lambda_{0}$ and $z=z^{\prime}=0$. 


\section{CHAPTER 4. COMPLEX IMAGES METHOD}

expansion is enough for large distances. However, for $\rho / \lambda_{0} \leq 0.16$, convergence was extremely slow and was not achieved with even $10^{4}$ modes.

Fig. 4.7 shows the results from all three methods. As can be seen, complex images method diverges from image expansion for $\rho / \lambda_{0} \leq 0.16$. This is similar to the issue discussed in [88] and can be attributed to the presence of the fundamental mode. This can be verified by setting $P_{m}=0$ in 4.185 , i.e. ignoring any propagating modes and applying complex images method. The result is shown in Fig. 4.8 and compared with the results from the original method proposed in [57]. It can be concluded that for close distances, $P_{m}$ should not be subtracted from $T_{T M}$, while for large distances subtracting the contribution of propagating modes is necessary. This can be seen from Fig. 4.9 which compares all four approaches. It can also be observed that the two approaches for implementing complex images method coincide for $0.16 \leq \rho / \lambda_{0} \leq 1.6$. Therefore, no sharp boundary between the two approaches exists. However, after running some examples and comparing fields as well as the potentials, we found out that for the problem at hand $\rho / \lambda_{0}=1.6$ was a superior choice.

The transverse fields calculated from image and modal expansions and our proposed approach for the same structure are shown in Fig. 4.10 and Fig. 4.11. As can be seen, complex images method agrees quite well with image and modal expansions in their respective convergence zones. The $x$-directed magnetic current density had a distribution similar to 4.190 with $h=w_{S}=\lambda_{0} / 100$. In this case, no propagating mode is considered for $\rho / \lambda_{0} \leq 1.6$. It is also important to notice that $H_{x}$ from image expansion becomes highly oscillatory after $\rho / \lambda_{0}=1.6$. 
CHAPTER 4. COMPLEX IMAGES METHOD

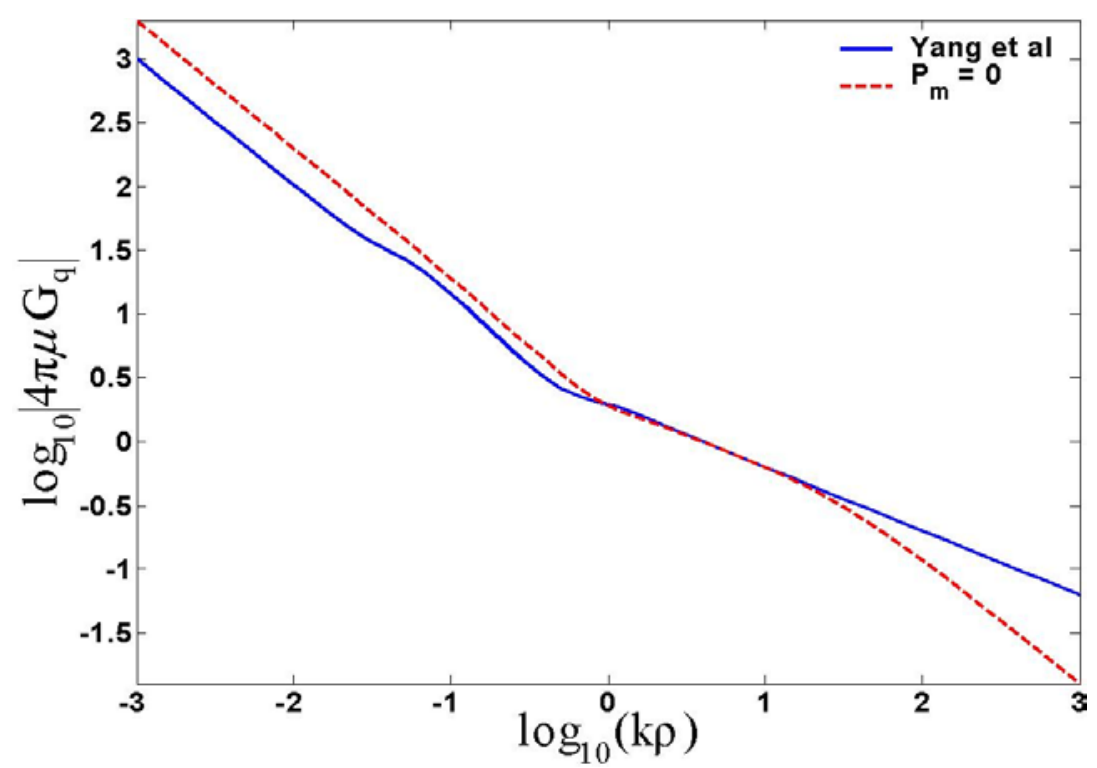

Figure 4.8: Amplitude of $4 \pi \mu G_{q}$ from complex images method for $d=0.2 \lambda_{0}$ and $z=z^{\prime}=0$ : with (-) and without (--) fundamental mode extracted.

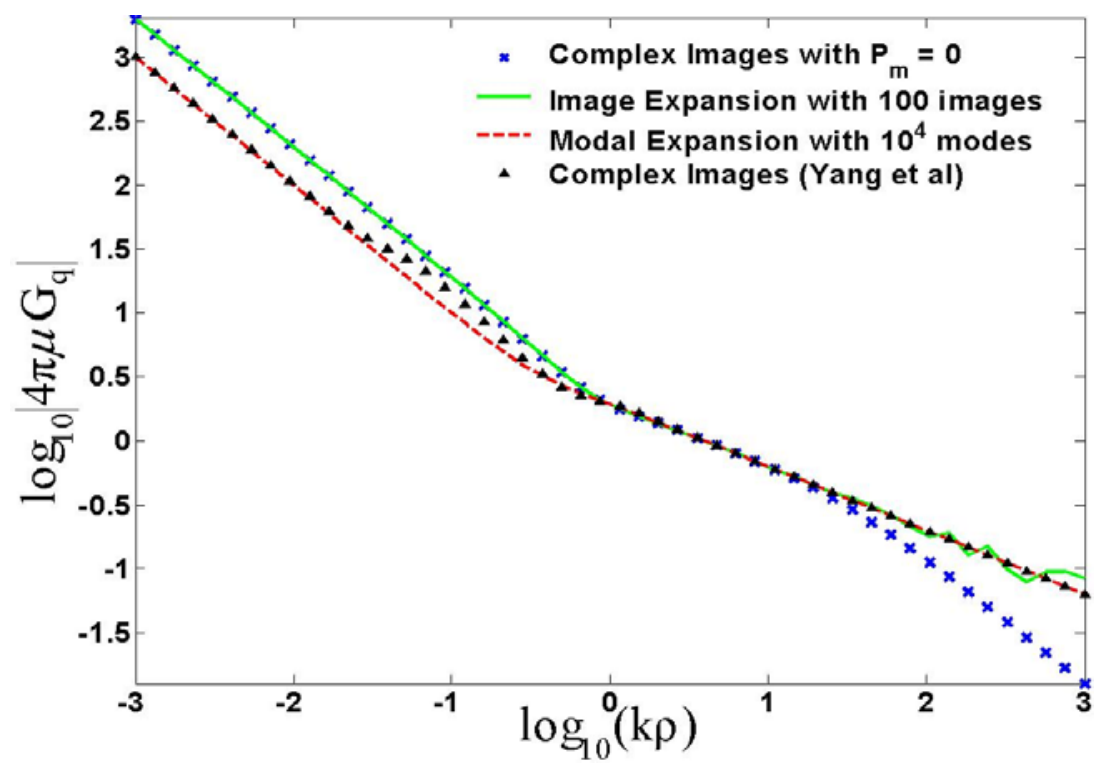

Figure 4.9: Amplitude of $4 \pi \mu G_{q}$ from image (-)and modal (--) expansions as well as complex images both with $(\Delta)$ and without $(\times)$ extracting propagating modes for $d=0.2 \lambda_{0}$ and $z=z^{\prime}=0$. 
CHAPTER 4. COMPLEX IMAGES METHOD

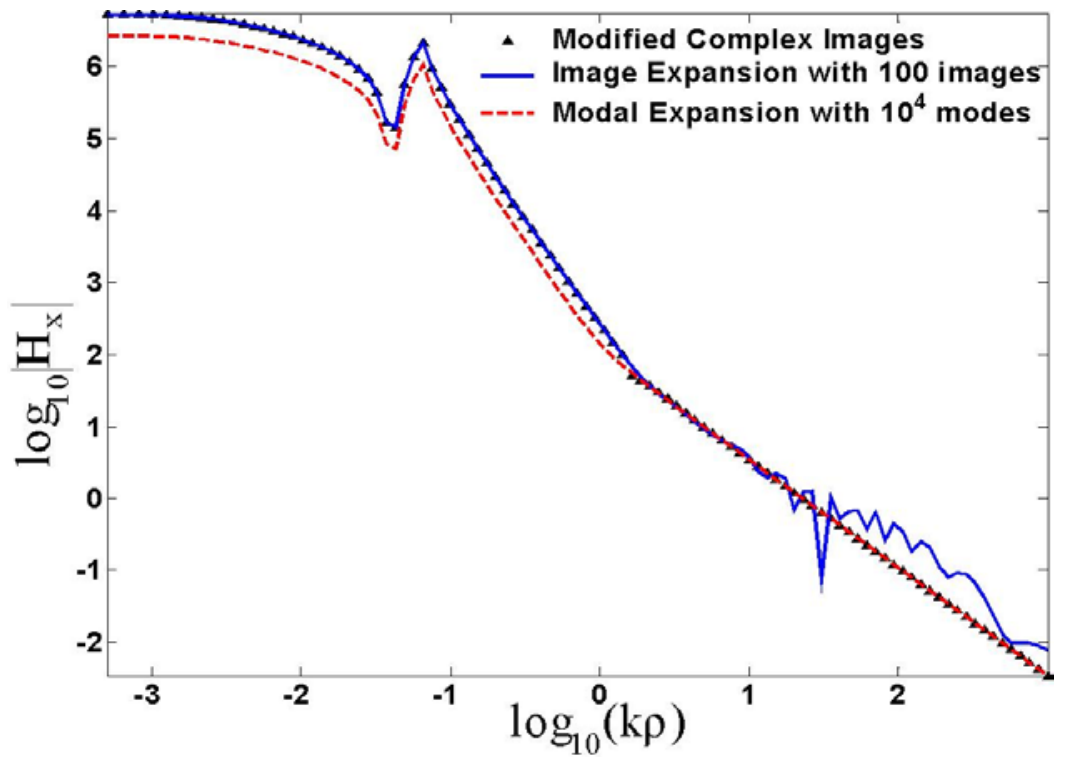

Figure 4.10: Amplitude of $H_{x}$ from image (-)and modal (--) expansions as well as complex images $(\Delta)$ for $d=0.2 \lambda_{0}$ and $z=z^{\prime}=0$.

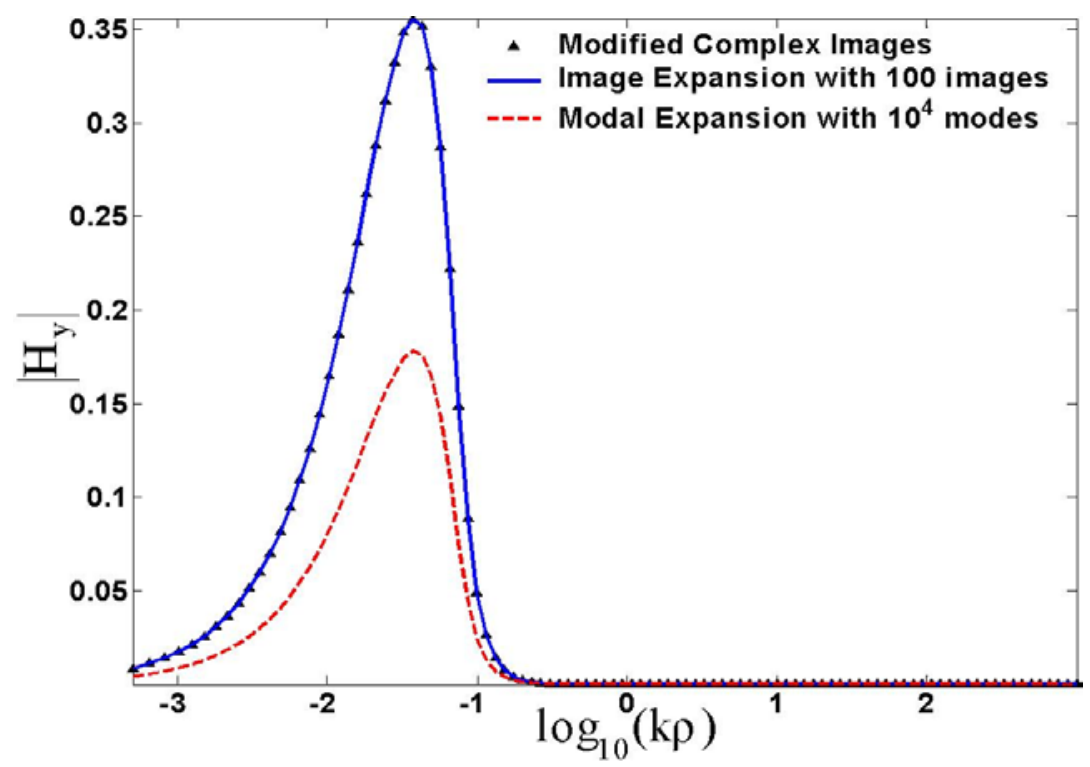

Figure 4.11: Amplitude of $H_{y}$ from image (-)and modal (--) expansions as well as complex images $(\Delta)$ for $d=0.2 \lambda_{0}$ and $z=z^{\prime}=0$. 


\section{Chapter 5}

\section{Numerical Results and Discussion}

In this chapter numerical results obtained from our method for some sample structures will be compared with the results from ANSOFT HFSS. In all examples, the structure consists of two metallic patches etched on two lossless dielectric slabs of dielectric constant $\varepsilon_{r}=9$ and thickness $d=0.38 \mathrm{~mm}$ on two sides of a common ground plane. The two circuits, however, are coupled through a narrow rectangular slot of length $L_{s}$ and width $w_{s}$ in their common ground plane. At $5 \mathrm{GHz}$ the slot is $\lambda / 2.5$ long and $\lambda / 40$ wide, where $\lambda$ is the wavelength in dielectric substrate. In this case the electric field within the slot will be in the transverse direction of the slot and consequently the equivalent magnetic current in the longitudinal direction.

Let $\hat{u}$ and $\hat{v}$ denote unit vectors in the longitudinal and transverse directions of the slot, respectively. Since the slot is narrow, the equivalent magnetic current over the slot is expanded in rooftop basis functions, which are triangular in longitudinal direction and uniform in transverse direction [96]:

$$
\vec{M}_{\ell}=\hat{u} M_{\ell}=\hat{u} \frac{h-\left|u-u_{\ell}\right|}{h w_{s}}
$$

where as before $2 h$ and $w_{s}$ are the supports of the basis functions in $x-$ and $y-$ directions, respectively. $u_{\ell}$ is the center point of the $\ell^{t h}$ basis function. These center points are uniformly spaced along the slot.

\subsubsection{Single-pole two-layer filter}

First we consider two rectangular patches coupled through a narrow rectangular slot. This structure can be considered a single pole two-layer filter. Each patch is 


\section{CHAPTER 5. NUMERICAL RESULTS AND DISCUSSION}

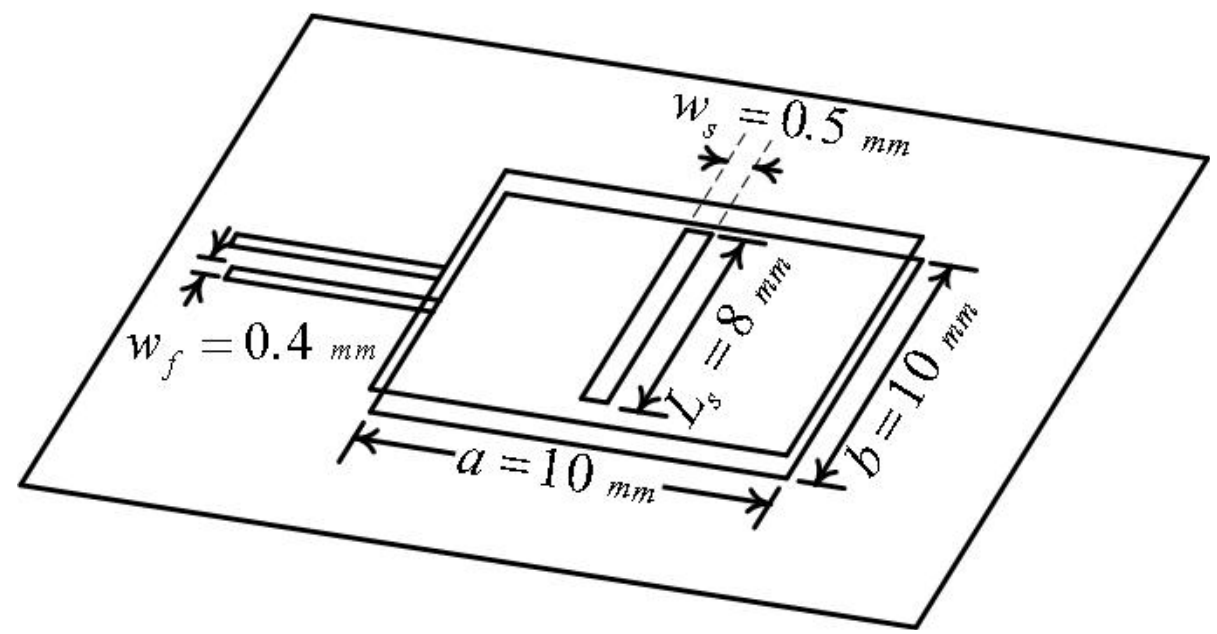

Figure 5.1: Two-layer single pole filter

fed by a $50 \Omega$ microstrip line of width $w_{f}=0.4 \mathrm{~mm}$, as shown in Fig. 5.1. Both patches are squares with dimensions $a=b=10 \mathrm{~mm}$. No loss is considered, but in order to account for the energy stored in fringing fields, sides are extended by [37]:

$$
\Delta \ell=\frac{0.412 d\left(\varepsilon_{r}+0.3\right)(a / d+0.246)}{\left(\varepsilon_{r}-0.258\right)(a / d+0.8)}
$$

Fig. 5.2 and Fig. 5.3 show the comparison of magnitudes of $S_{11}$ and $S_{21}$, respectively. Also shown in these figures are the results from generalized MNM for regular shapes. The predicted resonance frequency by generalized MNM is $4.96 \mathrm{GHz}$, which is very close to $4.95 \mathrm{GHz}$ from both HFSS and modal expansion. The error in resonance frequency is only $0.2 \%$. Modal expansion predicts the resonance frequency with almost no error, but it's applicability is limited to regular shapes. In addition, it was observed that when the slot was not perpendicular to the input feedline, the modal expansion suffered from poor convergence. In conventional MNM convergence can be dramatically improved by converting the double summation to a single summation [102], but the same approach proved to be inapplicable in this case. 


\section{CHAPTER 5. NUMERICAL RESULTS AND DISCUSSION}

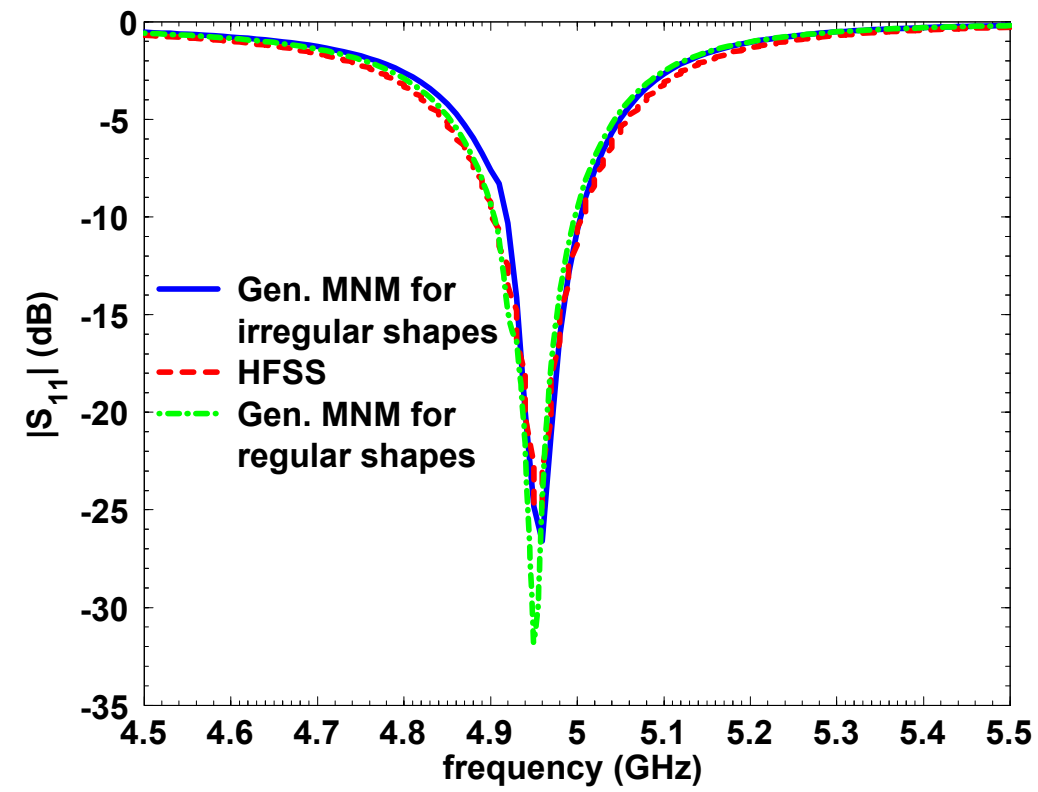

Figure 5.2: Return-loss of a single-pole two-layer filter

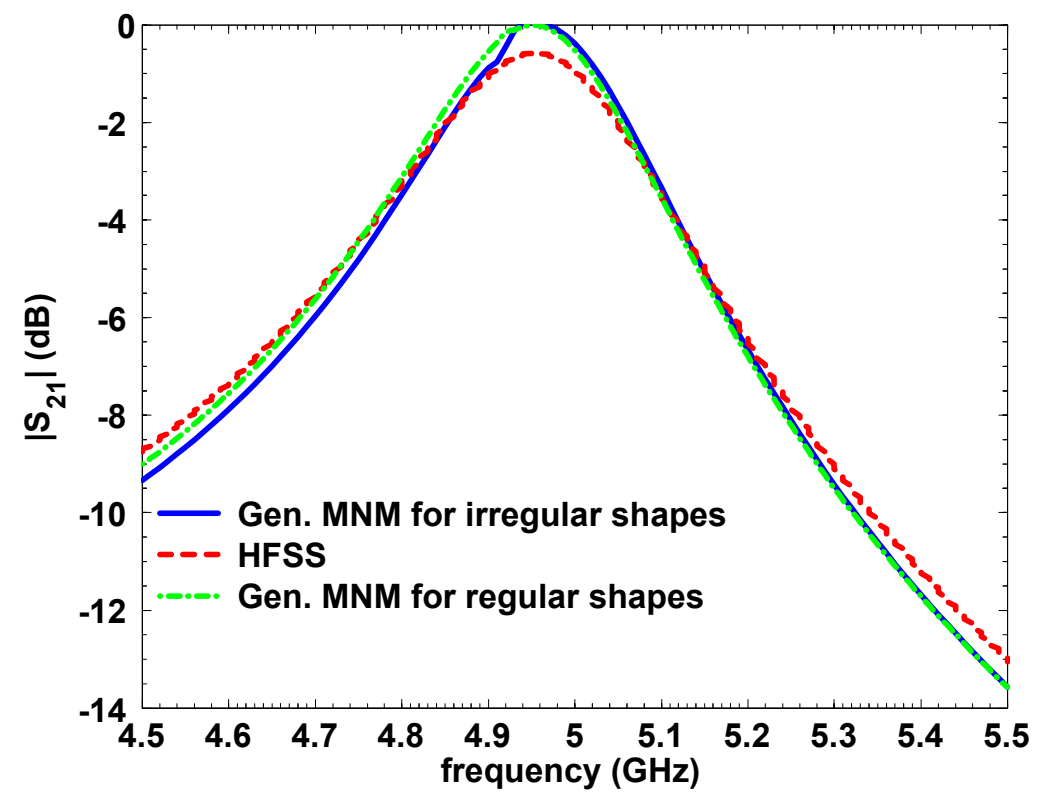

Figure 5.3: The amplitude of $S_{21}$ for the single-pole two-layer filter 


\section{CHAPTER 5. NUMERICAL RESULTS AND DISCUSSION}

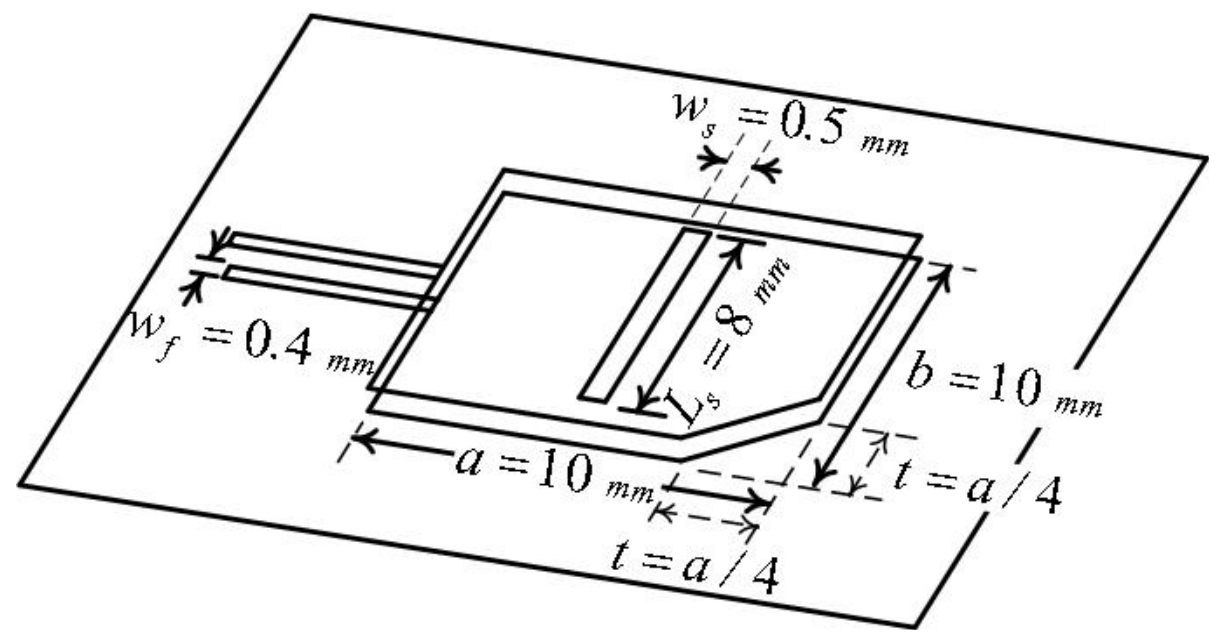

Figure 5.4: Two-layer dual-band filter

\subsubsection{Dual-Band Filter}

As a second example, we consider a two-layer dual-band filter comprising two identical corner-cut patches, as shown in Fig. 5.4. The patches are the same size as in the single-pole filter, with a symmetric right-angled triangular section measuring $t=a / 4$ on the sides cut out from one corner. As before, 5.2 has been used to account for the presence of fringing fields. Each of the corner cut patches is a dual-mode filter by itself, but dual-band performance can be achieved by utilizing two resonators coupled through a slot in ground plane. A corner cut patch does not have a canonical shape and therefore cannot be analyzed by modal expansion. Other analysis methods such as contour integral or desegmentation can, however, be used. Since our generalization of MNM method is based on contour integral equation, it can be used for analysis and design of a two-layer structure consisting of two corner cut patches. Comparison between the results for $\left|S_{11}\right|$ and $\left|S_{21}\right|$ from are method and the results from HFSS are shown in Fig. 5.5 and Fig. 5.6, respectively. As can be seen, the resonance frequencies predicted by our method are $4.92 \mathrm{GHz}$ and $5.19 \mathrm{GHz}$, versus $4.93 \mathrm{GHz}$ and $5.18 \mathrm{GHz}$, predicted by HFSS, an error of almost $0.2 \%$. The error in transmission zero is almost the same. Our method predicts the transmission zero to be at $5.03 \mathrm{GHz}$, very close to $5.04 \mathrm{GHz}$ predicted by HFSS. 
CHAPTER 5. NUMERICAL RESULTS AND DISCUSSION

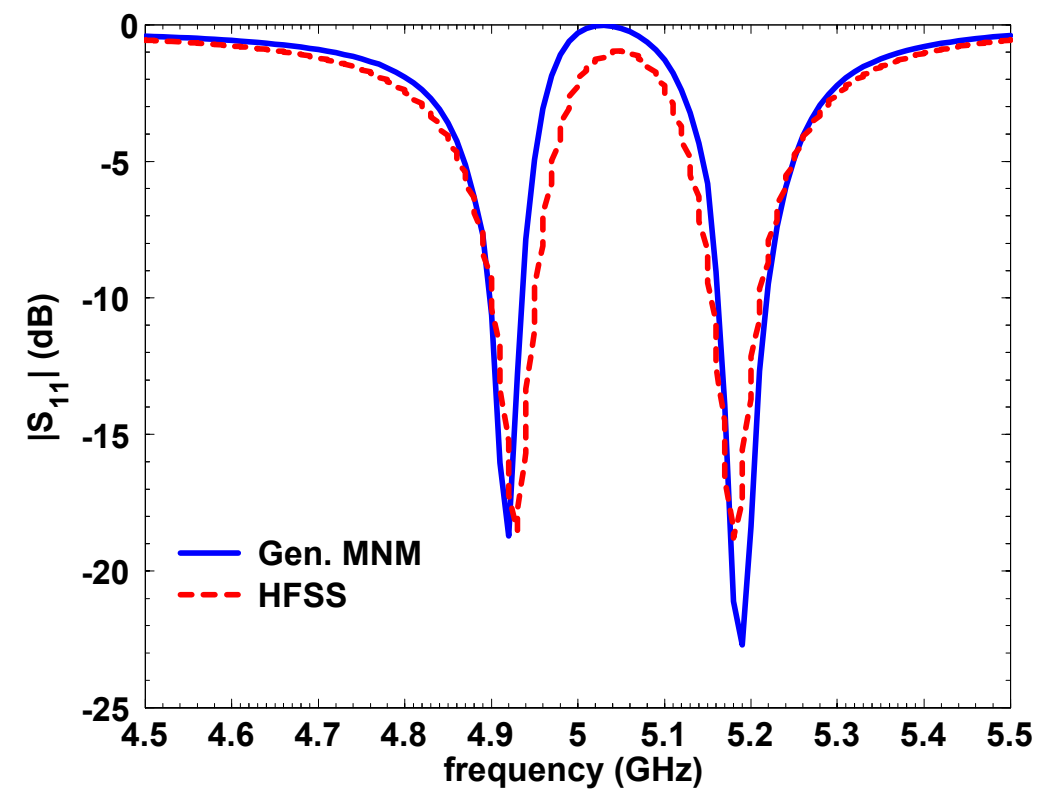

Figure 5.5: Return-loss of two-layer dual-band filter

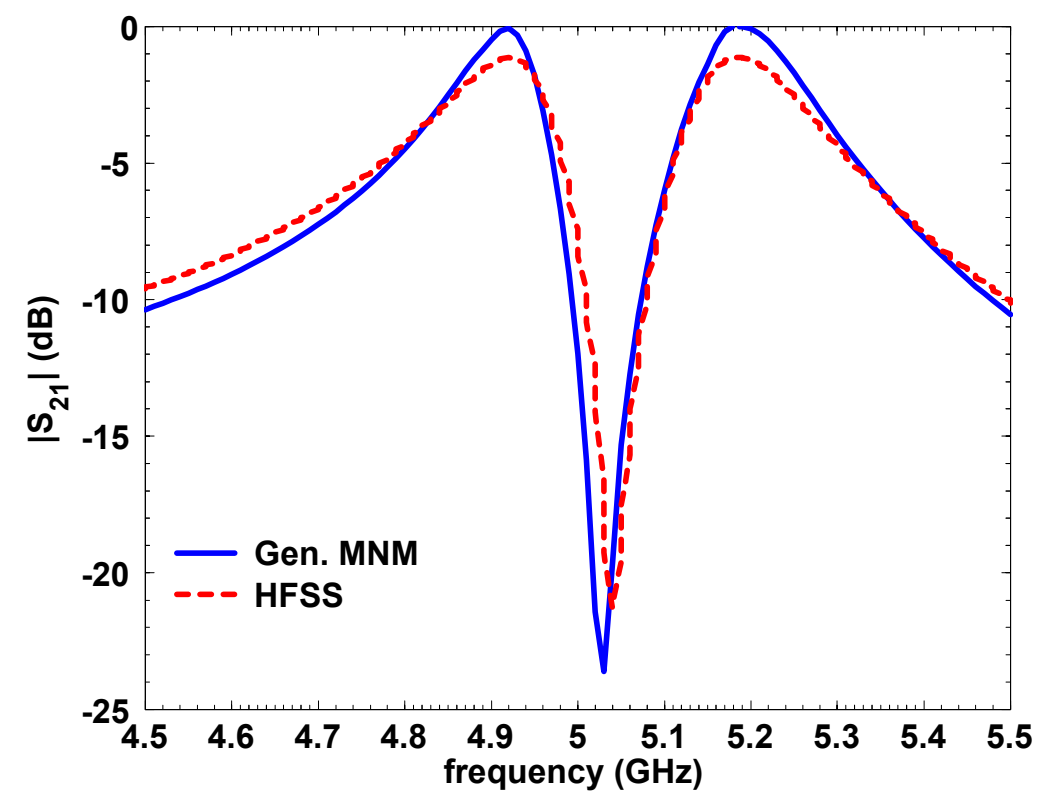

Figure 5.6: $\left|S_{21}\right|$ for two-layer dual-band filter 


\section{CHAPTER 5. NUMERICAL RESULTS AND DISCUSSION}

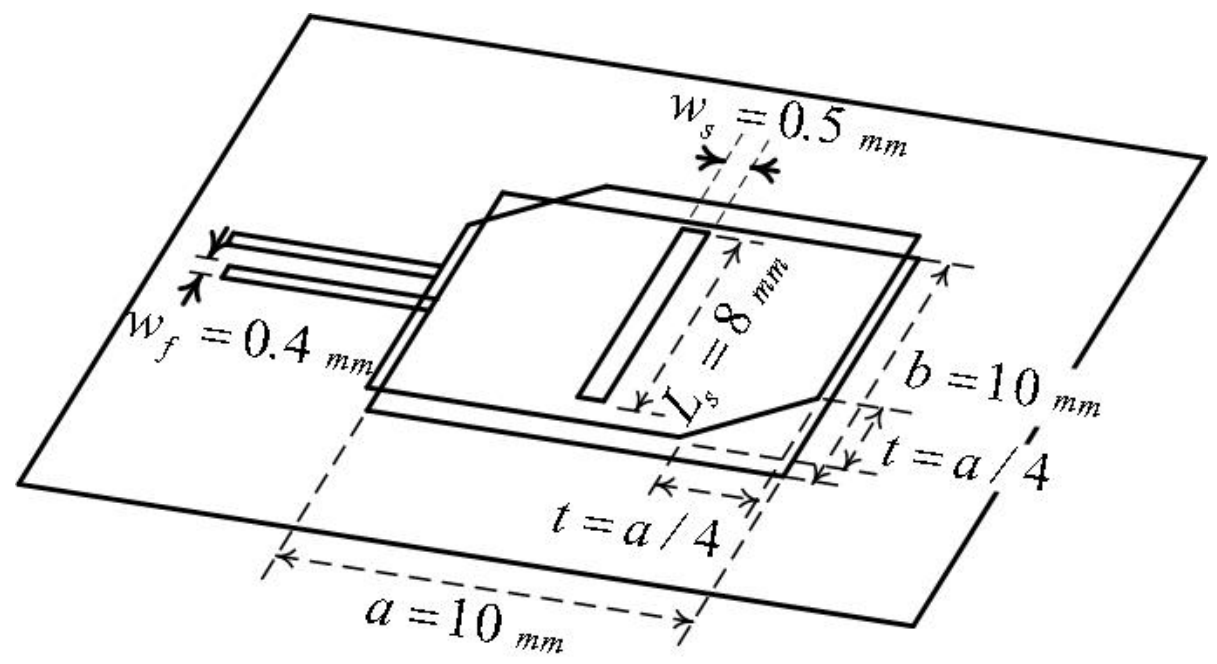

Figure 5.7: Multi-mode filter

\subsubsection{Multi-Mode Filter}

As the third example, we consider a square patch coupled to a double corner-cut patch, Fig. 5.7. The patches and the cutouts are both similar to the previous example. Port 1 refers to the microstrip line feeding the rectangular patch. In this case, the first resonance occurs at $4.92 \mathrm{GHz}$ for our method and $4.91 \mathrm{GHz}$ for HFSS, an error of almost $0.2 \%$. The second resonance, however, has an error of $0.56 \%$ (5.38 $\mathrm{GHz}$ from our method versus $5.35 \mathrm{GHz}$ from HFSS). The results are shown in Fig. 5.8 and Fig. 5.9. The discrepancy can be attributed to several factors, the most important being rather strong radiation at the second resonance which is due to the upper patch. There is a strong transmission zero at $5.15 \mathrm{GHz}(5.16 \mathrm{GHz}$ from our method, an error of less than $0.2 \%$ ), which can only be justified through cross-coupling of the modes. Hence, this structure can be considered a multi-mode filter. 
CHAPTER 5. NUMERICAL RESULTS AND DISCUSSION

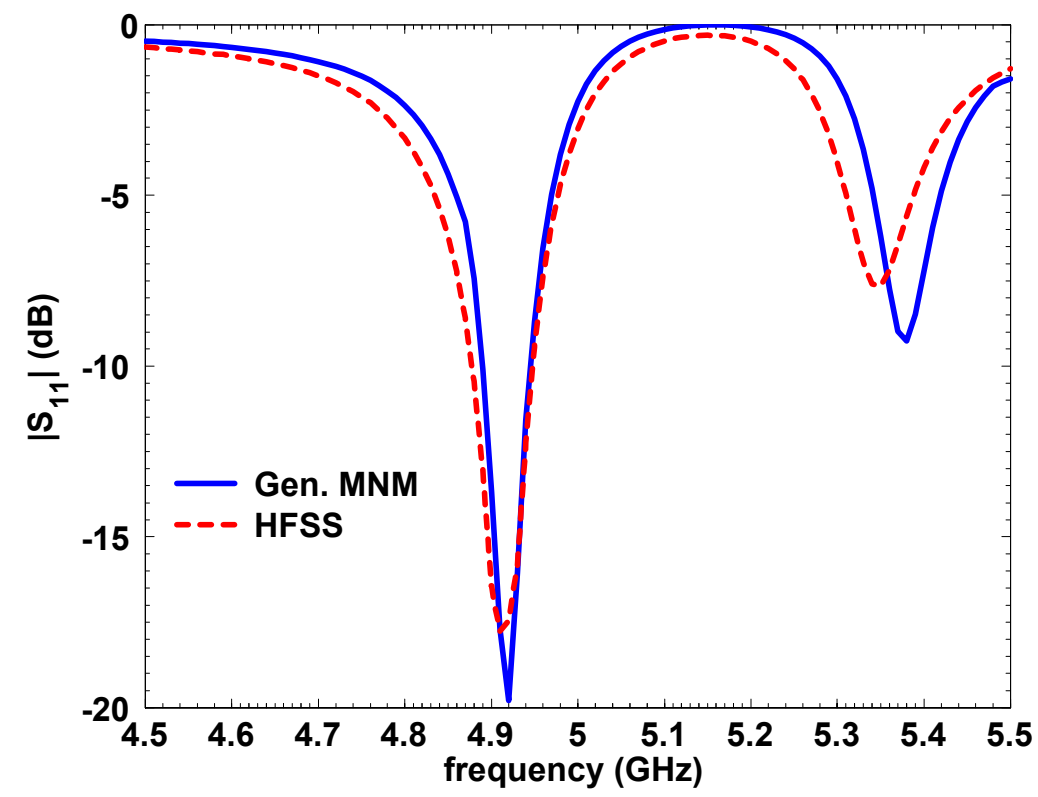

Figure 5.8: Return-loss for multi-mode filter

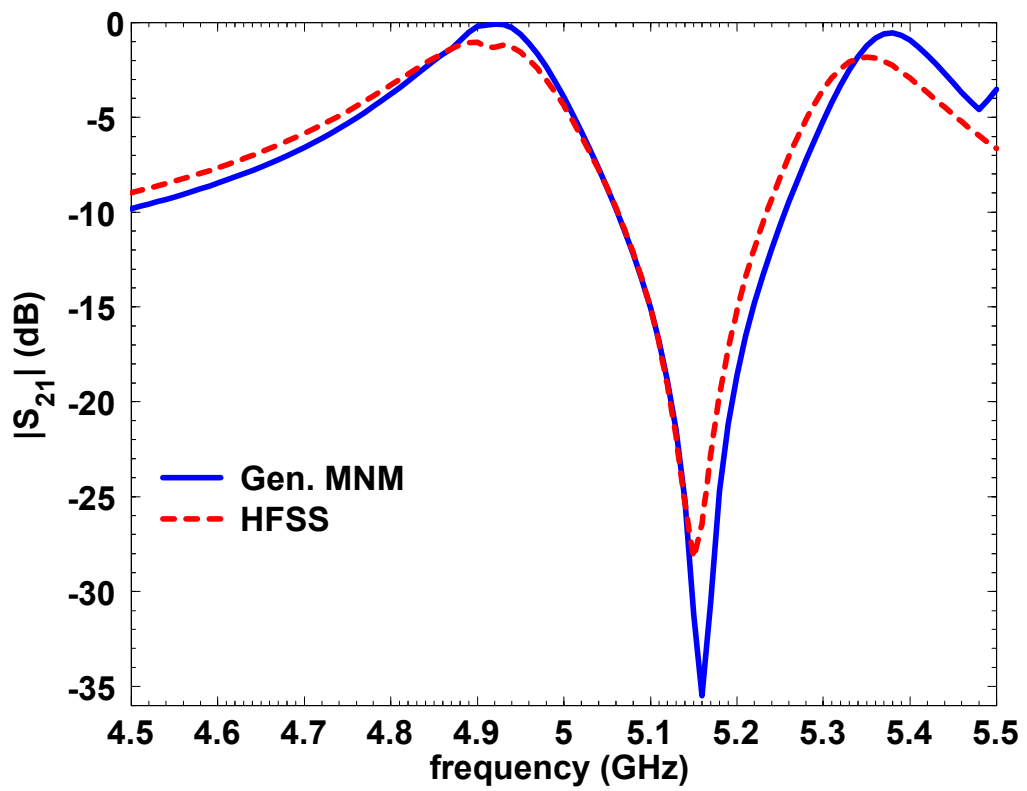

Figure 5.9: $\left|S_{21}\right|$ for multi-mode filter 


\section{CHAPTER 5. NUMERICAL RESULTS AND DISCUSSION}

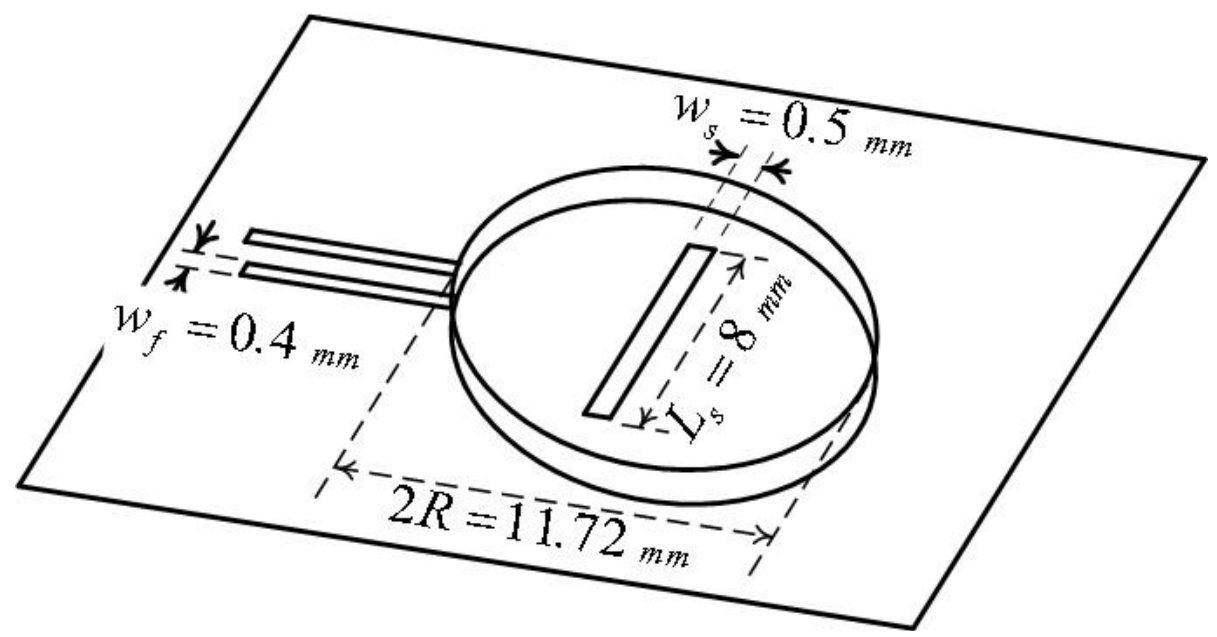

Figure 5.10: Two circular patches coupled through a slot in ground plane

\subsubsection{Circular Patches}

Circular patches are also very common both as resonators and as antennas. Here we have considered two circular patches coupled through a slot in their common ground planes, as shown in Fig. 5.10. Both patches have a radius of $R=5.86 \mathrm{~mm}$. In order to account for the fringing fields in this case, equation 5.2 has been used to calculate $\Delta \ell$ for $a=2 R$ and then extend the radius of the patch by $\Delta \ell / 2$. The reason is that the formula for calculating $\Delta \ell$ is only accurate when $a / d \gg 1$, otherwise it overestimates $\Delta \ell$. For example $\Delta \ell(R)=0.16 \mathrm{~mm}$, while $\Delta \ell(2 R)=0.163 \mathrm{~mm}$. Extending the radius by $\Delta \ell(R)$ means a $2.7 \%$ increase, which can shift the resonance frequency well below the real resonance frequency due to fringing fields, whereas extending the radius by $\Delta \ell(2 R) / 2$ will shift the resonance frequency just as much needed to account for fringing fields.

Besides contour integral equation, circular patches can also be analyzed using eigenfunction expansion. Return-loss and $\left|S_{21}\right|$ from generalized MNM using both contour integral and modal expansion as well as HFSS full-wave analysis are shown in Fig. 5.11 and Fig. 5.12, respectively. The resonance frequency from both modal analysis and contour integral method is $5.02 \mathrm{GHz}$ and from HFSS is $5.03 \mathrm{GHz}$, an error less than $0.2 \%$. 
CHAPTER 5. NUMERICAL RESULTS AND DISCUSSION

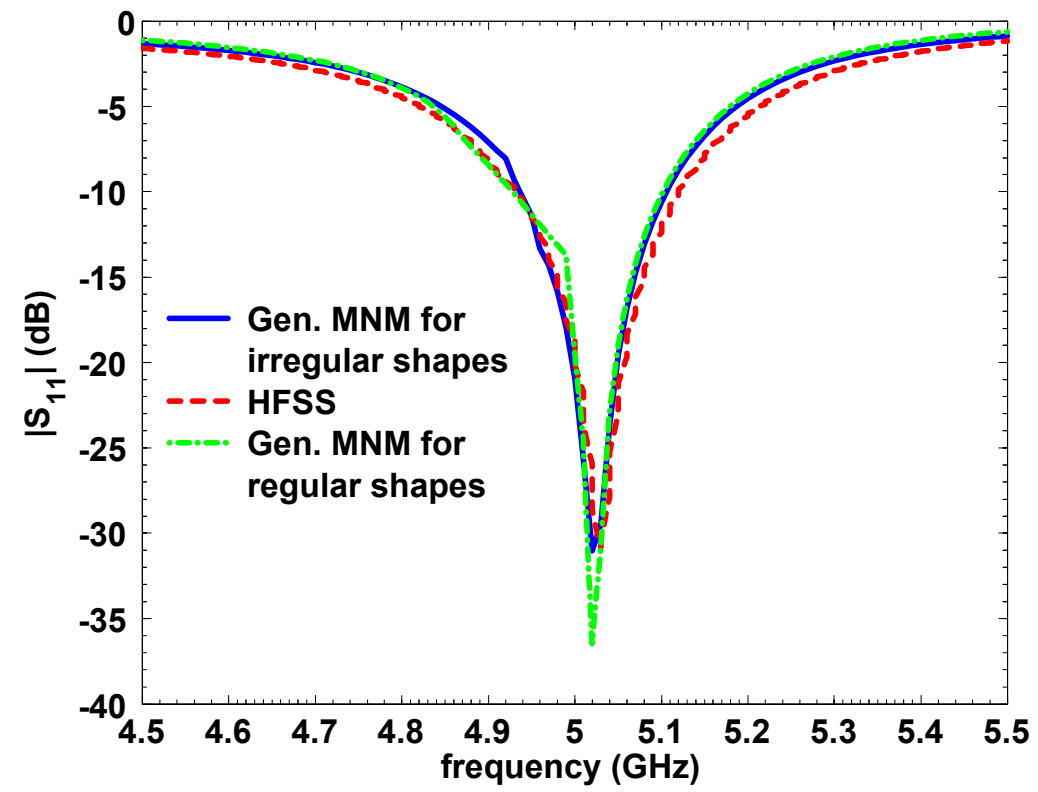

Figure 5.11: $\left|S_{11}\right|$ for Two circular patches coupled through a slot in ground plane

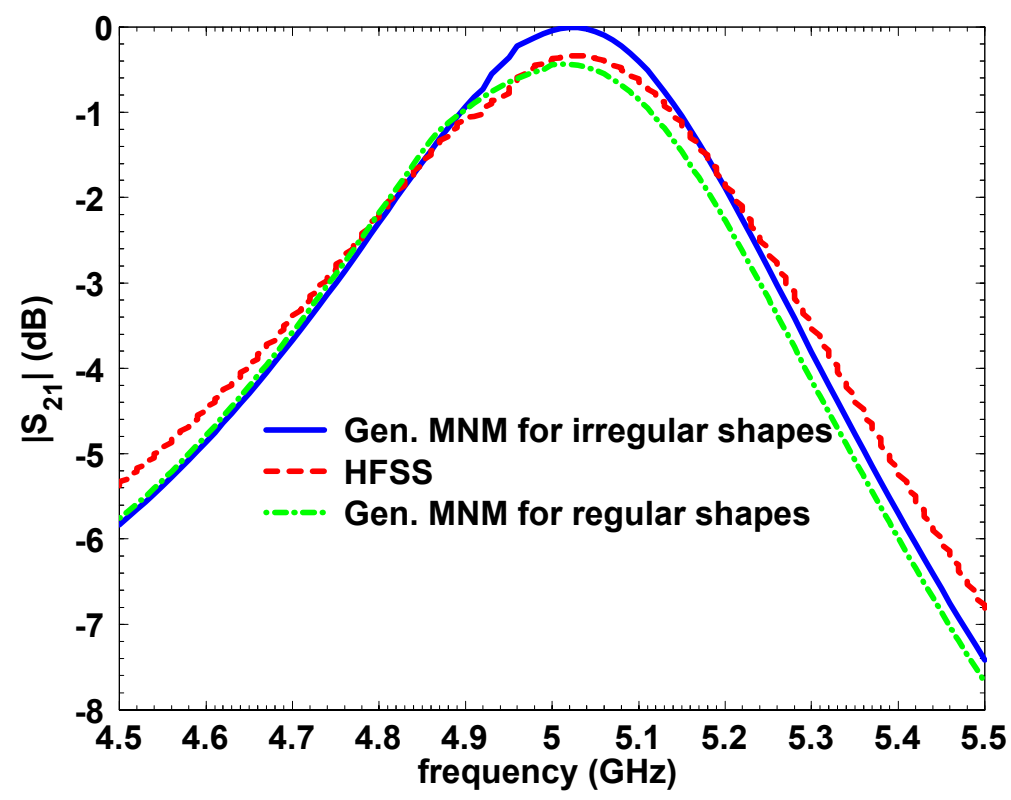

Figure 5.12: $\left|S_{21}\right|$ for Two circular patches coupled through a slot in ground plane 


\subsubsection{Corner-Cut Patches Coupled by an Inclined Slot}

And finally, we consider two corner-cut patches, similar to the ones considered before, but coupled through a slot inclined at an angle of $\varphi=45^{\circ}$ with respect to the $x$-axis, as shown in Fig. 5.13. $\left|S_{11}\right|$ and $\left|S_{21}\right|$ for this structure are compared in Fig. 5.14 and Fig. 5.15, respectively.

The first resonance occurs at $4.93 \mathrm{GHz}$ from our method versus $4.95 \mathrm{GHz}$ from HFSS, being $0.4 \%$ in error. HFSS also predicts a weak second resonance at $5.16 \mathrm{GHz}$, which is absent in our method. Besides, there is a strong transmission zero at $5.17 \mathrm{GHz}$ from our method which is almost absent in HFSS curve. The second resonance can be attributed to radiation. This can be shown by plotting $\sqrt{\left|S_{11}\right|^{2}+\left|S_{21}\right|^{2}}$ as an indication of radiated power. Some power is also launched into surface waves, but due to the fact that the substrates are thin, this can be neglected. Since no loss is considered in generalized MNM, the curve corresponding to the generalized MNM stays at $0 d B$ level for the whole frequency span of interest, except for negligible errors in close vicinity of the resonance frequency (due to numerical errors). Though this structure cannot be considered a good radiator, it still radiates some power. This is specially noticeable at $4.96 \mathrm{GHz}$ (very close to the first resonance) and $5.17 \mathrm{GHz}$ (same as the transmission zero observed in generalized MNM). This explains the discrepancy between the two methods.

In order to further support this claim, the same structure is enclosed in a $72 \mathrm{~mm} \times 72 \mathrm{~mm} \times 24 \mathrm{~mm}$ shielding box and simulated by HFSS. The returnloss of the shielded structure is compared with that of generalized MNM in Fig. 5.17. As can be seen, the second resonance is suppressed. There is a spurious resonance at $4.57 \mathrm{GHz}$, which is very close to the resonance frequencies of the metallic box ( $4.59 \mathrm{GHz}$ for $L S M_{211}^{z}$ and $4.66 \mathrm{GHz}$ for $L S E_{211}^{z}$ ). These resonance and the others in vicinity of $5.8 \mathrm{GHz}$ can be suppressed by increasing the size of the shielding box. But this does not seem to be practical as the shielding boxes are very computationally intensive for HFSS. It can also be seen that by enclosing the structure in a shielding box, resonance frequency from HFSS becomes the same as the resonance frequency from our method. 


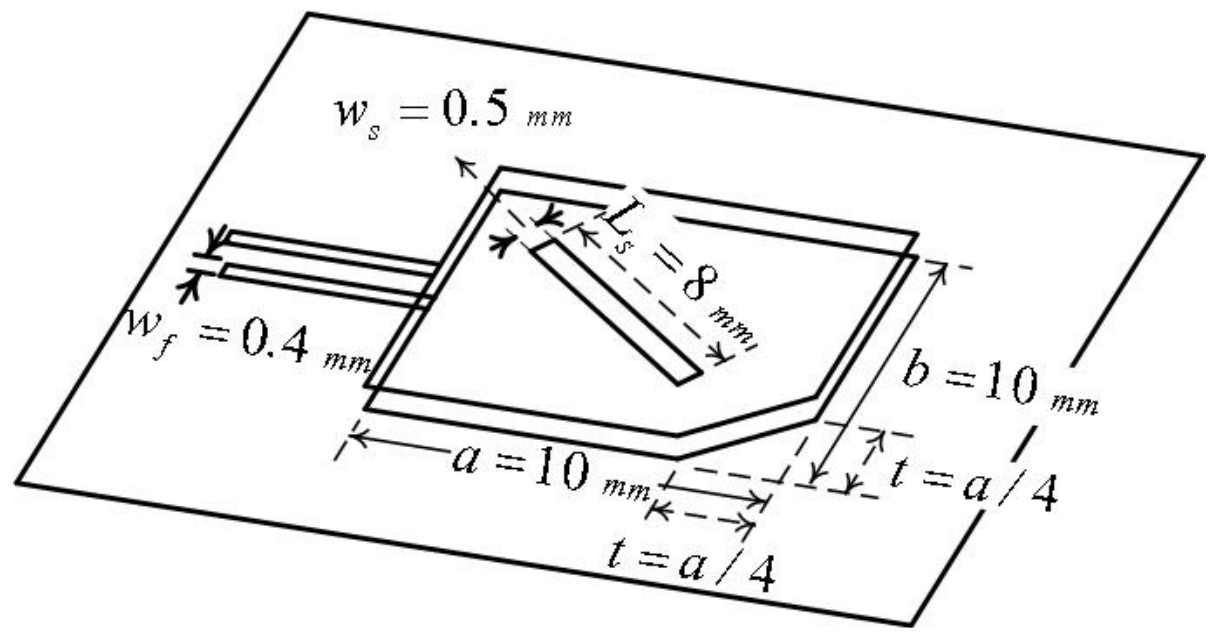

Figure 5.13: Corner-cut patches coupled through an inclined slot

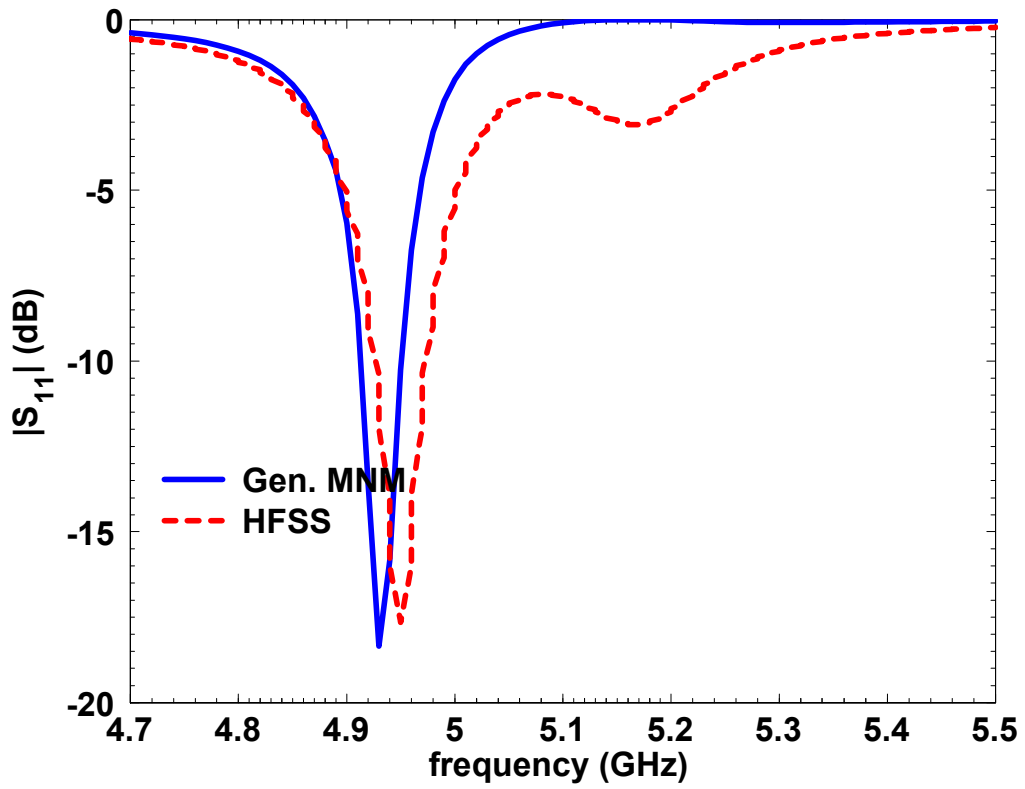

Figure 5.14: $\left|S_{11}\right|$ for corner-cut patches coupled through an inclined slot 


\section{CHAPTER 5. NUMERICAL RESULTS AND DISCUSSION}

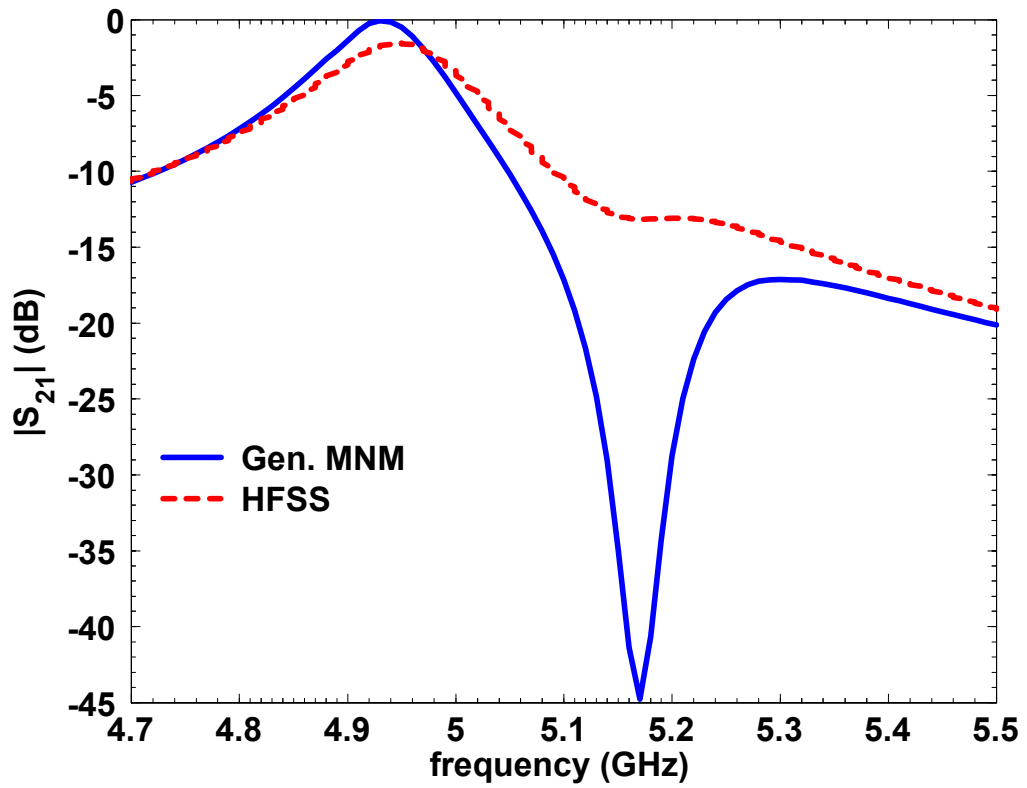

Figure 5.15: $\left|S_{21}\right|$ for corner-cut patches coupled through an inclined slot

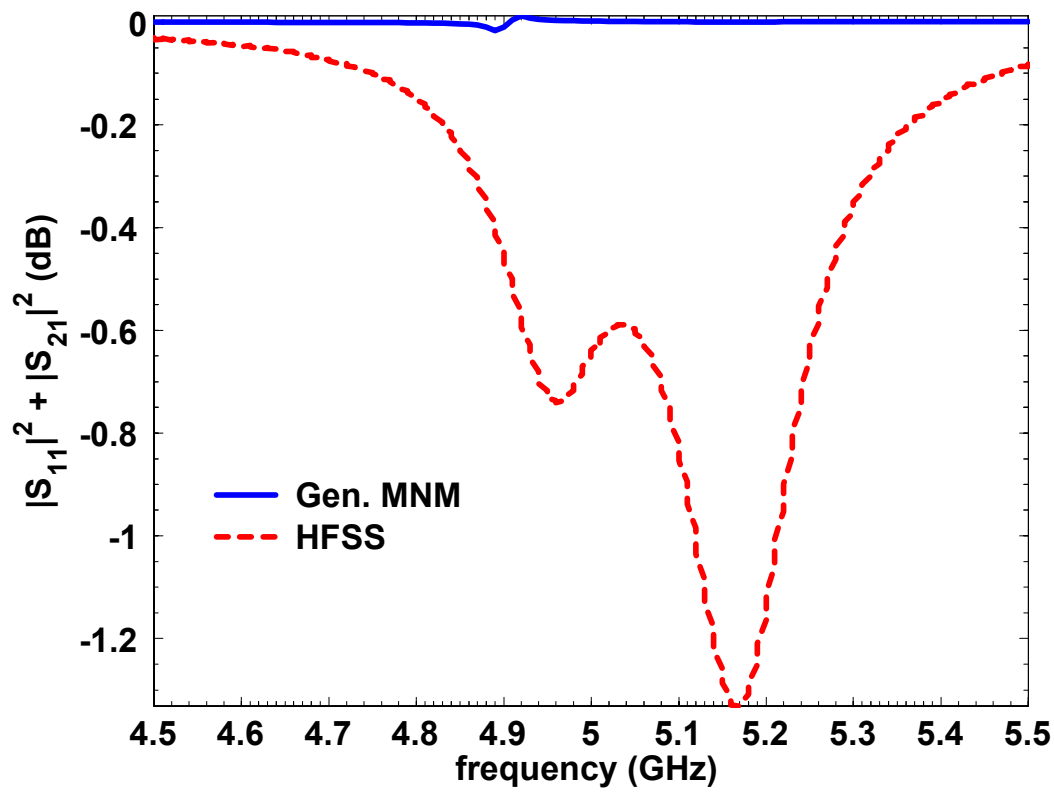

Figure 5.16: Normalized radiated power from structure shown in Fig. 5.13 
CHAPTER 5. NUMERICAL RESULTS AND DISCUSSION

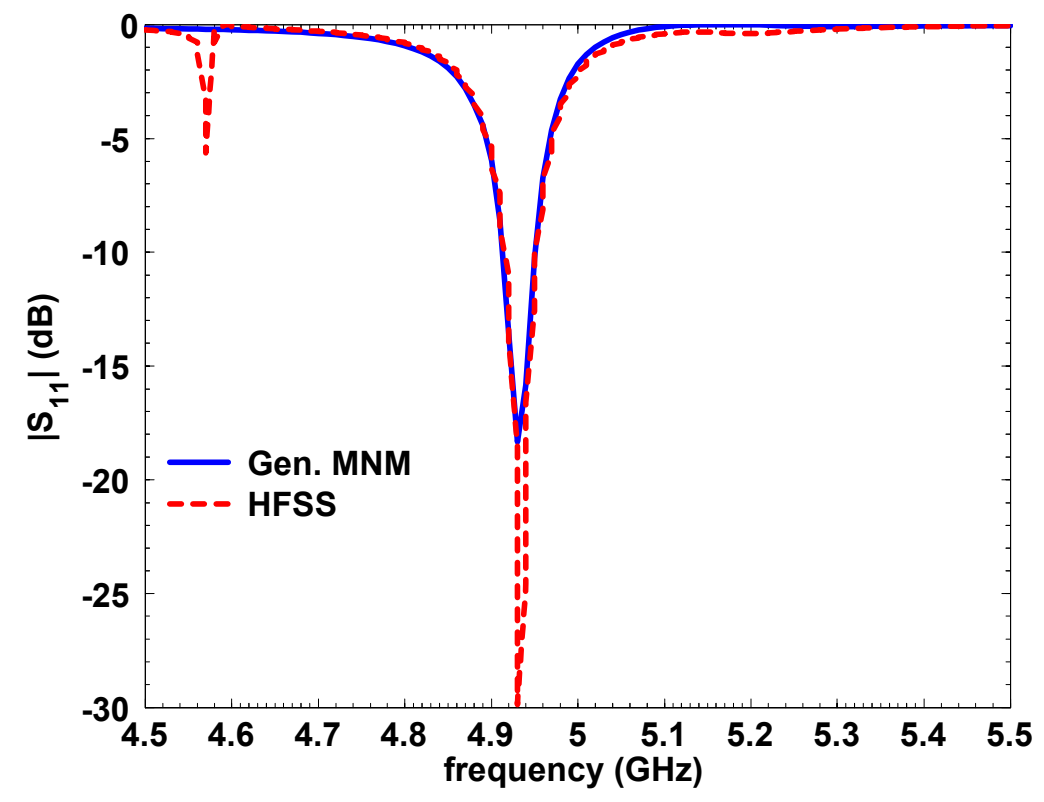

Figure 5.17: $\left|S_{11}\right|$ for the shielded structure in Fig.. 5.13 


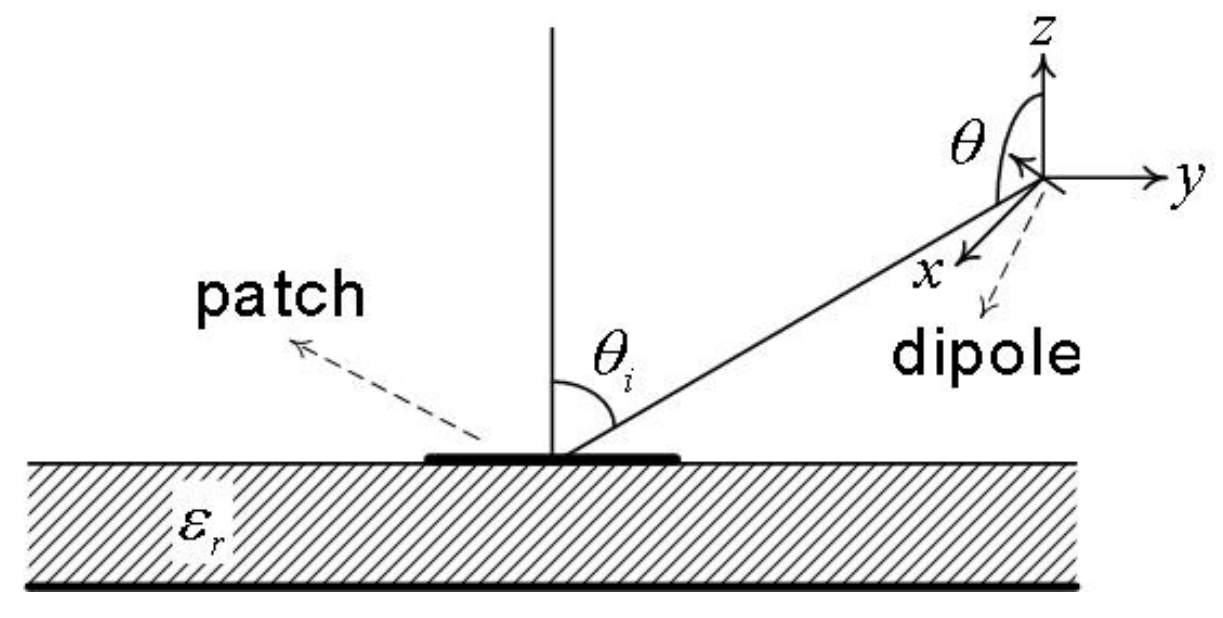

Figure 5.18: Dipole in the far-field of a microstrip antenna

\subsection{Radiation Pattern}

\subsubsection{Reciprocity Approach to Pattern Computation of Microstrip Antennas}

Far-field of a microstrip antenna of an arbitrary shape can be calculated by using reciprocity theorem [103]. The approach presented here is quite similar, except for the fact that instead of surface electric current distribution on the microstrip patch, we have used the reciprocity between the patch and the edge to replace the electric current with the magnetic current on the periphery of circuit pattern [104].

We consider an electric dipole located in the far-field of the microstrip antenna (Fig. 5.18). The magnetic field due to this electric dipole in the presence of the dielectric slab will consist of the incident and reflected fields. Since the dipole is in the far-field of the microstrip patch, the incident field will be a spherical plane wave and hence plane-wave reflection coefficients, as defined in 4.142, can be used to account for the presence of the dielectric slab as follows:

$$
\begin{aligned}
\vec{H}^{\hat{\theta}} & =-j k_{0} I d \ell \frac{e^{-j k_{0} r}}{4 \pi r}\left(1+R^{T M}\right) \hat{\varphi} \\
\vec{H}^{\hat{\varphi}} & =j k_{0} I d \ell \frac{e^{-j k_{0} r}}{4 \pi r}\left[\hat{\rho}\left(1-R^{T E}\right) \cos \theta-\hat{z}\left(1+R^{T E}\right) \sin \theta\right]
\end{aligned}
$$




\section{CHAPTER 5. NUMERICAL RESULTS AND DISCUSSION}

where $\vec{H}^{\hat{\theta}}$ and $\overrightarrow{H^{\varphi}}$ are the fields due to the dipole oriented in $\hat{\theta}$ and $\hat{\varphi}$ directions, respectively. The electric field radiated by a magnetic current distribution on the substrate in the far-field (location of the dipole) can be obtained from reciprocity as:

$$
\begin{aligned}
& \vec{E}_{\theta}=-\frac{1}{I d \ell} \int_{C} \vec{H}^{\hat{\theta}} \cdot \vec{M}^{e d g e} d s \\
& \vec{E}_{\varphi}=-\frac{1}{I d \ell} \int_{C} \vec{H}^{\hat{\varphi}} \cdot \vec{M}^{e d g e} d s
\end{aligned}
$$

where $\vec{E}_{\theta}$ and $\vec{E}_{\varphi}$ are the $\hat{\theta}$ and $\hat{\varphi}$ components of the radiated electric field and $\vec{M}^{\text {edge }}$ is the magnetic current distribution along the edge of the patch. $C$ designates the periphery of the patch.

\subsubsection{Radiation Pattern of an Integrated Antenna-Filter Device}

The electric field pattern for the structure in Fig. 5.1, both from our method and HFSS is shown in Fig. 5.19 and Fig. 5.20. The frequency is chosen to be the resonance frequency of the structure. As can be seen, the agreement is very good for $E_{\varphi}$ all the way up to the grazing angle, but deteriorates for $E_{\theta}$ as we get closer to the grazing angle. This is mainly due to the nature of the method we have used to calculate the pattern. At grazing angle, surface-waves, which have been ignored in the approach based on reciprocity, are dominant. 


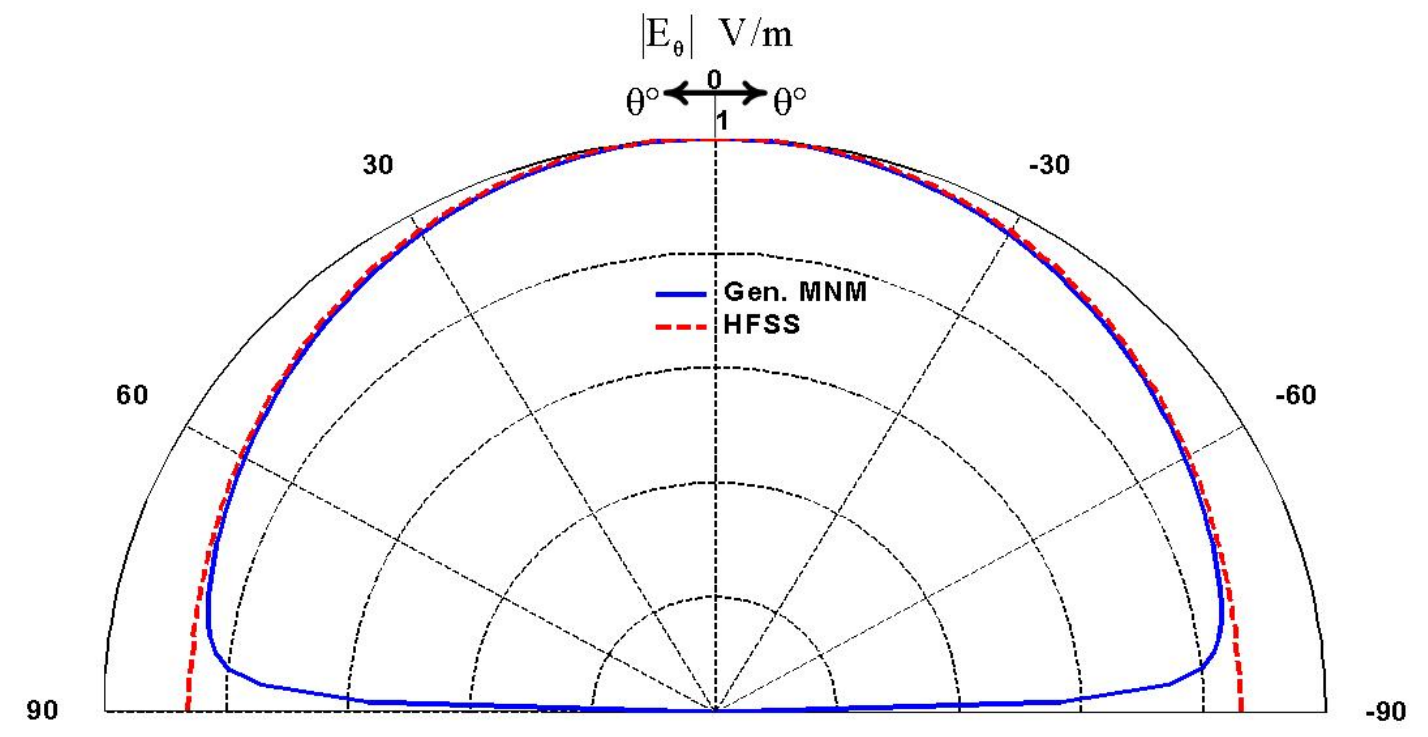

Figure 5.19: $\left|E_{\theta}\right|$ for the structure in Fig. 5.1 at $\varphi=45^{\circ}$

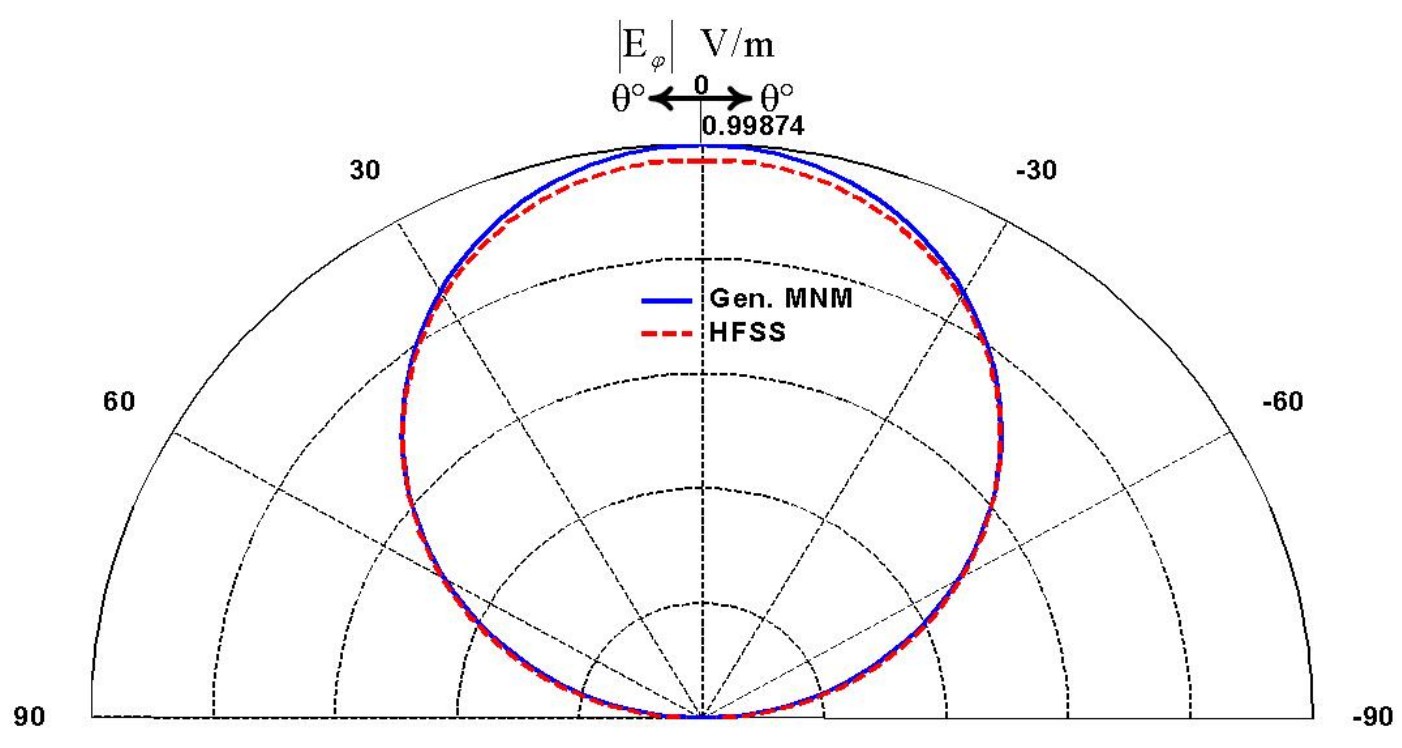

Figure 5.20: $\left|E_{\varphi}\right|$ for the structure in Fig. 5.1 at $\varphi=45^{\circ}$ 


\subsubsection{Radiation Pattern of Two Corner-Cut Patches Coupled by an Inclined Slot}

And finally we present the electric field pattern for the structure in Fig. 5.13, from both our method and HFSS. The results are shown in Fig. 5.21 and Fig. 5.22. The frequency is chosen to be the resonance frequency of the structure. Similar to the previous case, the agreement is very good for $E_{\varphi}$ all the way up to the grazing angle, but deteriorates for $E_{\theta}$ as we get closer to the grazing angle. It can also be observed that in both cases there is better agreement for $E_{\varphi}$ component than for $E_{\theta}$. It can be seen that, particularly in this case, $E_{\theta}$ calculated by reciprocity method starts differing from HFSS at angles very close to the boresight direction, though the difference is not as pronounced as it is at grazing angle. We observed the same problem in other examples as well. This indicates that the reciprocity approach for pattern calculation has to be modified to account for the presence of surface waves along the interface. In addition, in order to improve the far-field pattern from generalized MNM, an iterative approach similar to what is used in conventional MNM can be employed. In iterative approach, a primary edge admittance network is used to calculate the magnetic current on the periphery, which in turn can be used to calculate a new edge admittance matrix [37], [30]. The process continues till it converges. Alternatively, radiation can be added to the generalized MNM by using 2D/3D method in which a correction term is added to the impedance matrix elements [105]. This is part of the future work for this research. 


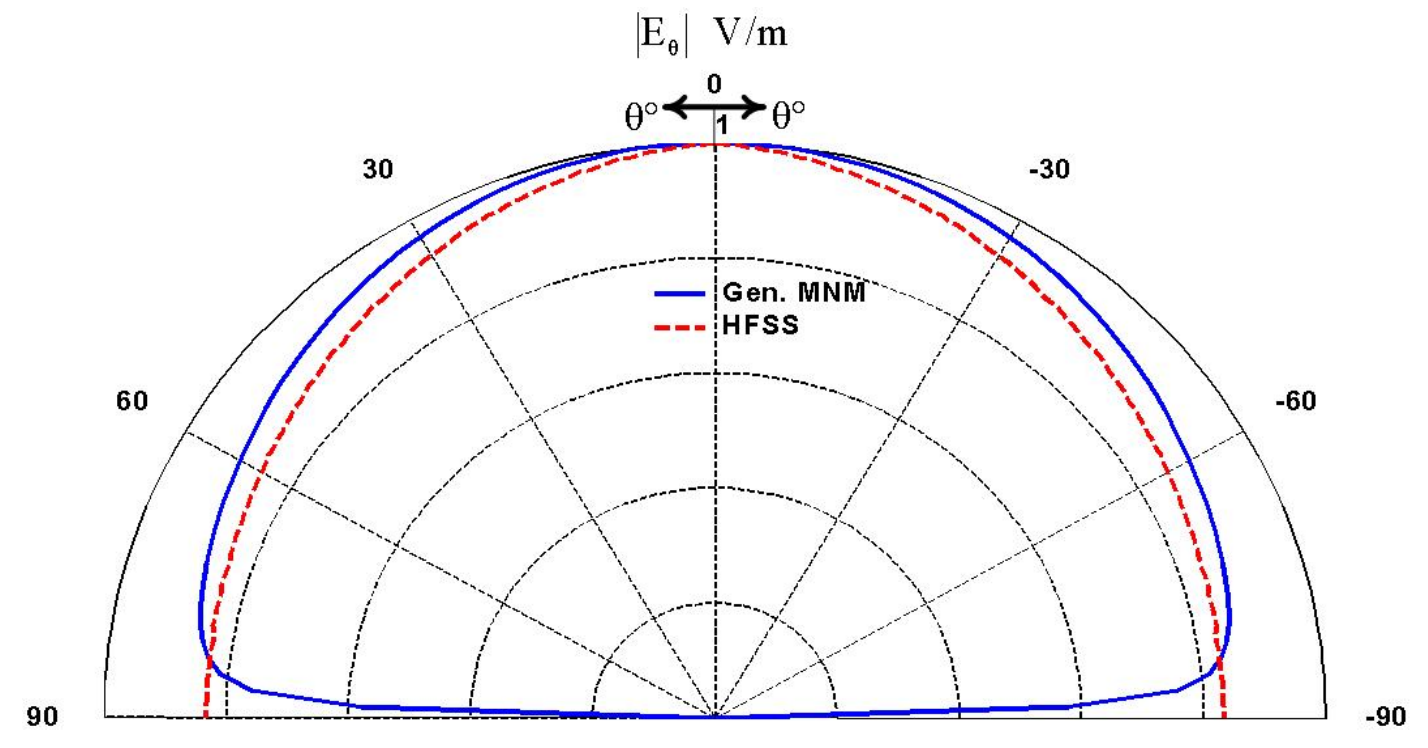

Figure 5.21: $\left|E_{\theta}\right|$ for the structure in Fig. 5.13 at $\varphi=45^{\circ}$

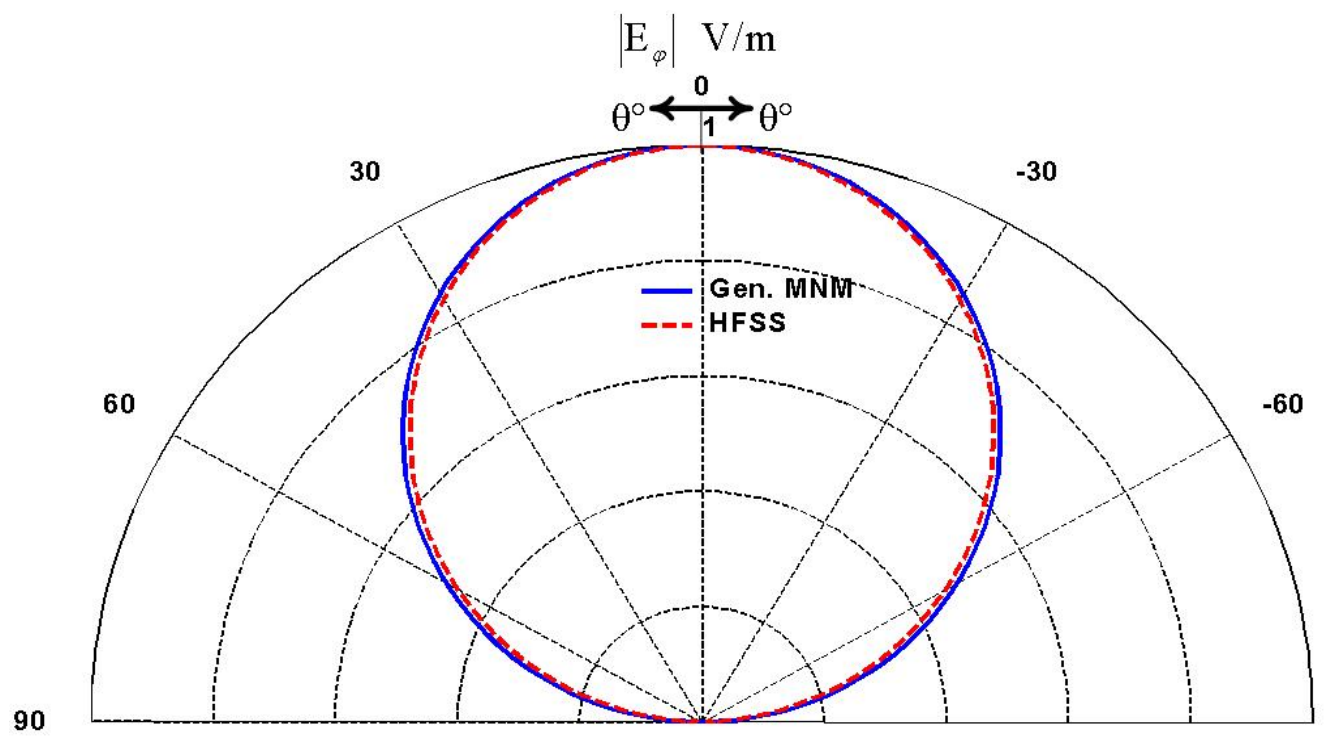

$-90$

Figure 5.22: $\left|E_{\varphi}\right|$ for the structure in Fig. 5.13 at $\varphi=45^{\circ}$ 


\section{Chapter 6}

\section{Conclusion and Future Work}

\subsection{Conclusion}

A new method for fast analysis and design of multi-layer structures consisting of planar circuits coupled through slots in their ground planes has been developed. The proposed method combines generalized network formulation for aperture problems with multiport network model (MNM) for single-layer planar circuits and derives an impedance matrix for the multi-layer structure. We start by replacing the slot with an equivalent magnetic current. In the absence of the slot (or the equivalent magnetic current over the slot), conventional MNM uses a generalized impedance matrix to account for interaction between terminal voltages and currents of edge ports defined along the periphery of circuit pattern. To include the slot in network model, slot ports with terminal voltages and currents need to be defined. In order to avoid imposing any restriction on the shape of the slot, however, we do not define any physical ports over the slot. Instead, the equivalent magnetic current over the slot is expanded in a set of basis functions and the coefficients of expansion are treated as slot port voltages. Similar to generalized network formulation for aperture problems, slot port currents are defined as transverse magnetic field over the slot tested by the basis function corresponding to the slot port voltage. Then we use transfer matrices to account for interaction between edge and slot port voltages as well as interaction between edge and slot port currents. The interaction between slot port voltages and currents is described by a generalized admittance matrix. Therefore, from network model point of view, the structure is seen as a multiport network consisting of edge and slot ports fully characterized by a hybrid matrix. 


\section{CHAPTER 6. CONCLUSION AND FUTURE WORK}

The hybrid matrices of the planar circuit above and below the ground plane are then connected by enforcing network equivalent of electromagnetic boundary conditions, from which an impedance matrix can be derived that accounts for interaction between terminal voltages and currents of both of the planar circuits on either side of the ground plane. Though the slot has been accounted for in the formulation of the problem, it is invisible to the network model.

The proposed approach to network characterization of a multi-layer planar circuit has been implemented by modal analysis for regular shapes and contour integral equation for irregular shapes. To this end, a new contour integral equation has been derived which gives transverse magnetic field inside a closed contour in terms of tangential components of electric and magnetic field on the boundary. When the observation point is on the boundary, we have shown that the new contour integral equation is the inverse of the traditional contour integral equation. Hence, as a side benefit, in conventional MNM an admittance matrix can be directly calculated for a single-layer planar circuit, obviating the need for inverting ill-conditioned matrices.

The new method can be easily extended to more than two layers. Therefore, similar to single-layer circuits in conventional MNM, a multi-layer structure is fully characterized by an impedance matrix in the new method. Hence our method has all the benefits of conventional MNM. Regarding the fact that low-frequency network theory is a very well established discipline and extensive CAD exists for analysis and design optimization of low-frequency circuits, planar microwave circuits can benefit from all available tools through MNM based methods.

The proposed method is also very promising in terms of the future work and what can be done to improve the performance and extend applicability of this method. This will be discussed in the next section.

\section{Limitations of the Proposed Approach}

Like any other approximate numerical method, the proposed approach has its own limitations. Some of these limitations are inherited from traditional MNM for single-layer circuits. One of the fundamental assumptions in MNM for single-layer circuits, which hereinafter will be refered to as traditional MNM, is that the planar circuit is so thin that the cavity can be essentially considered to support only $2 \mathrm{D}$ modes with no variation in longitudinal direction (direction perpendicular to the ground plane). In other words, $d$, the thickness of the dielectric substrate must be much smaller than the transverse dimensions of the metallic patch as well as $\lambda$, 


\section{CHAPTER 6. CONCLUSION AND FUTURE WORK}

the wavelength in the dielectric medium, i.e. $d \ll a$ and $d \ll \lambda$, where $a$ is the transverse dimension of the patch (side of a rectangular patche or radius of a circular patch). As a result, the electric field has no transverse components, meaning that a unique voltage can be defined bewteen the metallic patch and the ground plane. In addition, the boundary of the planar circuit is assumed to be covered by PMC walls, which means that the surface current is normal to the boundary of the patch wherever there is a coupling port and is otherwise zero. This in turn enables us to define input currents at coupling ports.

As $d$ increases, the accuracy of traditional MNM deteriorates. In addition to $T M^{z}$ modes which already exist in a thin planar circuit, $T E^{z}$ modes will also be excited. Consequently, the voltage between the patch and the ground plane is not unique anymore. Furthermore, it can no longer be assumed the planar circuit is surrounded by PMC walls, which in turn invalidates modal expansion for regular shapes and contour integral method for irregular shapes (contour integral equation is independant from this assumption, it is the method based on this equation that fails).

In addition to the aforementioned limitations inherent to MNM for planar circuits, the generalized MNM is also sensitive to the closeness of the slot to the boundary. In deriving the generalized MNM it has been assumed that away from the slot (in particular on the boundary) the fields are essentially $T M^{z}$. However, if the slot is too close to the boundary, this assumption does not hold anymore. Other than the fact that the slot should not be too close to the boundary, there is no restriction on the size or shape or even location of the slot. In most practical applications, however, slots are used to couple energy between different layers without fundamentally disturbing the basic function of the planar structures. Therefore, in practice slots are never too close to the boundary and the generalized MNM can be used to analyze these structures.

\subsection{Future Work}

As it was previously mentioned, the future work on the new method can move in several directions:

1. The proposed approach ignores losses, including radiation losses. Radiation losses can be taken into account either by an Edge Admittance Network 


\section{CHAPTER 6. CONCLUSION AND FUTURE WORK}

(EAN) and consequent application of segmentation method of analysis or by adding a correction term to the impedance matrix elements which accounts for the radiated power (the 2D/3D method) [105]. The latter has the benefit that it does not require matrix inversion and due to the fact that the correction term is derived from a variational expression, rather large relative errors can be tolerated. In addition, we have already developed computer programs for calculating the fields of an HMD and an HED in a planarly layered media using complex images method, hence the aforementioned 2D/3D method can be readily combined with the proposed approach.

2. Our method can be extended to more than two-layers. By taking the radiation losses into account, this method can be used for analysis and optimized design of multi-layer integrated antenna-filter devices. Gap coupling between the planar circuit on the same layer has already been successfully modeled by other authors and can be combined by our method to design highly-complex microwave filters and circuits.

3. A reciprocity based method for analysis of printed slot and slot-coupled microstrip antennas has been described in [4]. The proposed method is capable of extending applicability of the reciprocity-based method to cavity-backed slots, as the slot admittance matrix of a slot radiating into a planar cavity of arbitrary shape can be readily calculated by our method.

4. In cases where the dielectric substrate of planar circuits is planarly layered, our method can be modified to conveniently model such structures. This extends the applicability of our method even further. The vision for such an extension has been one of the main reasons for using complex images method for calculating the fields due to an HMD in a parallel-plate waveguide. 


\section{Appendix A}

\section{Proof of 3.115}

A more general and elaborate proof is given in [51]. The proof given here is very similar, but simpler and limited in scope. We start by using the following identity:

$$
\nabla \phi_{m} \cdot \nabla \phi_{n}^{*}=\nabla \cdot\left(\phi_{m} \nabla \phi_{n}^{*}\right)-\phi_{m} \nabla^{2} \phi_{n}^{*}
$$

to show that:

$$
\begin{aligned}
\iint_{D} \nabla \phi_{m} \cdot \nabla \phi_{n}^{*} d S & =\oint_{C} \phi_{m} \frac{\partial \phi_{n}^{*}}{\partial n} d s-\iint_{D} \phi_{m} \nabla^{2} \phi_{n}^{*} d S \\
& =\oint_{C} \phi_{m} \frac{\partial \phi_{n}^{*}}{\partial n} d s+\left(k^{2}-\beta_{n}^{2}\right) \iint_{D} \phi_{m} \phi_{n}^{*} d S
\end{aligned}
$$

where use has been made of the fact that eigenfunctions satisfy homogeneous wave equation. $C$ is the boundary of region $D$ and $n$ is the outward normal to $C$. Since either $\phi_{m}$ or its normal derivative vanish on the boundary, the first integral vanishes. The second integral is also zero due to orthogonality of eigenfunctions. Therefore:

$$
\iint_{D} \nabla \phi_{m} \cdot \nabla \phi_{n}^{*} d S=0
$$

which proves 3.115 . 


\section{Appendix B}

\section{Derivation of 2.56}

Equation 2.54 gives the potential at any point $P$ inside the contour $C$ in terms of the potential $V(s)$, and its normal derivative $\partial V / \partial s$ on the boundary, where $s$ is the distance measured along the contour $C$ in counterclockwise direction. Suppose that the point $P^{\prime}$ is a point on the contour $C$ and the point $P$ is just inside $C$ in a way that $\delta \ll \alpha \ll \lambda$, as shown in Fig. B.1 [36]. The contour integral in 2.54 can be broken down into two parts, one accounting for the contribution of the part of $C$ between $s=-\alpha$ and $s=\alpha$ and the other accounting for the contribution from the rest of $C$ denoted by $\Gamma$. Therefore one can write [36]:

$$
\begin{aligned}
4 j V(P)= & \frac{2 j}{\pi} \int_{-\alpha}^{\alpha}\left(\frac{\delta}{s^{2}+\delta^{2}} V-\ell n \frac{k \sqrt{s^{2}+\delta^{2}}}{2} \frac{\partial V}{\partial n}\right) d s+ \\
& \int_{\Gamma}\left(H_{0}^{(2)}(k \rho) \frac{\partial V}{\partial n}-\frac{\partial H_{0}^{(2)}(k \rho)}{\partial n} V\right) d s
\end{aligned}
$$

where the following small argument approximations have been used to substitute for $H_{0}^{(2)}(k \rho)$ and $\partial H_{0}^{(2)}(k \rho) / \partial n$ for $s$ between $-\alpha$ and $\alpha$ :

$$
\begin{gathered}
H_{0}^{(2)}(k \rho) \approx-\frac{2 j}{\pi} \ln \frac{k \sqrt{s^{2}+\delta^{2}}}{2} \\
\frac{\partial H_{0}^{(2)}(k \rho)}{\partial n} \approx-\frac{2 j}{\pi} \frac{\delta}{s^{2}+\delta^{2}}
\end{gathered}
$$

Since $\alpha \ll \lambda$, it can be assumed that $V$ and $\partial V / \partial n$ do not change appreciably in the region from $s=-\alpha$ to $s=\alpha$. In that case, the integrals for small argument 


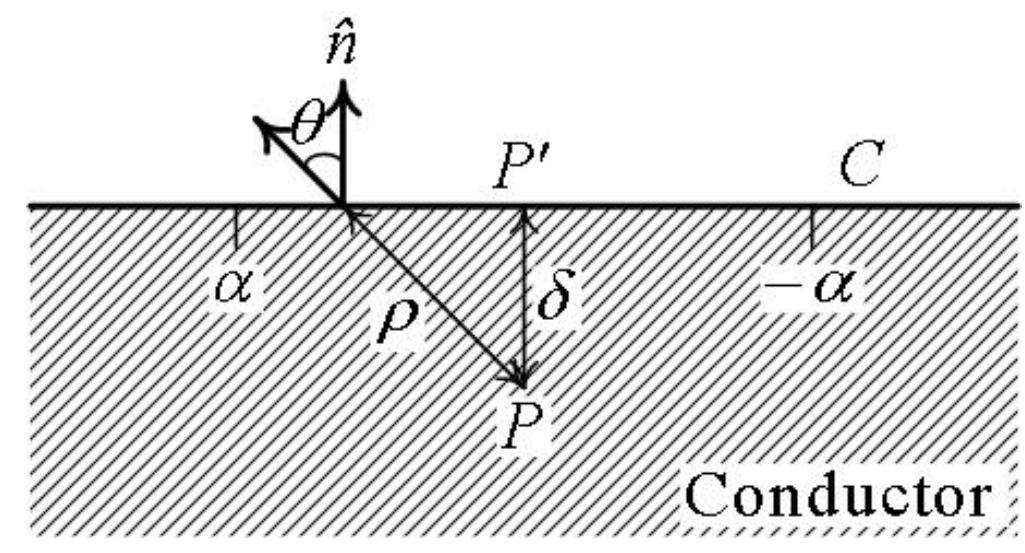

Figure B.1: Symbols used in the derivation of the contour-integral equation [36].

approximations can be evaluated analytically as follows:

$$
\begin{gathered}
I_{1}=\int_{-\alpha}^{\alpha} \frac{\delta}{s^{2}+\delta^{2}} d s=2 \arctan \frac{\alpha}{\delta} \\
I_{2}=\int_{-\alpha}^{\alpha} \ln \frac{k \sqrt{s^{2}+\delta^{2}}}{2} d s=2\left[s\left(\ln \frac{k \sqrt{s^{2}+\delta^{2}}}{2}-1\right)-\delta \arcsin \frac{\delta}{\sqrt{s^{2}+\delta^{2}}}\right]_{0}^{\alpha} \\
=2 \alpha\left(\ln \frac{k \sqrt{\alpha^{2}+\delta^{2}}}{2}-1\right)-2 \delta\left(\arcsin \frac{\delta}{\sqrt{\alpha^{2}+\delta^{2}}}-\frac{\pi}{2}\right)
\end{gathered}
$$

If $\delta$ starts tending towards 0 , the point $P$ will start moving towards the point $P^{\prime}$. In the limit $I_{1}$ and $I_{2}$ become:

$$
\lim _{\delta \rightarrow 0} I_{1}=\pi \quad, \quad \lim _{\delta \rightarrow 0} I_{2}=2 \alpha\left(\ln \frac{k \alpha}{2}-1\right)
$$

By further assuming that $\alpha \longrightarrow 0, I_{2}$ vanishes, $\Gamma$ becomes $C$, and we will have equation 2.56 


\section{Appendix $\mathrm{C}$}

\section{Analytical Evaluation of Convolution Integrals in MPIE}

We first prove that $\mathbf{Y}_{m n}^{p p}$ only depends on $|m-n|$. We have:

$$
\begin{aligned}
\mathbf{Y}_{m n}^{p p} & =\left\langle\vec{H}_{n}^{p p}, \vec{M}_{m}\right\rangle \\
& =-j \omega\left(\varepsilon_{s} I_{F}+\frac{1}{\omega^{2}} I_{q}\right)
\end{aligned}
$$

where

$$
\begin{aligned}
I_{F}^{x x} & =\iint\left[\iint G_{F}^{x x}\left(u-u_{0}, v-v_{0}\right) M_{n}\left(u_{0}\right) d u_{0} d v_{0}\right] M_{m}(u) d u d v \\
I_{q, m}^{x} & =\iint\left[\frac{\partial}{\partial u} \iint G_{q, m}^{x}\left(u-u_{0}, v-v_{0}\right) \frac{\partial M_{n}\left(u_{0}\right)}{\partial u_{0}} d u_{0} d v_{0}\right] M_{m}(u) d u d v
\end{aligned}
$$

and $M_{m}$ and $M_{n}$ are given by 4.190 . By a change of variables as $x=u-u_{0}$ and $y=v-v_{0}$, we will have:

$$
\begin{aligned}
I_{F}^{x x} & =\iint\left[\iint M_{m}(u) M_{n}(u-x) d u d v\right] G_{F}^{x x}(x, y) d x d y \\
I_{q, m}^{x} & =\iint\left[\iint \frac{\partial M_{m}(u)}{\partial u} \frac{\partial M_{n}(u-x)}{\partial u} d u d v\right] G_{q, m}^{x}(x, y) d x d y
\end{aligned}
$$




\section{APPENDIX C. ANALYTICAL EVALUATION OF...}

which can be further processed to show that:

$$
\begin{aligned}
& I_{F}^{x x}=-\frac{2}{w_{s}^{2}} \int_{u_{m}-u_{n}-2 h}^{u_{m}-u_{n}+2 h w_{s}} g(x) y G_{F}^{x x}(x, y) d y d x+\frac{1}{w_{s}} \int_{u_{m}-u_{n}-2 h-w_{s}}^{u_{m}-u_{n}+2 h} \int_{w_{s}}^{w_{s}} g(x) G_{F}^{x x}(x, y) d y d x \\
& I_{q, m}^{x}=\frac{2}{w_{s}^{2}} \int_{u_{m}-u_{n}-2 h}^{u_{m}-u_{n}+2 h w_{s}} h(x) y G_{q, m}^{x}(x, y) d y d x-\frac{1}{w_{s}} \int_{u_{m}-u_{n}-2 h-w_{s}}^{u_{m}-u_{n}+2 h} \int_{w_{s}}^{w_{s}} h(x) G_{q, m}^{x}(x, y) d y d x
\end{aligned}
$$

where $g(x)=M_{m} * M_{n}, h(x)=M_{m}^{\prime} * M_{n}^{\prime}$ (prime indicates differentiation) and can be easily shown to depend only on $|m-n|$. This proves the claim we previously made that $\mathbf{Y}_{m n}^{p p}$ depends solely on $|m-n|$.

$I_{F}^{x x}$ and $I_{q, m}^{x}$ can be quite computationally-intensive if all integrals are calculated numerically. However, as it was mentioned before, for rooftop basis functions the convolution integrals $g(x)$ and $h(x)$, are polynomials, for which some of the integrals in $I_{F}^{x x}$ and $I_{q, m}^{x}$ can be evaluated analytically or at least be reduced to single integrals. For exponential terms in $G_{F}^{x x}$ and $G_{q, m}^{x}$ this has already been done in [96]. Here, we will present the results for Hankel function terms.

In general, in calculation of the fields or the slot admittance matrix using complex images method, evaluation of integrals of the following form, which correspond to Hankel function terms in spatial vector and scalar potentials, is required:

$$
\begin{aligned}
I_{1}^{h f} & =\iint\left(\xi_{3} x^{3}+\xi_{2} x^{2}+\xi_{1} x+\xi_{0}\right) y H_{0}^{(2)}\left(k_{\rho} \rho\right) d y d x \\
& =\int\left(\xi_{3} x^{3}+\xi_{2} x^{2}+\xi_{1} x+\xi_{0}\right) \rho H_{1}^{(2)}\left(k_{\rho} \rho\right) d x \\
& =\frac{\xi_{3} I_{1,3}^{h f}+\xi_{2} I_{1,2}^{h f}+\xi_{1} I_{1,2}^{h f}+\xi_{0} I_{1,0}^{h f}}{k_{\rho}} \\
I_{0}^{h f} & =\iint\left(\xi_{3} x^{3}+\xi_{2} x^{2}+\xi_{1} x+\xi_{0}\right) H_{0}^{(2)}\left(k_{\rho} \rho\right) d y d x \\
& =\xi_{3} I_{0,3}^{h f}+\xi_{2} I_{0,2}^{h f}+\xi_{1} I_{0,2}^{h f}+\xi_{0} I_{0,0}^{h f}
\end{aligned}
$$

where $\xi_{3} x^{3}+\xi_{2} x^{2}+\xi_{1} x+\xi_{0}$ represents either $g(x)$ or $h(x)$, and we have:

$$
\begin{aligned}
I_{1, n}^{h f} & =\int x^{n} \rho H_{1}^{(2)}\left(k_{\rho} \rho\right) d x \\
I_{0, n}^{h f} & =\iint x^{n} H_{0}^{(2)}\left(k_{\rho} \rho\right) d y d x
\end{aligned}
$$




\section{APPENDIX C. ANALYTICAL EVALUATION OF...}

$I_{1,2}^{h f}$ and $I_{1,0}^{h f}$ need to be evaluated analytically. For $I_{1,3}^{h f}$ and $I_{1,1}^{h f}$ we have:

$$
\begin{aligned}
I_{1,3}^{h f} & =\int x^{3} \rho H_{1}^{(2)}\left(k_{\rho} \rho\right) d x \\
& =\int\left(\rho^{2}-y^{2}\right) \rho^{2} H_{1}^{(2)}\left(k_{\rho} \rho\right) d \rho \\
& =-\frac{1}{k_{\rho}} \rho^{4} H_{0}^{(2)}\left(k_{\rho} \rho\right)+\frac{4}{k_{\rho}^{2}} \rho^{3} H_{1}^{(2)}\left(k_{\rho} \rho\right)-\frac{1}{k_{\rho}^{3}}\left(8+y^{2} k_{\rho}^{2}\right) \rho^{2} H_{2}^{(2)}\left(k_{\rho} \rho\right) \\
I_{1,1}^{h f} & =\int x \rho H_{1}^{(2)}\left(k_{\rho} \rho\right) d x \\
& =\int \rho^{2} H_{1}^{(2)}\left(k_{\rho} \rho\right) d \rho \\
& =\frac{1}{k_{\rho}} \rho^{2} H_{2}^{(2)}\left(k_{\rho} \rho\right)
\end{aligned}
$$

Similarly, $I_{0,2}^{h f}$ and $I_{0,0}^{h f}$ can be neither evaluated analytically nor reduced to a single integral. Despite the fact that analytical evaluation of $I_{0,3}^{h f}$ and $I_{0,1}^{h f}$ is not possible either, it is possible to reduce them into single integrals, which can in turn be evaluated numerically.

$$
\begin{aligned}
I_{0,3}^{h f} & =\iint x^{3} H_{0}^{(2)}\left(k_{\rho} \rho\right) d y d x \\
& =\int d y \int\left(\rho^{2}-y^{2}\right) \rho H_{0}^{(2)}\left(k_{\rho} \rho\right) d \rho \\
& =\frac{1}{k_{\rho}^{2}} \int\left[k_{\rho} \rho^{3} H_{1}^{(2)}\left(k_{\rho} \rho\right)-2 \rho^{2} H_{2}^{(2)}\left(k_{\rho} \rho\right)\right] d y-\frac{1}{k_{\rho}} \int y^{2} \rho H_{1}^{(2)}\left(k_{\rho} \rho\right) d(6 \mathrm{C} .8 \mathrm{a}) \\
I_{0,1}^{h f} & =\iint x H_{0}^{(2)}\left(k_{\rho} \rho\right) d y d x \\
& =\int d y \int \rho H_{0}^{(2)}\left(k_{\rho} \rho\right) d \rho \\
& =\frac{1}{k_{\rho}} \int \rho H_{1}^{(2)}\left(k_{\rho} \rho\right) d y
\end{aligned}
$$




\section{Bibliography}

[1] D.M. Pozar and D.H. Schaubert, "Scan blindness in infinite phased arrays of printed dipoles," IEEE Trans. Antennas Propag., vol. AP-32, no. 6, pp. 602-610, June 1984.

[2] D.M. Pozar, "A microstrip antenna aperture coupled to a microstrip line," Electronics Letters, vol. 21, pp. 49-50, Jan. 1985.

[3] D.M. Pozar, "Microstrip antennas," IEEE Proc., vol. 80, pp. 79-91, Jan. 1992.

[4] D.M. Pozar, "A reciprocity method of analysis for printed slot and slotcoupled microstrip antennas," IEEE Trans. Antennas Propag., vol. AP-34, no. 12, pp. 1439-1446, Dec. 1986.

[5] P.L. Sullivan and D.H. Schaubert, "Analysis of an aperture coupled microstrip antenna," IEEE Trans. Antennas Propag., vol. AP-34, no. 8, pp. 977-984, Aug. 1986.

[6] S. Targonski and D.M. Pozar, "Design of wideband circularly polarized aperture coupled microstrip antennas," IEEE Trans. Antennas Propag., vol. AP41, no. 2, pp. 214-220, Feb. 1993.

[7] A. Adrian and D.H. Schaubert, "Dual aperture-coupled microstrip antenna for dual or circular polarisation," Electronics Letters, vol. 23, no. 23, pp. 1226-1228, Nov. 1987.

[8] C.H. Tsao, Y.M. Hwang, F. Killburg, and F. Dietrich, "Aperture-coupled patch antennas with wide-bandwidth and dual-polarization capabilities," in IEEE AP-S International Symposium Digest, 1988, pp. 936-939.

[9] M. Edimo, A. Sharaiha, and C. Terret, "Optimised feeding of dual polarised broadband aperture-coupled printed antenna," Electronics Letters, vol. 28, no. 19, pp. 1785-1787, Sept. 1992. 


\section{BIBLIOGRAPHY}

[10] M.I. Aksun, S.L. Chuang, and Y.T. Lo, "Theory and experiment of electromagnetically excited microstrip antennas for circular polarization operation," in IEEE AP-S International Symposium Digest, 1989, pp. 1142-1145.

[11] T. Vlasits, E. Korolkiewicz, A. Sambell, and B. Robinson, "Performance of a cross-aperture coupled single feed circularly polarised patch antenna," Electronics Letters, vol. 32, no. 7, pp. 612-613, Mar. 1996.

[12] C. Wu, J. Wang, R. Fralich, and J. Litva, "Analysis of a series-fed aperturecoupled patch array antenna," Microwave and Optical Technology Letters, vol. 4, no. 3, pp. 110-113, Feb. 1991.

[13] M. Edimo, P. Rigoland, and C. Terret, "Wideband dual polarized aperture coupled stacked patch antenna array operating in c-band," Electronics Letters, vol. 30, no. 15, pp. 1196-1197, July 1994.

[14] J.P. Daniel, G. Dubost, C. Terret, J. Citerne, and M. Drissi, "Research on planar antennas and arrays: 'structures rayonnantes'," IEEE Antennas and Propag. Magazine, vol. 35, no. 1, pp. 14-38, Feb. 1993.

[15] J.F. Zurcher, "The SSFIP: A global concept for high-performance broadband planar antennas," Electronics Letters, vol. 24, no. 23, pp. 1433-1435, Nov. 1988.

[16] F. Croq and A. Papiernik, "Large bandwidth aperture coupled microstrip antenna," Electronics Letters, vol. 26, no. 16, pp. 1293-1294, Aug. 1990.

[17] F. Croq and A. Papiernik, "Stacked slot-coupled printed antenna," IEEE Microwave and Guided Wave Letters, vol. 1, no. 10, pp. 288-290, Oct. 1991.

[18] F. Croq and D.M. Pozar, "Millimeter wave design of wide-band aperture coupled stacked microstrip antennas," IEEE Trans. Antennas Propag., vol. AP-39, no. 12, pp. 1770-1776, Dec. 1991.

[19] S.D. Targonski and R.B. Waterhouse, "An aperture coupled stacked patch antenna with 50 in IEEE AP-S International Symposium Digest, 1996, pp. $18-21$.

[20] J.A. Curtis and S.J. Fiedziuszko, "Miniature dual mode microstrip filters," in IEEE MTT-S International Microwave Symposium Digest, 1991, pp. 443446. 


\section{BIBLIOGRAPHY}

[21] H.F. Pues and A.R. Van De Capelle, "An impedance-matching technique for increasing the bandwidth of microstrip antennas," IEEE Trans. Antennas Propag., vol. AP-37, no. 11, pp. 1345-1354, Nov. 1989.

[22] H. An, B. Nauwelaers, and A. Van De Capelle, "A new approach of broadband microstrip antenna design," in IEEE AP-S International Symposium Digest, 1992, pp. 475-478.

[23] J.A. Curtis and S.J. Fiedziuszko, "Multi-layered planar filters based on aperture coupled, dual mode microstrip or stripline resonators," in IEEE MTT-S International Microwave Symposium Digest, 1992, pp. 1203-1206.

[24] T. Le Nadan, J.P. Coupez, S. Toutain, and C. Person, "Integration of an antenna/filter device, using a multi-layer, multi-technology process," in 28th European Microwave Conference, 1998, pp. 672-677.

[25] T. Le Nadan, J.P. Coupez, S. Toutain, and C. Person, "Optimization and miniaturization of a filter/antenna multi-function module using a composite ceramic-foam substrate," in IEEE MTT-S International Microwave Symposium Digest, 1999, pp. 219-222.

[26] A. Abbaspour-Tamijani, J. Rizk, and G. Rebeiz, "Integration of filters and microstrip antennas," in IEEE AP-S International Symposium Digest, 2002, pp. $874-877$.

[27] R.B. Waterhouse, D.M. Kokotoff, and C.R. Birtcher, "Highly efficient multilayered printed antenna suitable for MMICs and OEICs," Microwave and Optical Technology Letters, vol. 20, no. 3, pp. 155-156, Feb. 1999.

[28] J.S. Hong and M.J. Lancaster, Microstrip Filters for RF/Microwave Applications, Wiley, New York, 2001.

[29] R.R. Mansour, "Design of superconductive multiplexers using single-mode and dual-mode filters," IEEE Trans. Microwave Theory Tech., vol. MTT-42, no. 7, pp. 1411-1418, July 1994.

[30] A. Benalla and K.C. Gupta, "Multiport network model and transmission characteristics of two-port rectangular microstrip patch antennas," IEEE Trans. Antennas Propag., vol. AP-36, no. 10, pp. 1337-1342, Oct. 1988.

[31] X. Gao and K. Chang, "Network modeling of an aperture coupling between microstrip line and patch antenna for active array applications," IEEE Trans. Microwave Theory Tech., vol. MTT-36, no. 3, pp. 505-513, Mar. 1988. 


\section{BIBLIOGRAPHY}

[32] M. Himdi, J.P. Daniel, and C. Terret, "Analysis of aperture-coupled microstrip antenna using cavity method," Electronics Letters, vol. 25, no. 6, pp. 391-392, Mar. 1989.

[33] M. Himdi, J.P. Daniel, and C. Terret, "Resonant behaviour of slot coupled microstrip antennas," in Proceedings of International Symposium on Antennas and Propagation, 1989, pp. 217-220.

[34] M. Himdi, J.P. Daniel, and C. Terret, "Transmission line analysis of aperturecoupled microstrip antenna," Electronics Letters, vol. 25, no. 18, pp. 12291230, Aug. 1989.

[35] M. El Yazidi, M. Himdi, and J.P. Daniel, "Transmission line analysis of nonlinear slot coupled microstrip antenna," Electronics Letters, vol. 28, no. 15, pp. 1406-1408, July 1992.

[36] T. Okoshi, Planar Circuits for Microwave and Lightwaves, Springer-Verlag, New York, 1985.

[37] J.R. James and P.S. Hall, Handbook of Microstrip Antennas, IEE Electromagnetic Wave Series 28, London, 1989.

[38] R. Sorrentino, "Planar circuits, waveguide models, and segmentation method," IEEE Trans. Microwave Theory Tech., vol. MTT-33, no. 10, pp. 1057-1066, Oct. 1985.

[39] R. Chadha and K.C. Gupta, "Green's functions for triangular segments in planar microwave circuits," IEEE Trans. Microwave Theory Tech., vol. MTT28, no. 10, pp. 1139-1143, Oct. 1980.

[40] K.C. Gupta, R. Garg, and R. Chadha, Computer-Aided Design of Microwave Circuits, Artech House, U.S.A., 1981.

[41] R.F. Harrington, Time-Harmonic Electromagnetic Fields, Wiley-IEEE Press, New York, 2001.

[42] T. Okoshi and T. Miyoshi, "The planar circuit-an approach to microwave integrated circuitry," IEEE Trans. Microwave Theory Tech., vol. MTT-20, no. 4, pp. 245-252, Apr. 1972.

[43] T. Okoshi and T. Takeuchi, "Analysis of planar circuits by segmentation method," Electronics and Communications in Japan, vol. 58-B, no. 8, pp. $71-79,1975$. 


\section{BIBLIOGRAPHY}

[44] R. Chadha and K.C. Gupta, "Segmentation method using impedance matrix for analysis of planar microwave circuits," IEEE Trans. Microwave Theory Tech., vol. MTT-29, no. 1, pp. 71-74, January 1981.

[45] M.J. Beaubien and A. Wexler, "An accurate finite-difference method for high-order waveguide modes," IEEE Trans. Microwave Theory Tech., vol. MTT-16, no. 12, pp. 1007-1017, Dec. 1968.

[46] P. Silvester, "A general high-order finite-element waveguide analysis program," IEEE Trans. Microwave Theory Tech., vol. MTT-17, no. 4, pp. 204210, Apr. 1969.

[47] R.H.T. Bates, "The theory of the point-matching method for perfectly conducting waveguides and transmission lines," IEEE Trans. Microwave Theory Tech., vol. MTT-17, no. 6, pp. 294-301, June 1969.

[48] R.M. Bulley and J.B. Davis, "Computation of approximate polynomial solutions to TE modes in an arbitrarily shaped waveguide," IEEE Trans. Microwave Theory Tech., vol. MTT-17, no. 8, pp. 440-446, Aug. 1969.

[49] R.M. Bulley, "Analysis of the arbitrarily shaped waveguide by polynomial approximation," IEEE Trans. Microwave Theory Tech., vol. MTT-18, no. 12, pp. 1022-1028, Dec. 1970.

[50] J.A. Stratton, Electromagnetic Theory, McGraw-Hill, New York, 1941.

[51] R.E. Collin, Field Theory of Guided Waves, IEEE-OUP Press, New York, 1991.

[52] N. Amitay, V. Galindo, and C.P. Wu, Theory and Analysis of Phased Array Antennas, Wiley, New York, 1972.

[53] R.F. Harrington and J.R. Mautz, "A generalized network formulation for aperture problems," IEEE Trans. Antennas Propag., vol. AP-24, no. 6, pp. 870-873, November 1976.

[54] R.F. Harrington, Field Computation by Moment Methods, Macmillan, New York, 1968.

[55] A. Khajehnasiri and S. Safavi-Naeini, "Multi-port network modeling of multilayer planar circuits containing ground plane slots," in 9th International Symposium on Antenna Technology and Applied Electromagnetics (ANTEM), Montréal, QC, July 31 - August 22002. 


\section{BIBLIOGRAPHY}

[56] A. Khajehnasiri and S. Safavi-Naeini, "An improved multi-port network model for multi-layer planar circuits," in IEEE AP-S International Symposium Digest, Columbus, Ohio, June 22-27 2003.

[57] J.J. Yang, Y.L. Chow, G.E. Howard, and D.G. Fang, "Complex images of an electric dipole in homogeneous and layered dielectrics between two ground planes," IEEE Trans. Microwave Theory Tech., vol. MTT-40, no. 3, pp. 595-600, March 1992.

[58] K.A. Michalski, "The mixed-potential electric field integral equation for objects in layered media," Arch. Elek. Ubertragung, vol. 39, no. 5, pp. 317-322, September/October 1985.

[59] K.A. Michalski and J.R. Mosig, "Multilayered media greens functions in integral equation formulations," IEEE Trans. Antennas Propag., vol. AP-45, no. 3, pp. 508-519, Mar. 1997.

[60] J.R. Mosig and F.E. Gardiol, A Dynamical Radiation Model for Microstrip Structures, Advances in Electronics and Electron Physics. Academic Press, New York, 1982.

[61] J.R. Mosig and F.E. Gardiol, "A dynamic vector potential theory for threedimensional microstrip structures," Bull. AGEN, vol. 26, pp. 45-52, Nov. 1978.

[62] J.R. Mosig and F.E. Gardiol, "The near field of an open microstrip structure," in IEEE AP-S International Symposium Digest, 1979, pp. 379-382.

[63] J.R. Mosig and F.E. Gardiol, "Analytical and numerical techniques in the green's function treatment of microstrip antennas and scatterers," Inst. Elect. Eng. Proc., vol. 130, pt. H, no. 2, pp. 175-182, Mar. 1983.

[64] W.C. Chew, Waves and Fields in Inhomogeneous Media, Van Nostrand Reinhold, New York, 1990.

[65] J.A. Kong, Theory of Electromagnetic Waves, Wiley, New York, 1975.

[66] J.A. Kong, Electromagnetic Wave Theory, Wiley, New York, 1990.

[67] D.G. Fang, J.J. Yang, and G.Y. Delisle, "Discrete image theory for horizontal electric dipoles in a multilayered medium," IEE Proceedings, vol. 135, pt. H, no. 5, pp. 297-303, Oct. 1988. 


\section{BIBLIOGRAPHY}

[68] Y.L. Chow, J.J. Yang, D.G. Fang, and G.E. Howard, "A closed-form spatial green's function for the thick microstrip substrate," IEEE Trans. Microwave Theory Tech., vol. MTT-39, no. 3, pp. 588-592, Mar. 1991.

[69] P. Silvester, "TEM wave properties of microstrip transmission lines," Proc. IEE, vol. 115, no. 1, pp. 43-48, Jan. 1968.

[70] Y.L. Chow and I.N. El-Behery, "An approximate dynamic spatial green's function for microstriplines," IEEE Trans. Microwave Theory Tech., vol. MTT-26, no. 12, pp. 978-983, Dec. 1978.

[71] Y.L. Chow, "An approximate dynamic green's function in three dimensions for finite length microstripline," IEEE Trans. Microwave Theory Tech., vol. MTT-28, no. 4, pp. 393-397, Apr. 1980.

[72] E. Alanen, I.V. Lindell, and A.T. Hujanen, "Exact image method for field calculation in horizontally layered medium above a conducting ground plane," IEE Proceedings, vol. 133, Pt. H, no. 4, pp. 297-304, Aug. 1986.

[73] I.V. Lindell and E. Alanen, "Exact image theory for the sommerfeld halfspace problem. II. vertic electrical dipole," IEEE Trans. Antennas Propag., vol. AP-32, no. 8, pp. 841-849, Aug. 1984.

[74] I.V. Lindell and E. Alanen, "Exact image theory for the sommerfeld halfspace problem. III. general formulation," IEEE Trans. Antennas Propag., vol. AP-32, no. 10, pp. 1027-1032, Oct. 1984.

[75] I.V. Lindell and E. Alanen, "Exact image theory for the sommerfeld halfspace problem. i. vertical magnetic dipole," IEEE Trans. Antennas Propag., vol. AP-32, no. 2, pp. 126-133, Feb. 1984.

[76] I.V. Lindell, E. Alanen, and H.V. Bagh, "Exact image theory for the calculation of fields transmitted through a planar interface of two media," IEEE Trans. Antennas Propag., vol. AP-34, no. 2, pp. 129-137, Feb. 1986.

[77] M.I. Aksun and R. Mittra, "Derivation of closed form spatial green's functions for printed circuit structures with substrates and superstrates," in IEEE APS International Symposium Digest, 1991, pp. 1356-1359.

[78] M.I. Aksun and R. Mittra, "Derivation of closed-form green's functions for a general microstrip geometry," IEEE Trans. Antennas Propag., vol. MTT-40, no. 11, pp. 2055-2062, Nov. 1992. 


\section{BIBLIOGRAPHY}

[79] N. Hojjat, S. Safavi-Naeini, R. Faraji-Dana, and Y.L. Chow, "Fast computation of the nonsymmetrical components of the green's function for multilayer media using complex images," IEE Proc., Microw. Antennas Propag., vol. 145, no. 4, pp. 285-288, Aug. 1998.

[80] M.I. Aksun and G. Dural, "Closed-form green's functions of HED, HMD, VED, and VMD for multilayer media," in IEEE AP-S International Symposium Digest, 1993, pp. 354-357.

[81] M.I. Aksun and R. Mittra, "Efficient use of closed-form green's functions for three-dimensional problems involving multilayered media," in IEEE AP-S International Symposium Digest, 1994, pp. 1354-1357.

[82] M.I. Aksun, "Efficient and robust approach for the derivation of closed-form green's functions," in IEEE AP-S International Symposium Digest, 1995, pp. 1274-1277.

[83] N. Kinayman and M.I. Aksun, "Comparative study of acceleration techniques for integrals and series in electromagnetic problems," in IEEE AP-S International Symposium Digest, 1995, pp. 1037-1040.

[84] G. Dural and M.I. Aksun, "Closed-form green's functions for general sources and stratified media," IEEE Trans. Microwave Theory Tech., vol. MTT-43, no. 7, pp. 1545-1552, July 1995.

[85] M.I. Aksun, "A robust approach for the derivation of closed-form green's functions," IEEE Trans. Microwave Theory Tech., vol. MTT-44, no. 5, pp. 651-658, May 1996.

[86] R.A. Kipp and C.H. Chan, "Complex image method for sources in bounded regions of multilayer structures," IEEE Trans. Microwave Theory Tech., vol. MTT-42, no. 5, pp. 860-865, May 1994.

[87] M.I. Aksun, E.M. Yavuz, and G. Dural, "Comments on the problems in DCIM," in IEEE AP-S International Symposium Digest, 2003, pp. 673-676.

[88] N. Hojjat, S. Safavi-Naeini, and Y.L. Chow, "Numerical computation of complex image green's functions for multilayer dielectric media: Near-field zone and the interface region," IEE Proc., Microw. Antennas Propag., vol. 145, no. 6, pp. 449-454, Dec. 1998. 


\section{BIBLIOGRAPHY}

[89] Y.L. Chow, N. Hojjat, S. Safavi-Naeini, and R. Faraji-Dana, "Spectral green's functions for multilayer media in a convenient computational form," IEE Proc., Microw. Antennas Propag., vol. 145, no. 1, pp. 85-91, Feb. 1998.

[90] J.R. Mosig, Numerical Techniques for Microwave and Millimeter-Wave Passive Structures, Wiley, New York, 1989.

[91] C.A. Balanis, Antenna Theory: Analysis and Design, Wiley, 2nd edition, 1996.

[92] F.B. Hildebrand, Introduction to Numerical Analysis, McGraw-Hill, 1974.

[93] A. Khajehnasiri and S. Safavi-Naeini, "Complex images for a horizontal magnetic dipole in a thin parallel-plate waveguide," in International Association of Science and Technology for Development (IASTED) Conference on Antennas, Radar, and Wave Propagation (ARP 2005), Banff, Alberta, Canada, 2005.

[94] J. Duncan, The Elements of Complex Analysis, Wiley, New York, 1968.

[95] J.W. Brown and R.V. Churchill, Complex Variables and Applications, McGraw-Hill, New York, 2004.

[96] L. Alatan, M.I. Aksun, K. Mahadevan, and M.T. Birand, "Analytical evaluation of MoM matrix elements," IEEE Trans. Microwave Theory Tech., vol. MTT-44, no. 4, pp. 519-525, Apr. 1996.

[97] M.I. Aksun and R. Mittra, "Estimation of spurious radiation from microstrip etches using closed-form green's functions," IEEE Trans. Microwave Theory Tech., vol. MTT-40, no. 11, pp. 2063-2069, Nov. 1992.

[98] M.I. Aksun and R. Mittra, "Choices of expansion and testing functions for the method of moments applied to a class of electromagnetic problems," IEEE Trans. Microwave Theory Tech., vol. MTT-41, no. 3, pp. 503-509, Mar. 1993.

[99] N. Kinayman, M.I. Aksun, and R. Mittra, "On the evaluation of spatial domain MoM matrix entries containing closed form green's functions," in IEEE AP-S International Symposium Digest, 1997, pp. 2346-2349.

[100] N. Kinayman and M.I. Aksun, "Efficient evaluation of spatial-domain MoM matrix entries in the analysis of planar stratified geometries," IEEE Trans. Microwave Theory Tech., vol. MTT-48, no. 2, pp. 309-312, Feb. 2000. 


\section{BIBLIOGRAPHY}

[101] L. Alatan, M.I. Aksun, and T. Birand, "Improving the numerical efficiency of the method of moments for printed geometries," in IEEE AP-S International Symposium Digest, 1994, pp. 1689-1701.

[102] A. Benalla and K.C. Gupta, "Faster computation of z-matrices for rectangular segments in planar microstrip circuits," IEEE Trans. Microwave Theory Tech., vol. MTT-34, no. 6, pp. 733-736, June 1986.

[103] R. Mittra, S. Dey, S. Chakravarty, and N.V. Veremey, "Reciprocity approach to pattern computation of microstrip antennas operating in a complex environment," in IEEE AP-S International Symposium Digest, 1998, pp. 11381141.

[104] S. Chuang, L. Tsang, J. Kong, and W. Chew, "The equivalence of the electric and magnetic surface current approaches in microstrip antenna studies," IEEE Trans. Antennas Propag., vol. AP-28, no. 4, pp. 569- 571, July 1980.

[105] A. Torabian and Y.L. Chow, "A simple 2-d/3-d method for fast analysis of patch resonators with high accuracy," IEEE Trans. Antennas Propag., vol. AP-49, no. 3, pp. 470-474, Mar. 2001. 